Patrícia Helena Figueirêdo do Vale Capucho

\title{
“Test of Practical Judgment” (TOP-J): adaptação brasileira em amostra de indivíduos cognitivamente saudáveis, com comprometimento cognitivo leve e demência
}

\author{
Dissertação apresentada à Faculdade de Medicina \\ da Universidade de São Paulo para obtenção do \\ título de Mestre em Ciências
}

Programa de Neurologia

Orientadora: Profa. Dra. Sonia Maria Dozzi Brucki

(Versão corrigida. Resolução CoPGr 5890, de 20 de dezembro de 2010.

A versão original está disponível na Biblioteca FMUSP)

São Paulo, 2015 
Patrícia Helena Figueirêdo do Vale Capucho

\title{
“Test of Practical Judgment” (TOP-J): adaptação brasileira em amostra de indivíduos cognitivamente saudáveis, com comprometimento cognitivo leve e demência
}

\author{
Dissertação apresentada à Faculdade de Medicina \\ da Universidade de São Paulo para obtenção do \\ título de Mestre em Ciências
}

Programa de Neurologia

Orientadora: Profa. Dra. Sonia Maria Dozzi Brucki

(Versão corrigida. Resolução CoPGr 5890, de 20 de dezembro de 2010.

A versão original está disponível na Biblioteca FMUSP)

São Paulo, 2015 
Dados Internacionais de Catalogação na Publicação (CIP)

Preparada pela Biblioteca da

Faculdade de Medicina da Universidade de São Paulo

Creprodução autorizada pelo autor

Capucho, Patrícia Helena Figueirêdo do Vale

"Test of Pratical Judgment" (TOP-J) : adaptação brasileira em amostra de

indivíduos cognitivamente saudáveis, comprometimento cognitivo leve e demência / Patrícia Helena Figueirêdo do Vale Capucho. -- São Paulo, 2015.

Dissertação(mestrado)--Faculdade de Medicina da Universidade de São Paulo. Programa de Neurologia

Orientadora: Sonia Maria Dozzi Brucki.

Descritores: 1.Julgamento 2.Comprometimento cognitivo leve 3.Demência 4.Doença de Alzheimer 5.Demência Frontotemporal 6.Testes neuropsicológicos

USP/FM/DBD-185/15 
Dedico esse trabalho ao meu pai, Francisco Vale, pelo incentivo em relação à pesquisa e por ser o meu modelo de pesquisador; À minha mãe, Patrícia, à minha avó, Cecília e à minha irmã, Ludmilla, por estarem ao meu lado, me motivando, sempre; E ao meu marido Allisson, pelo companheirismo e apoio, constantes e essenciais. 


\section{AGRADECIMENTOS}

Agradeço a Deus, pela vida, pela minha família e por todas as oportunidades.

Aos meus pais, Francisco e Patrícia, pelo amor incondicional e pela dedicação, confiança e incentivo. Devo a vocês esta conquista.

À minha amada avó Cecília, pelo apoio em tudo, pelas palavras de carinho, pelo incentivo nos momentos difíceis, pela felicidade diante das minhas vitórias e por existir na minha vida.

Ao meu avô Paulo Zábulon, exemplo de honestidade, caráter, profissionalismo e dedicação à pesquisa.

Aos meus irmãos e em especial, à minha querida irmã, Ludmilla, pela motivação constante, por ser mais que irmã, por ser minha amiga de todas as horas, confidente, companheira e parceira.

À minha tia e madrinha Paula, pelas palavras amigas, pelo amor e acolhimento, sempre.

Ao meu marido, Allisson, meu amor, por ser meu amigo e companheiro, pelo incentivo, pela admiração ao meu trabalho e por toda a compreensão.

Ao professor Dr. Ricardo Nitrini, por todos os ensinamentos e por ser um exemplo de profissional e de líder.

À minha orientadora, professora Dra. Sonia Maria Dozzi Brucki, por ter contribuído com meu aprendizado, de forma tão relevante, pelos questionamentos, pelas respostas e pelo incentivo.

À Dra. Laura Rabin, por ter concedido o TOP-J e permitido que este estudo fosse realizado, pela simpatia, pelas correções, pelos artigos enviados e pela disponibilidade em ajudar. 
À Dra. Maria Teresa Carthery-Goulart pela importante contribuição no exame de qualificação.

À Dra. Márcia Lorena Fagundes Chaves, pela importante participação no exame de qualificação.

À Dra. Mônica Sanches Yassuda pelas valiosas contribuições no exame de qualificação, pela humildade, companheirismo e pelo empenho na transmissão do conhecimento em neuropsicologia.

Aos colegas do Grupo de Neurologia Cognitiva e do Comportamento (GNCC), pelo companheirismo, pela troca de conhecimentos e por toda a ajuda na realização deste trabalho.

À Dra. Cláudia Sellitto Porto, por me receber na equipe de neuropsicologia e pelos ensinamentos transmitidos.

À amiga Lívia Spíndola, querida e especial, por toda a parceria ao longo desses anos e por ter estado presente em todos os momentos.

À Simone Oliveira, secretaria do Centro de Referências em Distúrbios Cognitivos, às voluntárias do ambulatório de neurologia, Dona Mary e Cidinha e à secretária da pós graduação da neurologia, Thaís Figueira, pela disponibilidade em ajudar.

A todos os pacientes, acompanhantes e voluntários que participaram deste estudo, meu sincero respeito e agradecimento.

À FAPESP, Fundação de Amparo à Pesquisa do Estado de São Paulo, pelo auxílio financeiro ao estudo (Processo 2012/15019-6). 
"Se em um dia de tristezas, tiveres que escolher entre o mundo e o amor... escolhas o amor, e com ele conquiste o mundo!"

Albert Einstein (1879-1955) 


\section{NORMALIZAÇÃO ADOTADA}

Esta dissertação ou tese está de acordo com as seguintes normas, em vigor no momento desta publicação:

Referências: adaptado de International Committee of Medical Journals Editors (Vancouver).

Universidade de São Paulo. Faculdade de Medicina. Divisão de Biblioteca e Documentação. Guia de apresentação de dissertações, teses e monografias. Elaborado por Anneliese Carneiro da Cunha, Maria Julia de A. L. Freddi, Maria F. Crestana, Marinalva de Souza Aragão, Suely Campos Cardoso, Valéria Vilhena. 3a ed. São Paulo: Divisão de Biblioteca e Documentação; 2011.

Abreviaturas dos títulos dos periódicos de acordo com List of Journals Indexed in Index Medicus. 


\section{SUMÁRIO}

LISTA DE SIGLAS E ABREVIATURAS

LISTA DE SÍMBOLOS

LISTA DE FIGURAS

LISTA DE TABELAS

RESUMO

ABSTRACT

1. Introdução.....................................................................1

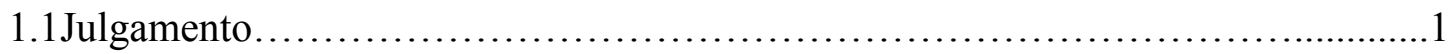

1.2 Demência.................................................................

1.2.1 Doença de Alzheimer...................................................4

1.2.2 Demência Frontotemporal................................................

1.3 Comprometimento Cognitivo Leve.......................................6

1.4 Avaliação de Julgamento................................................. 8

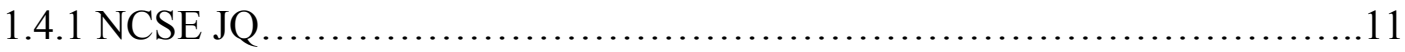

1.4.2 Subescala Resolução de Problemas da ILS...............................12

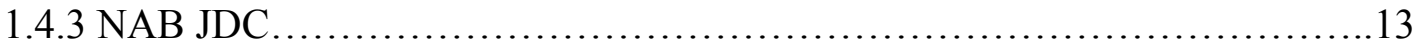

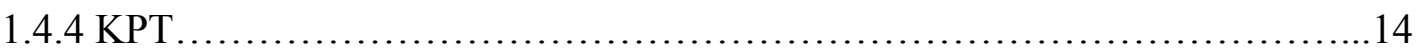

1.4.5 Test of Practical Judgment (TOP-J) ..................................18

1.5 Julgamento em Demência...............................................25

1.6 Julgamento em Comprometimento Cognitivo Leve...........................29

1.7 Justificativa.................................................................

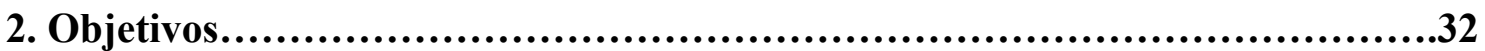

2.1 Objetivo Geral...................................................... 32

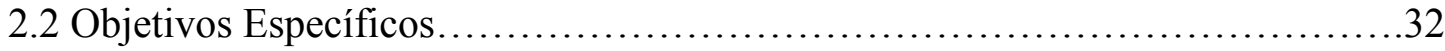




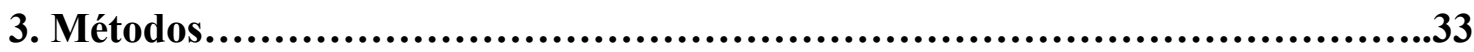

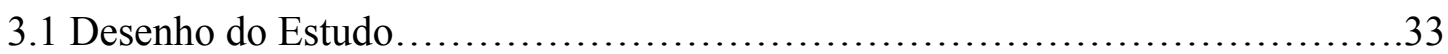

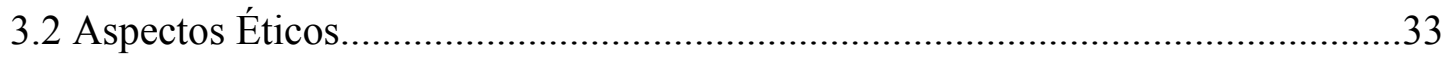

3.3 Casuística.............................................................. 33

3.3.1 Grupo Controle...................................................... 34

3.3.2 Grupo de Pacientes................................................. 35

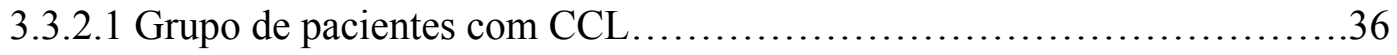

3.3.2.2 Grupo de Pacientes com DA........................................ 36

3.3.2.3 Grupo de Pacientes com DFT................................... 37

3.4 Procedimentos........................................................ 37

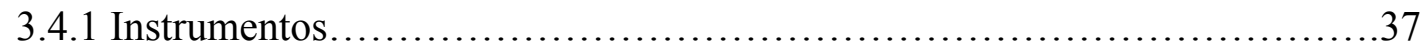

3.4.1.1 Testes de Rastreio..................................................... 37

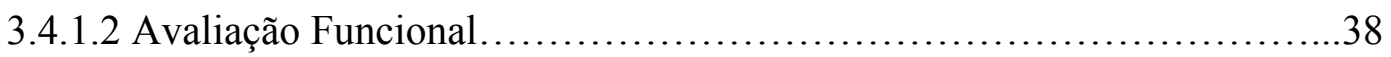

3.4.1.3 Avaliação do Humor.....................................................39

3.4.1.4 Avaliação Neuropsicológica...........................................39

3.4.1.5 Avaliação do Julgamento...........................................43

3.5 Adaptação...............................................................43

3.5.1 Processo de Tradução................................................43

3.5.2 Verificação da Acurácia.................................................44

3.6 Análises Estatísticas.................................................45

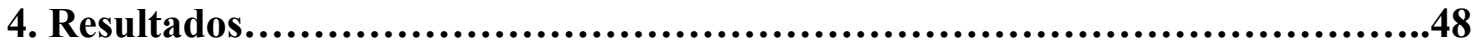

4.1. Análise Descritiva de Caracterização da Amostra..............................48

4.2 Análise Descritiva dos Resultados dos Testes.............................49

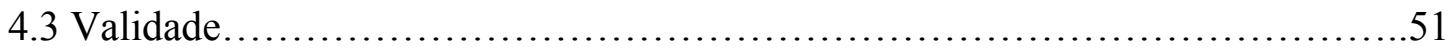

4.3.1 Validade Baseada na Estrutura Interna................................51 
4.3.1.1 Redução da Escala.................................................51

4.3.2 Validade Relativa ao Critério............................................53

4.3.3 Validade por Constructo Relacionado....................................54

4.4 Confiabilidade........................................................ 55

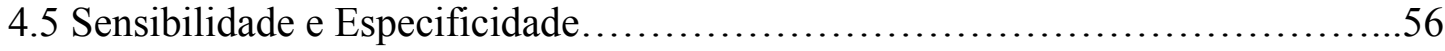

5. Discussãa.......................................................................60

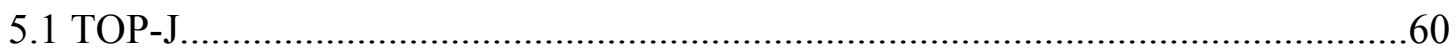

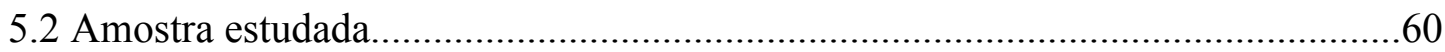

5.3 Evidências relacionadas à Validade Baseada na Estrutura Interna e Redução da

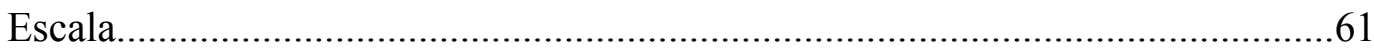

5.4 Evidências relacionadas à Validade de Critério......................................................63

5.5 Evidências relacionadas à Validade de Constructo................................................69

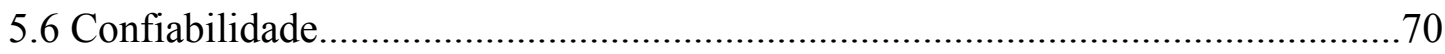

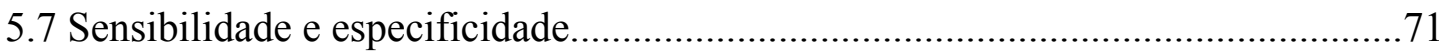

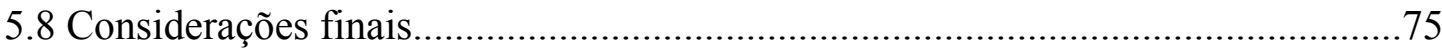

6. Conclusões......................................................................77

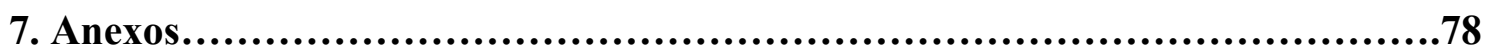

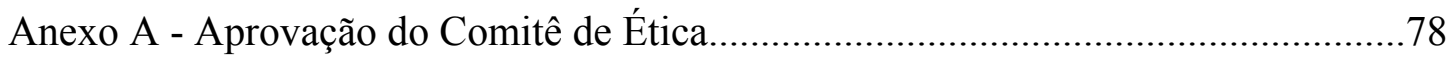

Anexo B - Termo de Consentimento Livre e Esclarecido..........................................79

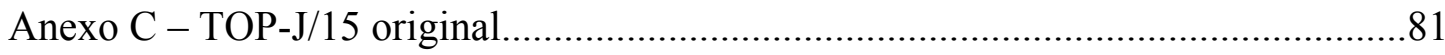

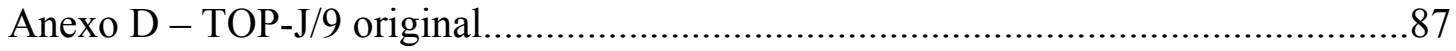

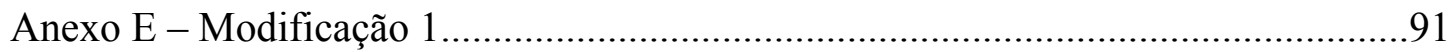

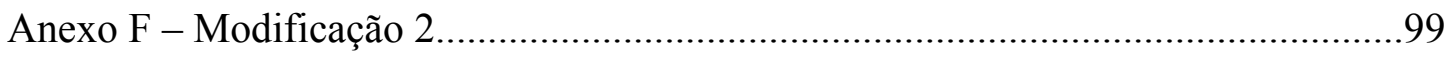

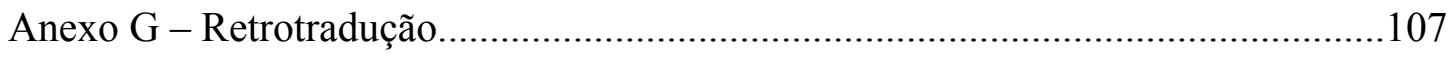

Anexo H - TOP-J/15 versão brasileira (TOP-J/15-Br) ….......................................115 
Anexo I - TOP-J/9 versão brasileira (TOP-J/9-Br) ...................................................122

8. Referências........................................................................126 


\section{LISTA DE SIGLAS E ABREVIATURAS}

ABVD - Atividades Básicas da Vida Diária

AIVD - Atividades Instrumentais da Vida Diária

APP - Afasia Progressiva Primária

BADS - Behavioral Assessment of the Dysexecutive Syndrome

BBRC - Bateria Breve de Rastreio Cognitivo

$\mathrm{CC}-$ Cognitive Complaints

CCL - Comprometimento Cognitivo Leve

CDR - Clinical Dementia Rating

CIND - Cognitive Impairment no Dementia

DA - Doença de Alzheimer

DFT - Demência Frontotemporal

DLB - Demência com corpos de Lewy

DLFT - Degeneração Lobar Frontotemporal

DP - Desvio Padrão

DR - Teste Desenho do Relógio

DV - Demência Vascular

EPCCE - Everyday Problems Test for Cognitively Challenged Elderly

EPT - Everyday Problems Test

FAS - Teste de Fluência Verbal Fonêmica das letras F, A e S

FV - Teste de Fluência verbal Semântica de categoria animais

GDS - Geriatric Depression Scale

HC - Healthy Control

ILS - Problem Solving Subscale of the Independent Living Scales 
IQCODE - Informant Questionnaire on Cognitive Decline in the Elderly

KPT - Kitchen Picture Test

MEEM - Mini Exame do Estado Mental

ML - Teste Memória Lógica

MoCA - Montreal Cognitive Assessment

MZ - Teste do Mapa do Jardim Zoológico

NAB JDC - Judgment Subtest of the Neuropsychological Assessment Battery

NACC - National Alzheimer's Coordinating Center

NCSE JQ - Judgment Questionnaire of the Neurobehavioral Cognitive Status Exam

QAF - Questionário de Atividades Funcionais de Pfeffer

RAVLT - Rey Auditory Verbal Learning Test

ROC - Receiving Operator Characteristic Curve

TMT - Trail Making Test

TOP-J - Test of Practical Judgment

TOP-J/15 - Test of Practical Judgment de 15 itens - original

TOP-J/9 - Test of Practical Judgment de 9 itens - original

TOP-J/15-Br - Test of Practical Judgment de 15 itens - versão brasileira

TOP-J/9-Br - Test of Practical Judgment de 9 itens - versão brasileira

WAIS - Weschsler Adult Intelligence Scale

WCST - Wisconsin Card Sorting Test 


\section{LISTA DE SÍMBOLOS}

$\%$ por cento

\pm mais ou menos

$\leq$ menor ou igual

$<$ menor

$>$ maior

$=$ igual 


\section{LISTA DE FIGURAS}

Figura 1 - Esquema Conceitual de Julgamento............................... 3

Figura 2 - Curvas ROC do TOP-J/15-Br................................ 58

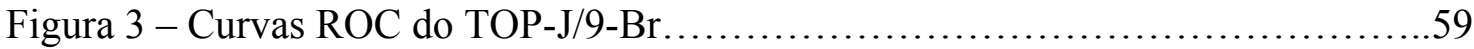




\section{LISTA DE TABELAS}

Tabela 1 - Estudos com testes de julgamento, exceto TOP-J, em pacientes com comprometimento cognitivo......................................17

Tabela 2 - Estudos com TOP-J em pacientes com comprometimento cognitivo......25

Tabela 3 - Perfil sociodemográfico dos participantes............................49

Tabela 4 - Comparação do desempenho entre os grupos nos testes aplicados........50

Tabela 5 - Comparação entre TOP-J/9 original e TOP-J/9-Br a partir do TOP-J/15..52

Tabela 6 - Análise Fatorial exploratória para um único fator......................53

Tabela 7 - Comparação do desempenho entre os grupos no TOP-J.................54

Tabela 8 - Correlação de Pearson entre TOP-J/15-Br e TOP-J/9-Br e demais testes..55

Tabela 9 - Análise da Consistência Interna do TOP-J/15-/Br e do TOP-J/9-Br.......56

Tabela 10 - Área sob a curva, pontos de corte, sensibilidade e especificidade.........57 


\section{RESUMO}

Capucho, PHFV. "Test of Practical Judgment" (TOP-J): Adaptação brasileira em amostra de individuos cognitivamente saudáveis, com comprometimento cognitivo leve e demência. [Dissertação]. São Paulo: Faculdade de Medicina, Universidade de São Paulo; 2015.

INTRODUÇÃO: Julgamento é a capacidade de tomar decisões após cuidadosa consideração das informações disponíveis, soluções possíveis, resultados prováveis e fatores contextuais. Do ponto de vista neuropsicológico, o conceito de julgamento envolve memória, linguagem, atenção, raciocínio e principalmente funções executivas. Perda de julgamento tem sido descrita em Comprometimento Cognitivo Leve (CCL) e demência. O Test of Practical Judgment (TOP-J) é uma medida originalmente americana, desenvolvida para avaliar o julgamento prático em adultos mais velhos. É um questionário aberto de 15 itens (TOP-J/15) ou de 9 itens (TOP-J/9) no qual os participantes escutam breves cenários sobre os problemas cotidianos e relatam em voz alta as soluções propostas. Este estudo teve como objetivos a adaptação do TOP-J para uso no Brasil, a elaboração de uma versão reduzida deste instrumento e verificação da acurácia de ambas a versões em amostra da população brasileira composta de controles cognitivamente saudáveis e pacientes com Comprometimento Cognitivo Leve (CCL), doença de Alzheimer (DA) e demência frontotemporal variante comportamental (DFT). MÉTODOS: A amostra foi composta por 85 indivíduos, com idade mínima de 50 anos e escolaridade mínima de 4 anos, sendo 24 controles, 26 CCL, 20 DA e 15 DFT. Os participantes foram submetidos a avaliação neuropsicológica e ao TOP-J. RESULTADOS: No TOP-J/15 versão brasileira (TOP-J/15-Br) e no TOP-J/9 versão brasileira (TOP-J/9-Br) (versão reduzida), o desempenho de controles foi estatisticamente superior ao desempenho de pacientes com CCL, DA e DFT e o desempenho de CCL foi superior ao de pacientes com DA e DFT. No TOP-J/15-Br, a confiabilidade verificada pelo alfa de Cronbach foi de 0,69 e o melhor ponto de corte para distinção de controles e pacientes foi de 30 (sensibilidade de 91,7\%; especificidade de $59 \%$ e área sob a curva de 0,80 ). No TOP-J/9-Br, o alfa de Cronbach foi de 0,68 e o melhor ponto de corte para distinção entre julgamento de controles e de pacientes foi 19, com sensibilidade de 79,2, especificidade de 72,1 e área sob a curva de 0,82 . CONCLUSÕES: O TOP-J/15-Br e o TOP-J/9-Br apresentaram características psicométricas robustas para o uso pretendido com amostra da população brasileira. Ambos foram capazes de identificar prejuízo de julgamento já em pacientes com CCL e diferenciaram julgamento de controles do julgamento de pacientes com boa sensibilidade e especificidade.

Descritores: Julgamento; Comprometimento cognitivo leve; Demência; Doença de Alzheimer; Demência frontotemporal; Testes neuropsicológicos. 


\begin{abstract}
Capucho, PHFV. Test of Practical Judgment (TOP-J): Brazilian adaptation in a sample of cognitively healthy individuals, mild cognitive impairment and dementia.
\end{abstract} [Dissertation]. São Paulo: "Faculdade de Medicina, Universidade de São Paulo"; 2015.

INTRODUCTION: Judgment is the ability to make sound decisions after careful consideration of available information, possible solutions, likely outcomes and contextual factors. From a neuropsychological perspective, the concept of judgment involves memory, language, attention, reasoning and specially the executive functions. Loss of judgment has been described in Mild Cognitive Impairment (MCI) and dementia. The Test of Practical Judgment (TOP-J) is an originally American measure created for evaluate practical judgment in older adults. It is a 15-item (TOP-J/15) or 9item (TOP-J/9) open-ended questionnaire in which participants listen to brief scenarios about everyday problems and report aloud their proposed solutions. This study aimed the adaptation of the TOP-J for use in Brazil, the development of a reduced version of this instrument and the verification of accuracy of both the versions in Brazilian sample composed of cognitively healthy controls and patients with mild cognitive impairment (MCI), Alzheimer's disease (AD) and frontotemporal dementia behavioral variant (FTD). METHODS: The sample consisted of 85 subjects with a minimum age of 50 years and minimum education of 4 years, being 24 controls, $26 \mathrm{MCI}, 20 \mathrm{AD}$ e 15 FTD. The participants were submitted to a neuropsychological assessment and TOP-J. RESULTS: In the TOP-J/15 Brazilian version (TOP-J/15-Br) and in the TOP-J/9 Brazilian version (TOP-J/9-Br) (reduced version), performance of controls was statistically better than the performance of $\mathrm{MCI}, \mathrm{AD}$ and FTD patients, and performance of MCI was statistically better than AD and FTD patients. In TOP-J/15-Br, the reliability verified by Cronbach's alpha was 0.69 and the best cutoff for distinction between controls and patients was 30 (sensibility of $91,7 \%$; specificity of $59 \%$ and area under the curve of 0,8$)$. In the TOP-J/9-Br, Cronbach's alpha was 0.68 and the best cutoff point for distinguishing between judgment of controls and judgment of patients is 19 , with a sensitivity of 79,2 , specificity of 72,1 and area under the curve of 0.82 . CONCLUSIONS: The TOP-J/15-Br and the TOP-J/9-Br showed robust psychometric characteristics for the intended use with a sample of the Brazilian population. Both were able to identify deficits of impaired of judgment already in patients with MCI and were able to distinguish judgment of controls from judgment of patients with good sensitivity and specificity.

Descriptors: Judgment; Mild cognitive impairment; Dementia; Alzheimer disease; Frontotemporal dementia; Neuropsychological tests. 


\section{INTRODUÇÃO}

\subsection{Julgamento}

Julgamento pode ser definido como a capacidade de tomar decisões após cuidadosa consideração sobre informações disponíveis, fatores contextuais, soluções possíveis e resultados prováveis (Rabin et al., 2008).

Conceitualmente, julgamento é muito relacionado com resolução de problemas e tomada de decisão, e frequentemente estes termos são utilizados de forma indistinguível na literatura. Entretanto, existem algumas diferenças conceituais entre eles.

Tomada de decisão refere-se ao processo inteiro de escolher uma ação (Hastie et al., 2001). Resolução de problemas, por outro lado, é uma operação que envolve fazer previsões probabilísticas sobre várias opções, a fim de identificar quais as opções mais viáveis (Collins et al., 1997). Julgamento refere-se aos componentes do processo de tomada de decisão relacionados a avaliar, estimar e inferir quais eventos irão aparecer, e quais serão as consequências para cada um dos possíveis resultados e o grau de satisfação com potenciais opções viáveis (Hastie, 2001). Desta forma, julgamento pode ser considerado como um dos últimos estágios da resolução ativa de um problema. Como exemplo, dizer que uma pessoa teve um julgamento ruim significa que ela tomou uma má decisão após consideração das informações, opções e contexto disponíveis (Rabin et al., 2008).

Davis (2012) discute que a decisão é uma ação (algo que fazemos ou escolhemos) e podemos decidir, sempre que houver mais de uma opção. Por outro lado, podemos julgar somente em situações em que, além da situação de escolha haja razões suficientes, em sentidos diferentes, para apostar em uma ação e não em outra. 
Para a execução de um bom julgamento é necessário atentar e compreender a situação, criar estratégias adequadas para abordar um problema, identificar objetivos, escolher uma ação em detrimento de outra, avaliar potenciais consequências entre determinados cursos de ação, inibir respostas inapropriadas, iniciar comportamentos propositais e monitorar os efeitos da solução escolhida (Hastie, 2001; Channon, 2004; Rabin et al., 2007; Margrett et al., 2010).

De uma perspectiva neuropsicológica, o conceito de julgamento envolve muitos aspectos cognitivos, incluindo memória (lembrar experiências passadas relevantes), linguagem (compreender aspectos verbais e não verbais, e comunicar a decisão a pessoas envolvidas), atenção, raciocínio (Allaire e Marsiske, 1999; Margrett et al., 2010) e especialmente de funções executivas (Woods et al., 2000; Duke e Kaszniak, 2000), que referem-se à capacidade de engajar em comportamentos complexos e objetivos, e que requer memória operacional, planejamento, organização, sequenciamento e abstração. Pessoas com déficits em funções executivas podem apresentar julgamento pobre por muitas razões, como tomar decisões impulsivas, focar somente em uma solução devido à flexibilidade mental reduzida ou por não considerar consequências a longo prazo (Channon, 2004).

Aspectos emocionais e sociais também tem influência no processo de julgamento prático, discutido acima, incluindo empatia, sensibilidade ao feedback social, percepção das consequências das ações escolhidas para outros e senso de responsabilidade e obrigações sociais, mas estão mais relacionados a julgamento moral ou social, definido como julgamento avaliativo da adequação do comportamento no contexto das percepções sociais de certo e errado (Moll et al., 2005).

Para fins didáticos, ao longo do texto o termo julgamento será utilizado ao se tratar de julgamento prático. 
A figura 1 ilustra e sintetiza a relação conceitual entre tomada de decisão, resolução de problemas, julgamento e decisão.

Figura 1. Esquema conceitual de Julgamento

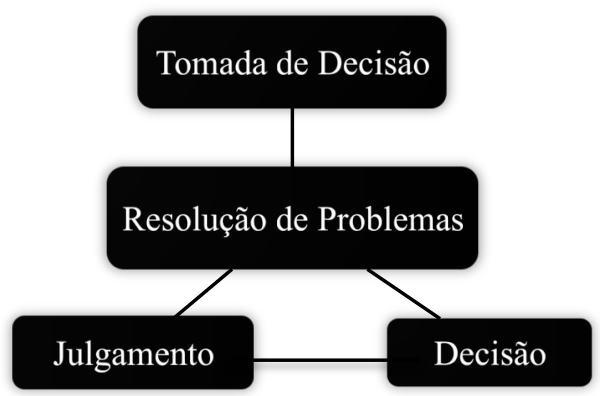

\subsection{Demência}

Demência é definida como uma síndrome caracterizada pelo declínio persistente de pelo menos duas funções cognitivas presentes nos seguintes domínios: memória, funções executivas, habilidades visuais-espaciais, linguagem e comportamento, e que interferem nas atividades sociais ou profissionais do indivíduo, independente de alterações do nível de consciência (Jack et al., 2011).

Demência é apontada como um dos maiores problemas de saúde, devido ao rápido crescimento na população idosa. No ano de 2005 foi estimado que 24.3 milhões de pessoas no mundo inteiro tinham demência, com 4,6 milhões de casos novos a cada ano (Ferri et al., 2005). Em uma revisão da prevalência de demência na América Latina foi encontrada uma prevalência de 7,1\% (Nitrini et al., 2009). Em revisão mais recente, realizada em 2012, a prevalência de demência na América Latina foi de 8,5\% e essa região foi identificada como a região com a maior prevalência de demência (World Health Organization, 2012). Lopes e Bottino (2002) verificaram, em uma revisão de 
estudos publicados de 1994 a 2000 em diversos países, que a prevalência de demência varia de $1,17 \%$ na população de 65 a 69 anos a 54,83\% na população acima de 95 anos. No Brasil foram feitos alguns estudos sobre a prevalência de demência. Em estudos realizados com sujeitos acima de 60 anos, Lopes et al., 2012 encontraram prevalência de 12.5\% em Ribeirão Preto, e Bottino et al., 2008 encontraram a prevalência de 12,9\% em São Paulo. Em estudos realizados com sujeitos com idade mínima de 65 anos, Herrera et al., 2002 encontraram prevalência de 7,1\% em Catanduva, e Scazufca et al., 2008 encontraram prevalência de demência de 5,1\% em São Paulo.

\subsubsection{Doença de Alzheimer (DA)}

A DA tem início insidioso e critérios para o diagnóstico clínico incluem: funcionamento cognitivo geral prejudicado determinado por exames clínicos e documentado pelo MEEM, Blessed Dementia Scale ou avaliações similares e confirmada por testes neuropsicológicos; déficits em duas ou mais áreas cognitivas; declínio progressivo de memória e outras funções cognitivas; ausência de distúrbios de consciência; início entre 40 e 90 anos (predominantemente depois dos 65 anos); e ausência de doenças sistêmicas ou outras doenças cerebrais que podem independentemente causar declínio progressivo na memória e cognição (McKhann et al., 1984). No Brasil, Herrera et al. (2002) verificaram que a prevalência de DA é de $55 \%$ dos casos de demência. Recentemente, foram realizadas modificações nos critérios diagnósticos da DA. As principais alterações incluem denominação demência da DA, visto que DA admite a fase pré-clínica e o Comprometimento Cognitivo Leve (CCL) devido à DA; não existe mais a obrigatoriedade do comprometimento da memória; a avaliação neuropsicológica é necessária quando a anamnese e a avaliação cognitiva realizada pelo médico forem insuficientes para o diagnóstico; o início não é mais limitado 
dos 40 aos 90 anos, e foi estipulada a necessidade de exame de imagem para excluir outras etiologias ou co-morbidades. A inclusão de biomarcadores, no diagnóstico, foi recomendada por enquanto somente em pesquisa clínica (Frota et al., 2011).

\subsubsection{Demência Frontotemporal}

A Degeneração Lobar Frontotemporal (DFLT) engloba a Demência frontotemporal (DFT) variante comportamental, a afasia progressiva primária (APP) e suas três variantes clínicas: agramática, semântica, e logopênica (Hodges e Miller, 2001; Rogalski et al., 2011; Harciarek e Kertesz, 2011).

Dentre estas, a DFT variante comportamental é a mais frequente, embora poucos estudos tenham investigado a sua prevalência. No Brasil, Herrera et al., 2002, encontraram taxa de prevalência de $0,2 \%$ em um estudo populacional realizado em Catanduva, com sujeitos com idade mínima de 65 anos. A baixa prevalência observada pode ser devida ao fato de que nesta amostra a idade mínima foi de 65 anos, e a DFT variante comportamental é mais frequente em indivíduos adultos mais jovens (Piguet et al., 2011, Josephs et al., 2011).

Critérios para o diagnóstico de DFT variante comportamental incluem deterioração progressiva do comportamento e/ou cognição observada na história; desinibição comportamental precoce; apatia ou inércia precoce; perda precoce de simpatia ou empatia; comportamentos perseverativos, estereotipados ou compulsivos/ritualísticos precoces; hiperolalidade e alterações na dieta; perfil neuropsicológico com déficits executivos e relativa preservação da memória e habilidades visuais-espaciais; declínio funcional significativo; resultados de exames de imagem com atrofia frontal ou temporal anterior, observados em neuroimagem estrutural (ressonância magnética ou tomografia computadorizada) e hipoperfusão ou 
hipometabolismo frontal e/ou temporal anterior em exames de neuroimagem funcional (tomografia por emissão de fóton único/SPECT ou tomografia por emissão de positrons/PET) (Rascovski et al., 2011). Entretanto, estes exames podem ser normais nas fases iniciais da doença (Eslinger et al., 2012).

A diferenciação de DA e DFT variante comportamental pode ser difícil. Ambas as desordens produzem uma síndrome demencial progressiva que pode incluir disfunção executiva e alteração comportamental, embora essas anormalidades sejam mais características da DFT variante comportamental precoce (Rascovsky et al., 2007), em que há relativa preservação da memória episódica, diferentemente do que ocorre na DA (Torralva et al., 2007; Gleichgerrcht et al., 2011). Em uma revisão realizada por LimaSilva et al., 2013, os autores observaram que DFT variante comportamental tem maior comprometimento funcional quando comparados com outros tipos de DLFT ou DA, e que o comprometimento cognitivo foi associado com medidas de funcionalidade nestes pacientes.

\subsection{Comprometimento Cognitivo Leve}

O Comprometimento Cognitivo Leve (CCL) é uma condição clínica inicialmente usada para classificar indivíduos em estágio intermediário entre normal e demência, particularmente DA. Critérios diagnósticos inicialmente propostos incluíam: queixas de memória, atividades diárias preservadas, função cognitiva geral intacta, comprometimento de memória além do esperado para a idade e ausência de demência (Petersen et al., 1999). Posteriormente, CCL foi definido como uma entidade heterogênea de acordo com a apresentação clínica, dividida em: CCL amnéstico único domínio, CCL não amnéstico único domínio, CCL amnéstico múltiplos domínios e CCL não amnéstico 
múltiplos domínios. Para cada subtipo pode haver múltiplas etiologias ou causas, além de DA (Petersen et al., 2004).

Os critérios iniciais foram revisados e modificados em consenso internacional. De acordo com Winblad et al., 2004, os critérios diagnósticos para CCL são: não normal e não demente (não preenche critérios para demência); evidência de deterioração cognitiva por auto-relatos ou relatos de informantes, comprometimento objetivo em tarefas cognitivas e/ou evidência de declínio ao longo do tempo em tarefas cognitivas objetivas e atividades da vida diária preservadas, e comprometimento mínimo em tarefas instrumentais complexas.

Em revisão sobre prevalência e incidência de CCL no Brasil, Brucki (2013) observou que alguns autores de estudos epidemiológicos têm adotado os critérios de Cognitive Impairment no Dementia (CIND) (Graham et al., 1997), em que o indivíduo tem déficit cognitivo sem perda de funcionalidade, sem necessidade de confirmação por um companheiro ou avaliação a longo prazo. A prevalência de comprometimento cognitivo incluindo CCL e CIND varia entre 7,7\% e $25,2 \%$.

Em uma meta análise recente, a prevalência de CCL variou de 3\% a 42\% e a prevalência de CIND variou de 5,1\% a 35,9\% (Ward et al., 2012). Maioli et al. (2007) após avaliação longitudinal de 52 pacientes com CCL observaram uma incidência de demência de $28,8 \%$, com DA respondendo por $53,3 \%$ dos casos, DA com doença cerebrovascular respondendo por $33,4 \%$ dos casos e DFT respondendo por $13,3 \%$ dos casos, com taxa de conversão de CCL para demência de 23,8\%.

Em um estudo populacional realizado em Porto Alegre com sujeitos de idade mínima de 68 anos, sendo 21 CCL e 220 cognitivamente intactos, Godinho et al. (2012) observaram que a prevalência de CCL foi de 6,1\%. Dentre estes, 38\% evoluíram para DA provável, 24\% permaneceram estáveis e 38\% melhoraram a cognição, retornando a 
performance prévia ao longo do follow-up, que variou de 19 a 70 meses. A taxa anual de conversão para DA foi de $8,5 \%$ e a taxa de reconversão foi $8,5 \%$.

César et al. (2014) realizaram um estudo epidemiológico na cidade de Tremembé, do qual participaram 20\% dos sujeitos com idade mínima de 60 anos, totalizando 738 foram, selecionados aleatoriamente; a prevalência de demência foi $17,5 \%$ e a prevalência de CIND foi $19,5 \%$.

\subsection{Avaliação de julgamento}

Rabin et al. (2008) realizaram uma pesquisa online para avaliar práticas e perspectivas de neuropsicólogos sobre avaliação de julgamento. Os participantes foram selecionados randomicamente dentre membros da Sociedade Internacional de Neuropsicologia e da Academia Nacional de Neuropsicologia, que possuíam titulação de doutores e moravam nos Estados Unidos ou Canadá. Eles foram solicitados a responder perguntas como: frequência com que julgamento é avaliado nas avaliações neuropsicológicas, em quais populações de pacientes, quais os instrumentos mais utilizados e o que pensam sobre a necessidade de medidas adicionais.

Com base nos resultados obtidos, a maioria dos entrevistados $(\mathrm{N}=290)$ relatou que "muitas vezes" ou "sempre", avaliam julgamento em pacientes com traumatismo craniano (89\%), demência (87\%) e distúrbios psiquiátricos (70\%). As técnicas mais frequentes de avaliação de julgamento são entrevistas clínicas com o paciente, testes formais de avaliação neuropsicológica, entrevistas com acompanhantes, escalas subjetivas de avaliação de comportamentos relevantes e observações diretas. Os testes mais utilizados para avaliar julgamento foram: Compreensão da Weschsler Adult Inteligence Scale (WAIS-III) (39\%), Wisconsin Card Sorting Test (WCST) (36\%), Semelhanças-WAIS-III (19\%), Judgment Questionnaire subtest of the Neurobehavioral Cognitive Status Exam 
(NCSE JQ) (Northern California Neurobehavioral Group, 1988) (14\%) e Judgment Subtest da Neuropsychological Assessment Battery NAB JDG (6\%). Os autores discutem que as três medidas mais utilizadas (Compreensão, WCST e Semelhanças) não foram desenvolvidas para avaliar julgamento per si, mas para avaliar a capacidade de lidar com resolução de problemas gerais e conhecimento de aspectos básicos de segurança e higiene. Aproximadamente $90 \%$ dos participantes responderam que é necessária a criação de medias adicionais específicas para a avaliação de julgamento.

Para avaliação de resolução de problemas, muitas medidas neuropsicológicas tradicionais têm sido usadas, principalmente com o objetivo de avaliar funções executivas (Farias et al., 2006; Espinosa et al., 2009). Entretanto, medidas não ecológicas podem subestimar o grau de dificuldades cotidianas, fornecendo pistas e informações relevantes, que muitas vezes não estão disponíveis na vida real (Channon e Crawford, 1999). Atividades Instrumentais da Vida Diária (AIVD) são intimamente relacionadas com resolução de problema cotidiano e alguns estudos usam escalas de AIVD (usualmente respondidas por informantes) para identificar habilidades de resolução de problemas (Farias et al., 2006; Kounti et al., 2006). No entanto, estas medidas podem não fornecer um meio adequado para avaliar a qualidade de comportamento durante o julgamento prático cotidiano, que exige que o sujeito busque informações e determine relevâncias antes de tomar uma decisão (Channon e Crawford, 1999).

Dentre as medidas que avaliam constructos que se sobrepõem a julgamento, podese citar o Predicaments Task (Channon e Crawford, 1999), Everyday Problem Solving Inventory (Cornelius e Caspi, 1987), Reflective Judgment Dilemmas (Kajanne, 2003), Practical Problems Test (Denney e Pearce, 1989), Everyday Cognition Battery (Allaire e Marsiske, 1999), Everyday Problems Test (EPT) (Artistico et al., 2003) e Everyday Problems Test for Cognitively Challenged Elderly (EPCCE) (Willis et al., 1998). Estes 
testes, contudo, foram desenvolvidos para fins de pesquisa, dispõem de pouca informação sobre propriedades psicométricas e não são rotineiramente utilizados por neuropsicólogos (Rabin et al., 2008). Além disso, somente o EPT e EPCCE foram estudados em adultos mais velhos com comprometimento cognitivo (Capucho e Brucki, 2011).

Algumas escalas têm sido desenvolvidas para avaliar a habilidade de consentir a tratamentos médicos ou pesquisas, como MacArthur Competency Assessment Tool for Treatment (Grisso et al., 1997), Capacity to Consent to Treatment Instrument (Marson et al., 1995), Hopkins Competency Assessment Test (Janofski et al., 1992), Aid to Capacity Evaluation (Etchells et al., 1999) e Assessment of the Capacity for Everyday Decision Making (Lai e Karlawish, 2007). Essas medidas provém informações úteis sobre fatores relacionados a decisões sobre cuidados médicos. Contudo, são específicas a situações particulares. Pacientes que apresentam comprometimento na capacidade de tomar decisões relacionadas ao tratamento médico, não necessariamente apresentam comprometimento na capacidade de fazer julgamentos e tomar decisões relacionadas a outros aspectos de suas vidas (Lim e Marin, 2011).

Atualmente, existem apenas 5 testes de julgamento com dados psicométricos disponíveis para uso em população adulta/idosa: 1) NCSE JQ, 2) Subescala Resolução de Problemas da Independent Living Scales (ILS) (Loeb, 1996), 3) NAB JDC (Stern e White, 2003), o Kitchen Picture Test (KPT) (Mansbach et al., 2013) e o Test of Practical Judgment (TOP-J) (Rabin et al., 2007). 


\subsubsection{NCSE JQ}

O Neurobehavioral Cognitive Status Examination (NCSE) é uma bateria de testes que avalia sete domínios cognitivos: orientação, atenção, linguagem, memória, cálculos e raciocínio. O domínio raciocínio é dividido em dois subtestes: semelhanças e julgamento (Judgment Questionnaire/JQ). O NCSE JQ é designado a avaliar julgamento através de perguntas sobre o que o sujeito faria diante de quatro situações problemáticas.

Drane e Osato (1997) compararam o desempenho do NCSE JQ entre indivíduos controles e indivíduos com diagnóstico de demência segundo critérios do DSM-III-R, residentes em uma casa de repouso. Os participantes tinham idade mínima de 60 anos e escolaridade média de 12 anos. O tamanho da amostra e o subtipo de demência não foi especificado. O NCSE JQ não foi capaz de distinguir entre indivíduos com demência daqueles sem demência.

Em outro estudo, Woods et al. (2000) avaliaram 40 controles e 95 pacientes com DA, dividido em dois grupos: mais comprometido $(\mathrm{MEEM}<20)$ e menos comprometido (MEEM $\geq 20$ ). Os resultados indicaram que foi observada diferença estatisticamente significativa entre controles e o grupo mais comprometido, mas não entre controles e o grupo menos comprometido. A consistência interna medida pelo alfa de Cronbach foi de 0,04 para controles e de 0,46 para DA.

Rabin et al. (2007) observaram que os escores do NCSE JQ não foram diferentes entre grupos de controles sem queixa e com queixa, e pacientes com CCL e DA, com alfa de Cronbach de $0,07$.

Além disso, o NCSE JQ parece apresentar vieses na correção. Fouty et al., (2013) realizaram um estudo com 110 indivíduos cognitivamente saudáveis (45 homens, 65 mulheres) com objetivo de verificar influência de gênero e idade em determinado no item 3 do NCSE JQ, que consiste na seguinte pergunta: O que você faria se você chegasse em 
casa e descobrisse que um cano quebrado estava inundando cozinha?". Os indivíduos foram divididos em dois grupos de idade: 64 anos ou menos e 65 anos ou mais. Não houve diferença na pontuação entre os dois grupos de idade. Entretanto, os autores observaram que mulheres frequentemente davam respostas do tipo "chamaria encanador, chamaria o marido" pontuadas como 1, segundo o manual, e homens geralmente davam respostas do tipo consertaria a válvula", pontuadas como 2, de acordo com o manual. Esta diferença entre padrões de resposta foi estatisticamente significativa entre homens e mulheres, e os autores sugeriram correção por gênero para este item.

O manual do NCSE JQ dispõe de poucos dados normativos e oferece poucas orientações sobre administração, pontuação e interpretação (Rabin et al., 2007).

\subsubsection{Subescala Resolução de Problemas da ILS}

O ILS é composto por 2 subescalas: Informação de desempenho e Resolução de Problemas. A Subescala Resolução de Problemas é composta de 33 itens (situados em todas as subescalas) que avaliam raciocínio abstrato e julgamento necessário para a vida diária, como exemplo: "O que você faria se as luzes e a televisão da sua casa apagassem simultaneamente?”. A aplicação dura de 20 a 25 minutos (Revheim e Medalia, 2004).

No único estudo encontrado sobre aplicabilidade da Subescala Resolução de Problemas da ILS em pacientes com demência, Baird et al. (2006) avaliaram 83 adultos divididos em cognição normal, comprometimento cognitivo sem demência, demência leve e demência moderada. Observou-se que este teste não foi capaz de diferenciar pacientes com comprometimento cognitivo sem demência daqueles com demência leve, mas diferenciou estes grupos daqueles com cognição normal (melhor desempenho) e demência moderada (pior desempenho). 


\subsubsection{NAB JDC}

A Neuropsychological Assessment Battery (NAB), (Stern \& White, 2003) é uma bateria completa de testes neuropsicológicos desenvolvida para abranger atenção, linguagem, memória, habilidades viso-espaciais e funções executivas. Foi padronizada nos Estados Unidos em amostra de 1.448 adultos com idades entre 17-97 anos (Gavett et al., 2012). O subteste Julgamento está incluso no módulo funções executivas e é composto por 10 itens. A consistência interna $(\alpha$ de Cronbach $=0,45)$ e estimativas de confiabilidade teste -reteste foram baixos $(r=0,37$; média de tempo entre teste e reteste superior a 6 meses) para os adultos com maior idade.

Desde 2003, somente três estudos (Zgaljardic et al., 2011; Gavett et al., 2012; MacDougall \& Mansbach, 2013) avaliaram a utilidade do NAB-JDC.

Zgaljardic et al. (2011) investigaram a validade ecológica do NAB - JDG em uma amostra de adultos jovens de 47 pacientes com traumatismo cerebral moderada a grave e observaram que os escores NAB - JDG correlacionaram-se significativamente com pontuação do Mayo- Portland Adaptability Inventory -4, uma medida de avaliação de consequências de traumatismo cerebral.

Em um estudo longitudinal, Gavett et al. (2012) verificaram dados de arquivo de 276 adultos (65 DA possível ou provável e 211 com outros diagnósticos não especificados) do Centro de Doença de Alzheimer da Universidade de Boston, com idade mínima de 50 anos e escolaridade mínima de 6 anos. Os autores verificaram que o grupo DA obteve desempenho significativamente pior em todas as medidas do NAB e encontraram um ponto de corte menor ou igual a 12 como o resultado de melhor equilíbrio entre sensibilidade $(0,61)$ e especificidade $(0,88)$ no NAB JDC. Somente este estudo avaliou utilidade diagnóstica do NAB-JDC para identificar idosos com DA. 
MacDougall e Mansbach (2013) analisaram as características psicométricas do NAB-JDG em 82 adultos, com idade mínima de 60 anos e escolaridade mínima de 5 anos, residentes em instituição de longa permanência, sendo 26 CCL e 56 com diagnóstico de demência, sem especificação do subtipo. Indivíduos com CDR=0,5 e 1 foram inclusos em um único grupo titulado demência leve $(\mathrm{N}=31)$. Os demais 25 pacientes foram inclusos no grupo demência moderada. Houve diferença estatisticamente significativa entre o desempenho de indivíduos capazes de consentir ao estudo e aqueles não capazes de consentir ao estudo. Os autores não relataram se houve diferença estatisticamente significativa entre CCL e demência. A consistência interna dos escores NAB - JDG nesta amostra, estimada pelo alfa de Cronbach, foi de 0,83. Apesar da consistência interna observada nesse estudo ter sido alta, o NAB - JDG aborda principalmente questões de segurança e de higiene básicas, em vez de julgamento de alto nível (Mansbach et al., 2013) .

\subsubsection{KPT}

O Kitchen Picture Test (KPT) (Mansbach et al., 2013) é um teste visual de julgamento, desenvolvido recentemente. O KPT descreve uma cena em que uma mulher está falando ao telefone, sem conhecimento de três acontecimentos perigosos que se passam em torno dela: (1) Um jovem garoto em direção ao fogão com uma panela em chamas, (2) uma faca afiada e prato quebrado no chão e (3) leite derramando de uma geladeira aberta. Os participantes são solicitados a identificar as três situações-problema (0-3 pontos possíveis), classificar a ordem de periculosidade (0-2 pontos) e proporcionar resoluções (0-3 pontos). A pontuação para a identificação das três situações-problema é baseada na identificação correta. A pontuação para classificação da periculosidade é baseada na ordem correta (o jovem garoto se aproximando da panela, faca afiada e leite 
derramado). A pontuação para intervenções de resolução de problemas é baseado no cumprimento das normas básicas de estratégias de resolução de problemas apropriada.

Para o desenvolvimento e validação do KPT, Mansbach et al. (2013) realizaram dois estudos independentes. O primeiro foi realizado com amostra de 99 sujeitos residentes em instituição de longa permanência, dos quais $49 \%$ tinham demência e 51\% não tinham demência (outras alterações cognitivas, não especificadas). A idade mínima foi 60 anos e $84 \%$ tinham pelo menos 12 anos de educação. O alfa de Cronbach foi 0,88 .

O segundo estudo consistiu em análise retrospectiva do desempenho de 163 indivíduos, dentre os quais 58 eram residentes de um asilo, enquanto que 64,4\% (n=105) estavam em reabilitação em serviços especializados de enfermagem. Os participantes tinham idade a partir de 60 anos e $80 \%$ tinham pelo menos 12 anos de educação. Dos 159 pacientes, $67 \%$ preencheram os critérios para a demência, enquanto 31 \% não tinham um diagnóstico de demência. $\mathrm{O}$ alfa de Cronbach obtido foi 0,93, a área sob a curva ROC para o KPT foi 0,89 , e foi obtida uma nota de corte de 7 , com sensibilidade de 0,85 e especificidade de 0,72 . Os pacientes com demência tiveram pontuação significativamente menor que os pacientes sem demência e os participantes com escolaridade $<12$ anos obtiveram pontuação significativamente mais baixa no KPT do que aqueles com escolaridade $\geq 12$ anos, o que aponta para a influência da escolaridade neste teste, apesar de ser visual. Em ambos os estudos, os autores não especificaram qual a desordem cognitiva do grupo de pacientes não dementes e nem o subtipo de demência dos pacientes, e não incluíram controles (Mansbach et al., 2013).

Segundo os autores, o KPT não é uma medida adequada para as pessoas com deficiências visuais, avalia julgamento de uma maneira geral, o critério de classificação das respostas não é objetivo, pode haver baixa confiabilidade inter-examinadores de diferentes áreas da saúde, e é provável que os dados psicométricos não sejam 
generalizáveis para outras populações, visto que os participantes de ambos os estudos foram encaminhados para a avaliação por profissionais de instituições de longa duração (Mansbach et al., 2013).

Em resumo, pode-se observar na tabela 1 os estudos desenvolvidos com os instrumentos descritos acima, em pacientes com comprometimento cognitivo. 
Tabela 1. Estudos com testes de julgamento, exceto TOP-J, em pacientes com comprometimento cognitivo.

\begin{tabular}{|c|c|c|c|}
\hline Estudo & Amostra & Teste & Resultados \\
\hline Drane e Osato, 1997 & $\begin{array}{llr}\text { Controles e demência definidos } \\
\text { pelo } \quad \text { DSM-III-R r sem } \\
\text { especificação da etiologia }\end{array}$ & NCSE JQ & $\begin{array}{l}\text { Não houve diferença } \\
\text { estatisticamente significativa do } \\
\text { desempenho entre os grupos. }\end{array}$ \\
\hline
\end{tabular}

Woods et al., 2000

Baird, 2006

Rabin et al., 2007

Gavett et al., 2012

MacDougall e Mansbach, 2013

Mansbach et al., 2013
40 controles e 95 DA divididos em mais comprometidos (MEEM <20) e menos comprometidos $($ MEEM $\geq 20)$

83 adultos mais velhos divididos em cognição normal, limítrofe, demência leve e demência moderada

$\mathrm{N}=134$ sujeitos, sendo 26 DA, 34 CCL, 39 sujeitos com cognição normal com queixa (CC) e 35 controles sem queixas (HC)

$\mathrm{N}=276$ (65 DA possível ou provável e 211 com outros diagnósticos não especificados)

$\mathrm{N}=82$ (26 CCL e 56 demência com etiologia não especificada). Indivíduos com $\mathrm{CDR}=0,5$ foram inclusos no grupo demência leve $(\mathrm{N}=31)$ e 25 foram inclusos no grupo demência moderada

Estudo 1: $\quad \mathrm{N}=99 \quad(49 \%$ com demência e 51\% com outras alterações, não especificadas) Estudo 2: $\mathrm{N}=163$ (159 pacientes - 67\% com demência e $31 \%$ sem demência)
NCSE JQ Observou-se diferença estatisticamente significativa entre controle e DA mais comprometido, mas não entre controles e DA de alto funcionamento. Alfa de Cronbach foi de 0,04 para controles e de 0,46 para DA.

ILS Desempenho similar entre cognição limítrofe e demência leve. Pacientes com demência moderada tiveram pior desempenho e sujeitos com cognição normal tiveram melhores escores em todas as subescalas, incluindo Problem Solving Subescale.

NCSE JQ Não houve diferença estatisticamente significativa entre os grupos. Alfa de Cronbach de 0,07 para o NCSE JQ.

NAB JDC Com nota de corte de 12, sensibilidade de 0,61 e especificidade de 0,88 , o NAB JDC mostrou-se útil na identificação de idosos com DA.

NAB JDC Houve diferença de desempenho entre o grupo capaz e o não capaz de consentir ao estudo. Alfa de Cronbach $=0,83$.

KPT

Estudo 1: Alfa de Cronbach $=0,88$. Pacientes com demência tiveram pior desempenho.

Estudo 2: Alfa de Cronbach $=$ 0,93, área sob a curva $=0,89$, ponto de corte $=7$, sensibilidade $=$ 0,85 e especificidade $=0,72$. Pacientes com demência e sujeitos com escolaridade $<12$ anos tiveram desempenho significativamente inferior. 


\subsubsection{Test of Practical Judgment (TOP-J)}

Em resposta à necessidade de uma medida clinicamente relevante para a avaliação de julgamento em adultos mais velhos, Rabin et al. (2007) desenvolveram o Test of Practical - Judgment (TOP-J), um questionário de 15 (TOP-J/15) ou 9 (TOP-J/9) perguntas abertas, nas quais os participantes escutam a breves cenários sobre problemas diários e relatam em voz alta as suas possíveis soluções (Rabin et al., $2013^{1}$ - trabalho não publicado, cedido pela autora). Estes cenários foram criados para serem facilmente entendidos e representativos dos tipos de problemas de julgamento encontrados pelos adultos mais velhos, embora complexos o suficientes para requererem habilidades cognitivas avançadas.

Os itens foram desenvolvidos através de revisão da literatura psicológica e neuropsicológica relacionada à avaliação clínica de julgamento resolução de problemas, tomada de decisão e processos executivos relacionados em adultos mais velhos. Neuropsicólogos foram questionados sobre o uso do termo julgamento prático e quais domínios esse termo poderia abarcar (Rabin et al., 2008). Adicionalmente, participantes e informantes do estudo Dartmouth de memória e envelhecimento (Dartmouth Memory and Aging Study), ocasionalmente mencionavam dificuldades com aspectos do julgamento, e estes comentários foram compilados, resultando em um processo de coleta de dados abrangente rendeu 4 domínios de conteúdo: segurança, social/ético, financeiro e médico (Rabin et al., 2007).

Depois de estabelecido o conteúdo dos domínios, os autores criaram um grupo de cenários com o objetivo que fossem ecológicos e representativos dos tipos de problemas

\footnotetext{
${ }^{1}$ Rabin L, Aronov A, Chi S, Fogel J, Charcape M, Borgos M, Saykin A. (Brooklyn College and The Graduate Center of City University New York - CUNY, Albert Einstein College of Medicine,). The Test of Practical Judgment (TOP-J): Updated Normative Data and Validation in a Demographically Diverse Group of Older Adults. (Presented at the Annual Meeting 410 of the International Neuropsychology Society -INS; 2013; Hawaii).
} 
de julgamento regularmente enfrentados pelos adultos mais velhos. Os itens deveriam ser simples e de fácil compreensão, mas complexos o suficiente para exigir funções cognitivas (por exemplo para resolução ativa de problemas e consideração sobre opções e potenciais consequências de diversas ações), minimizando a interferência do funcionamento emocional e conhecimento cristalizado. Para maximizar a validade de face e conteúdo, a maioria dos itens foi baseada em situações reais notificados pelos adultos mais velhos. Para evitar imposição/restrição de resposta, optou-se por um formato de resposta aberto no qual os participantes escutam breves cenários e relatam em voz alta as soluções propostas. Durante administração, o uso de instruções para orientar os participantes deve ser evitada. Os examinadores devem questionar respostas ambíguas, incompletas ou múltiplas respostas da seguinte forma: "Fale mais sobre isso " ou " Explique o que você quer dizer" (Rabin et al., 2007).

As respostas relatadas são escritas. Critérios de pontuação foram desenvolvidos com base em considerações teóricas e práticas. Foram desenvolvidas amostras de respostas que abrangessem uma ampla gama de possíveis relatos, com os mesmos elementos proeminentes. Os escores por resposta variam de 0 a 3 , com o maior valor indicando melhor julgamento. Respostas incomuns são pontuadas de acordo com seu grau de similaridade com o conteúdo específico ou significado geral da amostra de respostas. Instruções detalhadas são inclusas no protocolo de aplicação e o TOP-J/9 requer em média 10 minutos, para aplicação e pontuação (Rabin et al., 2007).

Os autores revisaram o conjunto inicial de 20 itens e retiraram 3 itens que eram redundantes ou confusos. A versão de 17 itens foi administrada por telefone em 45 participantes. Após este piloto de coleta de dados, os autores reavaliaram todos os itens do teste e critérios de pontuação e duas perguntas foram retiradas devido a problemas na administração, pontuação ou compreensão. Dos 15 itens restantes, seis itens foram 
posteriormente excluídos com base em achados de baixa carga na análise de fatores na unidade fator geral do TOP-J. Os 9 itens mantidos tinham carga fatorial superior a $0,4 \mathrm{e}$ foram ordenadas aleatoriamente no protocolo. O protocolo de teste foi distribuída a um grupo de 16 neuropsicólogos, que foram solicitados a indicar o domínio de julgamento melhor representasse cada item. Dado o número relativamente pequeno de itens e a sobreposição do conteúdo dos domínios para quase metade dos itens do teste, a prática recomendada é resumir todos os itens do TOP-J em um único score (Rabin et al., 2007).

Os autores avaliaram propriedades psicométricas do TOP-J/15 e do TOP-J/9.

A validação do TOP-J/15 foi realizada em amostra 119 indivíduos (Rabin et al., $2005^{2}$ - trabalho não publicado, cedido pela autora), subdivididos em 4 grupos: controles cognitivamente saudáveis sem queixas (health control - $\mathrm{HC})(\mathrm{N}=36)$, controles cognitivamente saudáveis com queixas cognitivas (cognitive complaints - CC) $(\mathrm{N}=33)$, pacientes com CCL $(\mathrm{N}=28)$ e pacientes com DA $(\mathrm{N}=22)$. Uma amostra de 45 sujeitos recebeu uma segunda administração do TOP-J 4 meses depois. A estabilidade testereteste foi 0,86 , confiabilidade entre avaliadores foi de 0,92 , e a consistência interna (alfa de Cronbach) foi de 0,68. O TOP-J/15 discriminou os grupos participantes: HC obtiveram pontuação significativamente maior (média $=37,6 \pm 3,9)$ do que CC (média $=$ $33,8 \pm 3,8$ ) e CCL (média $=34.1 \pm 4.2$ ), que por sua vez obtiveram pontuações significativamente maiores do que os indivíduos com DA leve (média $=28,4 \pm 6,1$ ). Não houve diferença estatisticamente significativa entre o grupo $\mathrm{CC}$ e o grupo CCL, o que sugere que que os CC possam representar uma condição pré-CCL. Os autores também compararam o desempenho no NSCE JQ entre os grupos e observaram que os escores

\footnotetext{
${ }^{2}$ Rabin LA, Borgos MJ, Saykin AJ, Root MD, Wishart HA, Flashman LA, Sprehn GC, Santulli RB. (Dartmouth Medical School). Judgment in Older Adults with AD, MCI, and Cognitive Complaints: Development and Preliminary Psychometric Evaluation of the Dartmouth-Rabin Judgment Questionnaire (DRJQ) (Presented at the 330th Annual Meeting of the International Neuropsychology Society; 2005; St. Louis, MO).
} 
não diferiram significativamente. A pontuação do TOP-J foi correlacionada com o desempenho em alguns testes de funcionamento executivo (número de erros perseverativos no WCST e no teste de fluência verbal fonêmica do D-KEFS). Os resultados indicaram que o TOP-J/15 é sensível aos estágios iniciais da demência, com pacientes com DA demonstrando acentuado comprometimento em relação ao grupo HC mais de 2 desvios-padrão (DP) abaixo da média. Apesar de CCL e CC terem obtido pontuação significativamente inferior ao grupo $\mathrm{HC}$, suas pontuações ainda estavam dentro dos limites clínicos (1 DP abaixo da média). Os dados apoiaram o uso do TOP-J nas avaliações dos idosos em vários estágios de comprometimento cognitivo.

Em um estudo realizado recentemente, Rabin et al., $2013^{1}$ reavaliaram a validade do TOP-J em um centro urbano amostra de idosos sem demência, com características demográficas diferentes da amostra normativa inicial (Rabin et al., 2005 ${ }^{2}$ ). Os participantes foram 210 idosos sem demência, com idade média de 82 anos, classificados em três grupos: 43 CCL, 62 CC e 105 HC. O coeficiente alfa de Cronbach do TOP-J/15 para esta amostra foi $0,60(\mathrm{p}<0,05)$. A confiabilidade teste-reteste para uma amostra de 19 participantes que receberam uma segunda administração do TOP-J um ano depois foi $0,78(\mathrm{p}<.001)$. Os grupos foram homogêneos para idade $(\mathrm{p}>0,05)$ e gênero $(\mathrm{p}>0,05)$, mas não para educação. Os grupos $\operatorname{HC}(14,8 \pm 3,1)$ e $\mathrm{CC}(15,2 \pm 3)$ foram mais escolarizados que o grupo $\operatorname{CCL}(12,7 \pm 3,4 ;$ p <.001). Análise de co-variância (ANCOVA), controladas para a educação, e comparações post-hoc indicaram que HCs obtiveram maior pontuação no TOP-J do que CCL. A análise fatorial exploratória identificou seis componentes, que representaram $55,9 \%$ da variância (todas as cargas fatoriais $>0,42$; apenas componentes com autovalores $>1$ foram mantidos). 
Baughman et al. (2011 - trabalho não publicado, cedido pela autora) avaliaram a estrutura fatorial do TOP-J/15 em uma população geriátrica de 118 veteranos de guerra (idade média $=75,1$ ). A análise de componentes principais produziu cinco componentes, que responderam por $56 \%$ da variância total. Os autores concluíram que o julgamento é um conceito multidimensional e que o TOP-J/15 oferece informações adicionais que podem ser negligenciadas no TOP-J/9, em uma população mais diversificada.

O TOP-J/9 também discriminou os grupos participantes (Rabin et al., 2007). O grupo $\mathrm{HC}$ (média= 23,0 $\pm 2,4$ ) teve desempenho estatisticamente melhor do que os grupos CC $(20,4 \pm 3,1)$ e CCL $(20,2 \pm 3,4)$, que por sua vez tiveram desempenho melhor que o grupo DA $(16,2 \pm 4,8)$. Não houve diferença estatisticamente significativa entre os grupos CC E CCL. A fidedignidade do TOP-J/9 foi verificada através da estabilidade teste-reteste $(0,86)$, confiabilidade inter-examinadores $(0,96)$ e consistência interna verificada com alfa de Cronbach de 0,63. Evidências de validade convergente foram demonstradas por correlações moderadas com constructos relacionados (ex. testes de funcionamento executivo, conhecimento cristalizado, linguagem e memória) ao passo que a validade discriminante foi demonstrada por baixas correlações com medidas envolvendo demandas executivas mínimas (ex. visuo-construção e funcionamento emocional).

Em outro estudo, Rabin e colaboradores (2009) examinaram a relação entre dados de neuroimagem estrutural e habilidade de julgamento em adultos mais velhos. Eles usaram morfometria baseada em voxel (VBM) para verificar áreas onde a redução da quantidade de substância cinzenta foi associada com escores no TOP-J/9. Participaram do estudo 120 indivíduos com idade igual ou superior a 60 anos, sendo 13 pacientes com

\footnotetext{
${ }^{3}$ Baughman, B.C., Young, J.C., Crouse, E.M., Yehyawi, N.T., Dowd, A., \& Hennessey, L.K. (2011, February). Exploring judgment in geriatric veterans: Factor structure of the Test of Practical Judgment $(T O P-J)$. (Presented at the 39th annual meeting for the International Neuropsychological Society; 2011; Boston, MA).
} 
DA, 34 pacientes com Comprometimento Cognitivo Leve (CCL), 34 controles saudáveis com queixas cognitivas e 39 controles saudáveis sem queixas cognitivas. Os resultados indicaram que baixo desempenho no TOP-J foi correlacionado com redução da densidade da substância cinzenta no giro frontal inferior esquerdo e em menor extensão com o giro frontal superior, proporcionando evidência para a sensibilidade do teste para a integridade de regiões pré-frontais do cérebro em indivíduos com risco para a demência.

Em um estudo com pacientes psiquiátricos, Borgos et al. (2006) (apud Rabin et al., 2007) observaram que indivíduos com diagnóstico de esquizofrenia, desordem esquizoafetiva, transtorno bipolar, transtorno de personalidade borderline e histórico de dependência química tiveram desempenho significativamente pior no TOP-J, comparados a controles.

Baldock et al. (2014) avaliaram o desempenho de 18 pacientes, sendo 9 DA e DFT, no TOP-J. Eles foram pareados com base nos escores do teste Montral Cognitive Assessment (MoCA). Foi encontrada diferença estatisticamente significativa de desempenho entre os dois grupos $(\mathrm{p}=, 027)$, com pior desempenho no grupo DFT.

Pickens et al. (2010) fizeram uma revisão de artigos publicados do ano de 2003 ao ano de 2009, com objetivo de identificar a medida mais efetiva para avaliar funções executivas em adultos com comprometimento cognitivo, verificado através das variáveis psicométricas das medidas. Os autores observaram que, dentre as 18 medidas inclusas, somente o TOP-J incluiu todos os testes estatísticos necessários para o desenvolvimento de uma escala, considerando análise de fatores, validade e fidedignidade.

Embora a validação inicial tenha sido nos Estados Unidos com amostra rural demograficamente homogênea (Rabin et al., 2005²), em estudo posterior com amostra urbana demograficamente diversa (Rabin et al., $2013^{1}$ ), o TOP-J-15 foi bem adequado e demonstrou consistência interna e confiabilidade teste-reteste adequadas ao longo de um 
período de 1 ano. Além disso, o TOP-J-15 distinguiu CCL de controles sem queixa, replicando os achados iniciais de um relativo comprometimento de julgamento em demência pré-clínica.

Em síntese, com exceção do TOP-J, as outras três medidas verbais de avaliação de julgamento fazem parte de baterias de testes maiores, o que pode limitar seu uso, visto que profissionais da saúde muitas vezes não têm o tempo ou a formação necessária para administrar baterias de testes maiores. Além disso, estudos têm revelado propriedades psicométricas limitadas para estas medidas e pouca acurácia para distinção entre julgamento de indivíduos sem comprometimento cognitivo daqueles com CCL e demência.

A tabela 2 mostra o resumo dos estudos realizados com o TOP-J em pacientes com demência e CCL. 
Tabela 2. Estudos com o TOP-J em pacientes com comprometimento cognitivo

\begin{tabular}{|c|c|c|c|}
\hline Estudo & Amostra & Teste & Resultados \\
\hline Rabin et al., $2005^{2}$ & 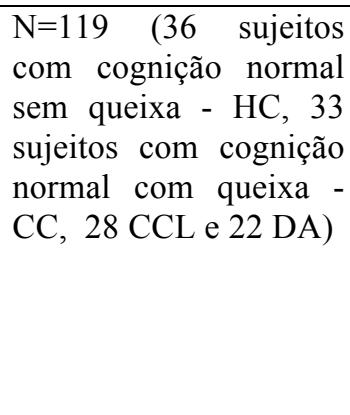 & TOP-J/15 & $\begin{array}{l}\text { Não houve diferença } \\
\text { estatisticamente significativa do } \\
\text { desempenho entre os grupos CC } \\
\text { e CCL. Estes grupos tiveram } \\
\text { pior desempenho que HC e } \\
\text { melhor desempenho que DA. } \\
\text { Estabilidade teste- reteste = } \\
0,86, \quad \text { confiabilidade entre } \\
\text { avaliadores }=0,92, \text { alfa de } \\
\text { Cronbach }=0,68 .\end{array}$ \\
\hline Rabin et al., 2007 & $\begin{array}{l}\mathrm{N}=134 \quad(26 \text { DA, } 34 \\
\mathrm{CCL}, 39 \mathrm{CC} \text { e } 35 \mathrm{HC})\end{array}$ & TOP-J/9 & $\begin{array}{l}\text { No TOP-J } / 9 \text { não houve diferença } \\
\text { estatisticamente significativa } \\
\text { entre CC e CCL. Estes grupos } \\
\text { tiveram escores piores que HC e } \\
\text { melhores que DA. Alfa de } \\
\text { Cronbach }=0,63 \text {, estabilidade } \\
\text { teste-reteste }=0,86 \text {, confiabilidade } \\
\text { inter-examinadores }=0,96 .\end{array}$ \\
\hline Rabin et al., 2009 & $\begin{array}{l}\mathrm{N}=120 \quad(13 \text { DA, } 34 \\
\mathrm{CCL}, 34 \mathrm{CC} \text { e } 39 \mathrm{HC})\end{array}$ & TOP-J/9 & $\begin{array}{l}\text { Foi avaliada a relação entre } \\
\text { neuroimagem estrutural e TOP- } \\
\text { J. Baixo desempenho no TOP-J } \\
\text { foi correlacionado com atrofia } \\
\text { do giro frontal inferior esquerdo } \\
\text { e em menor extensão com o giro } \\
\text { frontal superior. }\end{array}$ \\
\hline Rabin et al., $2013^{1}$ & $\begin{array}{l}\mathrm{N}=210 \quad(43 \mathrm{CCL}, 62 \\
\mathrm{CC} \text { e } 105 \mathrm{HC})\end{array}$ & TOP-J/15 & $\begin{array}{l}\text { Não houve diferença de } \\
\text { desempenho entre CCL e CC. } \\
\text { Estes grupos tiveram melhor } \\
\text { desempenho que controles. Alfa } \\
\text { de Cronbach }=0,6 \text {, confiabilidade } \\
\text { teste-reteste }=0,78 \text {. }\end{array}$ \\
\hline Baldock et al., 2014 & $\mathrm{~N}=18(9 \mathrm{DA}$ e $9 \mathrm{DFT})$ & TOP-J/9 & $\begin{array}{l}\text { Os pacientes foram pareados } \\
\text { com base nos escores do MoCA } \\
\text { Houve diferença estatisticamente } \\
\text { significativa no desempenho de } \\
\text { DA e DFT. }\end{array}$ \\
\hline
\end{tabular}

\subsection{Julgamento em demência}

Perda da capacidade de julgamento é comum em demência, quando as funções cognitivas que permitem o uso de comportamentos objetivos e habilidades de resolução de problemas mais complexos, como aqueles encontrados no trabalho, ambientes sociais, domésticos, em relações interpessoais são frequentemente afetadas e falham progressivamente (Duke e Kaszniak 2000, Channon, 2004; Karlawish et al., 2005). 
Embora alguns pacientes com demência possam executar atividades e tarefas rotineiras de forma adequada, a capacidade de resolver problemas mais complexos, como os encontrados no trabalho, ambientes sociais e nacionais e nas relações interpessoais geralmente são afetadas (Channon, 2004).

A funcionalidade, relacionada a AIVD e Atividades Básicas da Vida Diária (ABVD) tem a capacidade de discriminar entre indivíduos com demência de indivíduos sem demência. Déficits em AIVD surgem anteriormente aos déficits em ABVD em indivíduos com demência (Baird, 2006). O desempenho de resolução de problemas em pacientes com demência, mensurado por medidas neuropsicológicas tradicionais e escalas de AIVD (Kounti et al., 2006), bem como a capacidade de tomada de decisão, mensurada por testes de aposta (Delazer et al., 2007; Sinz et al., 2008) é comprovadamente afetado.

Willis et al. (1998) avaliaram o desempenho de 65 pacientes com DA leve e moderada, no Everyday Problems Test for Cognitively Challenged Elderly (EPCCE), um instrumento de 32 itens de resolução de problemas relacionados a finanças, medicações, transporte, uso de medicações, tarefas domésticas e preparo de comida. Houve diferença estatisticamente significativa entre os grupos, com os pacientes com menor prejuízo cognitivo demonstrando melhor desempenho.

Mayo et al. (2013) estudaram a relação entre declínio de julgamento, na resolução de problemas e nas atividades funcionais em indivíduos com demência com o objetivo de determinar se existe uma relação empírica entre o julgamento e resolução de problemas, estado funcional, cognição e subtipo de demência. Os dados de 3855 indivíduos com demência foram acessados a partir do National Alzheimer's Coordinating Center (NACC) Uniform Data Set (UDS). Para avaliar julgamento/resolução de problemas, foi utilizada a subescala Julgamento/Resolução de Problemas do Clinical Dementia Rating (CDR), que contem seis perguntas do tipo: Ele pode ele/ela pode lidar com uma 
emergência de família a) bem como antes, b) pior do que antes por causa de pensamento, ou c) pior do que antes (quando for por outro motivo). Os subtipos de demência incluídos foram: DA provável, DA possível, demência com corpúsculos de Lewy (DCL), demência vascular (DV), DFT e "outra“" categoria. Os resultados demonstraram cognição moderando uma forte relação entre o estado funcional e resolução problemas/julgamento entre os indivíduos com demência, com desempenho funcional menor prevendo pobre julgamento/resolução de problemas. Os escores Julgamento/resolução de problemas foram significativamente relacionados com escore do estado funcional e da cognição. Houve diferenças significativas entre os subtipos de demência em julgamento/resolução de problemas, estado funcional e cognição entre subtipos de demência. Os indivíduos com demência com corpos de Lewy (DLB) demonstraram mais prejuízo em julgamento/resolução de problemas, indivíduos com DV demonstraram maior comprometimento funcional e as piores pontuações nos testes cognitivos foram observados em DA provável e DV. A associação entre o julgamento/resolução de problemas e estado funcional foi mais forte para indivíduos com maus resultados em cognição. Não foram observadas diferenças estatisticamente significativas entre DA e DFT em julgamento. Os autores apontam a complexidade da habilidade julgamento/resolução de problemas, que requerem coordenação de numerosas áreas do cérebro e ressaltam a importância de estudos futuros utilizando medidas mais detalhadas de julgamento/resolução de problemas, com propriedades psicométricas em amostra de adultos mais velhos ainda não diagnosticados com demência.

Dez estudos foram encontrados sobre o desempenho de pacientes com demência nos testes de julgamento. Em um estudo, o NCSE JQ não foi capaz de diferenciar entre controles e pacientes com demência leve (Woods et al., 2000) e em outros dois estudos 
(Drane e Osato, 1997; Rabin et al., 2007) este teste não foi capaz de diferenciar controles de pacientes com demência avançada.

O Problem Solving Subescale da ILS não diferenciou pacientes com comprometimento cognitivo sem demência de demência leve, mas diferenciou estes grupos daqueles com cognição normal e demência moderada (Baird, 2006).

No TOP-J, o desempenho foi similar entre pacientes com CCL e aqueles com queixas e cognição normal (CC), mas houve diferença estatisticamente significativa entre o desempenho de CCL daqueles com cognição normal sem queixas (HC) e de pacientes com DA (Rabin et al., 2005²; Rabin et al., 2007 e Rabin et al., 2013 ${ }^{1}$ ), bem como entre pacientes com DA e DFT (Baldock et al., 2014).

Gavett et al. (2012) e MacDougall e Mansbach (2013) avaliaram o desempenho de indivíduos com demência no NAB JDC. Os primeiros autores observaram que o grupo DA desempenhou significativamente pior que os demais pacientes sem diagnóstico especificado no NAB JDC; enquanto que MacDougall e Mansbach observaram que houve diferença estatisticamente significativa entre o desempenho de indivíduos capazes e incapazes de consentir ao estudo, mas não entre CCL e demência.

Mansbach et al. (2013) avaliaram o desempenho de indivíduos com demência no KPT. Os pacientes com demência tiveram pontuação significativamente pior que os pacientes sem demência.

Somente os estudos de Rabin et al. $\left(2005^{2}, 2007\right.$ e $2013^{1)}$ e de Baldock et al. (2014), com o TOP-J; e o estudo de Gavett et al. (2012) com o NAB JDC, investigaram o desempenho de pacientes com DA. 


\subsection{Julgamento em Comprometimento Cognitivo Leve}

Há evidências sugerindo que dificuldades em resolução de problemas mensurada com escalas AIVD ou medidas neuropsicológicas tradicionais (Farias et al., 2006, Kounti et al., 2006, Perneczky et al., 2006, Wadley et al., 2007) e tomada de decisão mensurada por testes de apostas (Zamarian et al., 2011) podem ser observadas em adultos com CCL.

A detecção de declínio na capacidade de julgamento, assim como no funcionamento diário desses pacientes dependem da sensibilidade do método de avaliação usado na detecção das alterações sofridas por esses pacientes (Farias et al., 2006).

Com relação às medidas de constructos que se sobrepõem a julgamento, dois estudos foram encontrados e demonstraram que as pessoas com CCL têm pior desempenho nessas habilidades, se comparado ao grupo controle. Burton et al. $\left(2009^{\mathrm{a}}\right)$ avaliaram 250 pacientes (158 controles e 92 CCL) no Everyday Problems Test (EPT), teste composto por estímulos impressos relacionados ao uso de medicamentos, preparação de refeições, uso do telefone, compras, gestão financeira, administração do lar e transporte. Controles obtiveram resultados significativamente melhores do que os pacientes com CCL. Em outro estudo, Burton et al. (2009b) avaliaram o desempenho no EPT em 304 indivíduos classificados como cognitivamente intactos e com comprometimento cognitivo sem demência. O grupo comprometido apresentaram escores significativamente menores que os controles.

Embora prejuízos em constructos relacionados a julgamento estejam presentes em CCL, somente quatro estudos (Rabin et al., 2005²; Rabin et al., 2007; Rabin et al., $2013^{1}$ e MacDougall \& Mansbach, 2013) investigaram especificamente a habilidade de julgamento nesta população, com os testes TOP-J/15 (primeiro e terceiro estudos), TOPJ/9 (segundo estudo), NCSE JQ (segundo estudo) e NAB JDC (quarto estudo). Somente o 
TOP-J (15 e 9 itens) foi capaz de diferenciar CCL de controles e indivíduos com demência.

\subsection{Justificativa}

Apesar de medidas de julgamento terem sido negligenciadas, nos últimos anos tem aumentado o número de estudos de investigação da habilidade de julgamento em pacientes com comprometimento cognitivo. Em uma revisão sobre instrumentos disponíveis para avaliar especificamente a capacidade de julgamento em adultos mais velhos, com diferentes graus de comprometimento cognitivo, Capucho e Brucki (2011) encontraram quatro instrumentos disponíveis de julgamento. Recentemente, uma nova medida de avaliação de julgamento (visual) (KPT) (Mansbach et al., 2013) foi desenvolvida. Além disso, quatro novos estudos foram realizados em pacientes com demência (Gavett et al., 2012 - NAB JDC, McDougall e Mansbach, 2013 - NAB JDC, Mansbach et al., 2013 - KPT, e Rabin et al., $2013^{1}$ - TOP-J/15) e outros dois estudos foram realizados com CCL (McDougall e Mansbach, 2013 - NAB JDC e Rabin et al., $2013^{1}-$ TOP-J/15).

O TOP-J fornece informações valiosas sobre o julgamento (incluindo aspectos sociais, médicos, financeiros, sociais e de segurança) cotidiano em situações reais utilizadas para fins de diagnóstico, para direcionar questões relacionadas a competência funcional e cognição, e para tratamento. Desde a sua introdução em 2005, o TOP-J tem sido amplamente usado em clínica e pesquisa nos EUA e no exterior, incluindo clínicas de distúrbio de memória, asilos, unidades de reabilitação, centros médicos, faculdades, serviços de neuropsicologia, consultórios particulares e ambientes forenses (Rabin et al., $\left.2013^{1}\right)$. 
Considerando a relação entre capacidade funcional e julgamento, bem como dificuldades de julgamento observadas já em pacientes com CCL, pesquisas adicionais sobre esta habilidade em adultos mais velhos com comprometimento cognitivo de variados graus devem ser realizadas, com medidas ecológicas que abranjam problemas similares aos vivenciados na realidade. Tais pesquisas poderão ser úteis para inferências sobre quais indivíduos são capazes de viver de forma independente e segura.

Julgamento prático em situações cotidianas é um aspecto importante que deve ser investigado nas avaliações neuropsicológicas de adultos e idosos. Este estudo se justifica pela necessidade de uma medida de avaliação de julgamento para a população brasileira adulta e idosa, cognitivamente saudável e com comprometimento cognitivo em graus variados. Embora a impressão clínica seja atualmente o padrão aceito para determinação destes aspectos, a ausência de um padrão ouro pode tornar o parecer clínico pouco confiável.

O uso do TOP-J em nossa população poderá auxiliar na predição de consequências negativas e planejamento de tratamento de pacientes com comprometimento cognitivo, na orientação de cuidadores e no suporte a juízes e outros profissionais em aspectos jurídicos de perícia, interdição e intervenções legais. 


\section{OBJETIVOS}

\subsection{Objetivo Geral}

- Adaptação do Test of Practical Judgment (TOP-J) para uso no Brasil.

\subsection{Objetivos Específicos}

- Analisar a validade baseada na estrutura interna do TOP-J/15 versão brasileira (TOP-J/15-Br);

- Analisar a validade de critério concorrente do TOP-J/15-Br;

- Analisar a validade por constructo relacionado do TOP-J/15-Br;

- Analisar a confiabilidade (consistência interna) do TOP-J/15-Br;

- Verificar a sensibilidade e especificidade do TOP-J/15-Br;

- Obter de uma escala reduzida, a partir do TOP-J/15-Br, por meio da análise fatorial e verificar sua validade (de estrutura interna, critério e constructo), confiabilidade, sensibilidade e especificidade;

-Sugerir pontos de corte que possibilitem distinguir os indivíduos controles de pacientes com CCL, DA e DFT, para ambas as versões. 


\section{MÉTODOS}

\subsection{Desenho do estudo}

Este trabalho é um estudo observacional, transversal, descritivo e analítico.

\subsection{Aspectos éticos}

O projeto de pesquisa referente a esse estudo foi aprovado pela Comissão de Ética para Análise de Projeto de Pesquisa (CAPPesq) da diretoria clínica do Hospital das Clínicas e da Faculdade de Medicina da Universidade de São Paulo (HCFMUSP), sob protocolo de pesquisa número 0076/11 (Anexo A).

Todos os participantes receberam informações detalhadas sobre o estudo e assinaram o Termo de Consentimento Livre e Esclarecido (TCLE) (Anexo B)

Foi feito o pedido de autorização para o uso do TOP-J. Inicialmente, a pesquisadora entrou em contato, via correio eletrônico com a autora do instrumento. Além da autorização, a autora enviou a versão original de 15 itens (TOP-J/15) (Anexo C), a versão reduzida original (TOP-J/9) (Anexo D), concordou com os objetivos do presente estudo e afirmou estar satisfeita com a população estudada, que possibilitará o uso do TOP-J em populações de culturas diferentes.

\subsection{Casuística}

A amostra deste estudo foi composta de 85 indivíduos, divididos em quatro grupos: controles, pacientes com CCL, DA e DFT.

Para todos os grupos foram definidos os seguintes critérios iniciais de inclusão e exclusão: 


\section{Critérios de Inclusão}

- Idade igual ou superior a 50 anos;

- Escolaridade igual ou superior a 4 anos.

\section{Critérios de Exclusão}

- Distúrbios visuais e/ou auditivos sem correção;

- Distúrbios de linguagem;

- Distúrbios osteomusculares que prejudiquem a realização dos testes;

- Alcoolismo ou dependência química;

- Transtornos psiquiátricos (depressão não tratada);

- Doenças crônicas não compensadas;

- Escore maior ou igual a 6 na Escala de Depressão geriátrica reduzida (GDS-15).

Não foram excluídos indivíduos com depressão tratada com antidepressivos em dose estável por três meses. Nos indivíduos com DA e DFT os pacientes foram incluídos usando suas medicações para controle de alterações cognitivas e comportamentais.

\subsubsection{Grupo Controle}

O grupo controle foi composto por 24 indivíduos, acompanhantes de pacientes ou voluntários pertencentes à comunidade.

\section{Critérios de Inclusão}

- Ausência de queixas de memória;

- Independência nas atividades de vida diária. 


\section{Critérios de Exclusão:}

- Escore no Mini Exame do Estado Mental (MEEM) menor que mediana por escolaridade proposta por Brucki et al. (2003): 1 a 4 anos de escolaridade - 25; 5 a 8 anos - 26; 9 a 11 anos - 28 e acima de 11 anos - 29;

- Escore menor ou igual a 5 evocação tardia da Bateria Breve de Rastreio Cognitivo (Nitrini et al., 1994; 2004; 2007);

- Escore no Questionário do Informante do Declínio Cognitivo no Idoso Informant Questionnaire on Cognitive Decline in the Elderly (IQCODE) (Jorm, 1994) maior que 3,41(Bustamante et al., 2003);

- Escore no Questionário de Atividades Funcionais (QAF) maior que 2 pontos (Pfeffer et al., 1982);

- Doença neurológica, uso de substância psicoativa, como benzodiazepínicos ou antipsicóticos.

\subsubsection{Grupo de pacientes}

Foram avaliados 61 pacientes, sendo 26 pacientes com diagnóstico de CCL (amnéstico ou não amnéstico- domínio único ou múltiplos domínios) e 35 pacientes com demência de intensidade leve, subdivididos em dois grupos: 20 com diagnóstico de DA provável e 15 com diagnóstico de DFT (variante comportamental).

Os pacientes foram atendidos e diagnosticados pelos membros do Grupo de Neurologia Cognitiva e do Comportamento (GNCC) do Departamento de Neurologia do Hospital das Clínicas da Faculdade de Medicina da Universidade de São Paulo, e do Centro de Referência em Distúrbios Cognitivos (CEREDIC) da Faculdade de Medicina da Universidade de São Paulo. 


\subsubsection{Grupo de pacientes com CCL}

\section{Critérios de Inclusão:}

- Presença de um informante capaz de fornecer dados consistentes;

- Diagnóstico de CCL baseado nos critérios de Winblad et al. (2004).

\section{Critérios de Exclusão:}

- Escore maior que 4 pontos no QAF.

\subsubsection{Grupo de pacientes com DA}

\section{Critérios de Inclusão}

- Presença de um informante capaz de fornecer dados consistentes;

- Diagnóstico de DA provável, de acordo com os critérios do National Institute of Neurological and Communicative Disorders and Stroke e Alzheimer's Disease and Related Disorders Association (NINCDS-ADRDA) (McKhann et al., 1984);

- Demência de intensidade leve de acordo com os critérios do Manual de Diagnóstico e Estatística de Transtornos Mentais, $3^{\mathrm{a}}$ Edição, revisada, (DSM-IIIR) (American Psychiatric Association, 1987).

\section{Critérios de Exclusão}

- Demência de intensidade moderada ou grave e/ou demência de outra etiologia. 


\subsubsection{Grupo de pacientes com DFT}

\section{Critérios de Inclusão}

- Presença de um informante capaz de fornecer dados consistentes;

- Diagnóstico de DFT variante comportamental segundo os critérios de Rascovsky et al. (2011);

- Demência de intensidade leve de acordo com os critérios DSMIII-R (American Psychiatric Association, 1987).

\section{Critérios de Exclusão}

- Demência de intensidade moderada ou grave e/ou demência de outra etiologia.

\subsection{Procedimentos}

\subsubsection{Instrumentos}

Todos os indivíduos foram avaliados com os seguintes instrumentos:

\subsubsection{Testes de rastreio}

- Mini-Exame do Estado Mental - MEEM (Folstein et al., 1975; Brucki et al., 2003)

Instrumento de rastreio do comprometimento cognitivo, de fácil e rápida administração. Possui itens que envolvem orientação temporal e espacial, memória imediata e de evocação tardia, cálculo, linguagem e capacidade visual-construtiva. 
- Bateria Breve de Rastreio Cognitivo (BBRC) (Nitrini et al., 1994; 2004; 2007)

Avalia percepção visual, nomeação, memória incidental, memória imediata, aprendizado, memória tardia e reconhecimento. São utilizados como interferência os seguintes testes: Fluência Verbal (categoria animais) (Brucki et al., 1997, Spreen et al., 2006) no qual é solicitado ao sujeito que fale o maior número possível animais em um minuto e o Desenho do Relógio (Sunderland, 1989), no qual solicita-se que o participante desenhe um relógio com todos os números e ponteiros marcando 2:45.

\subsubsection{Avaliação Funcional}

- Questionário de Atividade Funcional (QAF) (Pfeffer et al., 1982)

Este instrumento é composto por 10 questões, com 5 alternativas cada. $\mathrm{O}$ informante assinalar em cada questão, a alternativa que mais se assemelha ao desempenho do indivíduo na realização de atividades instrumentais de vida diária.

- Questionário ao informante sobre o declínio cognitivo do idoso (Informant Questionnaire on Cognitive Decline in the Eldery - IQCODE) (Jorm, 1994; Bustamante et al., 2003).

Escala com 16 questões relativas a funcionalidade, nas quais o informante é solicitado a lembrar de como o indivíduo era há 10 anos e comparar com o estado atual, assinalando uma dentre cinco opções: (1) Muito Melhor, (2) Melhor, (3) Não muito alterado, (4) Pior e Muito Pior (5). 


\subsubsection{Avaliação do Humor}

-Escala de Depressão Geriátrica reduzida (Geriatric Depression Scale/GDS15) (Yesavage et al., 1983; Almeida e Almeida, 1999). É solicitado ao indivíduo que responda "sim" ou "não" para 15 perguntas relacionadas ao humor, de acordo com o modo que tem se sentido nas últimas semanas.

\subsubsection{Avaliação Neuropsicológica}

\section{- Teste do Mapa do Jardim Zoológico (MZ) subteste da Behavioural} Assessment of the Dysexecutive Syndrome - BADS (Wilson et al., 1996; Canali et al., 2007; Armentano et al., 2012)

Este subteste é dividido em duas tarefas. Na primeira, o indivíduo recebe uma folha com ilustração de um jardim zoológico, com solicitações de lugares a serem visitados e regras a serem seguidas. Na segunda tarefa, a ilustração do jardim zoológico é a mesma, mas a ordem em que os lugares devem ser visitados é descrita. Em ambas as tarefas, o sujeito deve fazer traçados no papel, indicando os lugares visitados. A primeira tarefa visa avaliar a habilidade de planejamento do sujeito em situações minimamente estruturadas e a segunda tarefa avalia a habilidade em seguir uma estratégia imposta externamente. Este subteste funções executivas relacionadas ao planejamento e sequenciamento.

- Trail Making Test (TMT) (Spreen et al., 2006)

O teste é composto de duas partes, A e B. Na parte A o indivíduo deve conectar consecutivamente círculos numerados. Em outra folha (parte B), pede-se que o sujeito 
que conecte sequencialmente círculos com números e círculos com letras, de forma alternada. Em ambas as partes o tempo é cronometrado e o escore de cada etapa é o total de tempo, em segundos, utilizado pelo sujeito para completar a prova. A parte A avalia atenção sustentada e a parte B avalia a atenção dividida e funções executivas relacionadas à flexibilidade mental.

- Teste de Fluência Verbal para categorias fonêmicas (FAS) (Spreen et al., 2006)

Neste teste, é solicitado ao indivíduo que fale o maior número de palavras, no período de um minuto, que comecem a letra F, excluindo-se nomes próprios, a mesma palavra com sufixo diferente e números. Em seguida, o mesmo procedimento é utilizado com as letra A e S. A pontuação da prova é a soma do número de palavras admissíveis geradas para cada uma das letras. Este teste avalia linguagem (fluência) e funções executivas relacionadas à categorização e controle inibitório.

- Dígitos: subteste da Escala de Inteligência Wechsler para Adultos (WAIS-III) (Wechsler, 1981; Wechsler, 1997; Nascimento, 2004)

Neste subteste o examinador lê, em voz alta, sequências de números, em ordem de quantidade crescente, e o examinando é solicitado a repeti-las em ordem direta, na primeira parte, e em ordem inversa, na segunda parte do teste. A parte A ordem direta avalia atenção e memória imediata; e a parte B avalia funções executivas relacionadas à memória operacional (manipulação de informações). 
-Wisconsin Card Sorting Test (WCST) - versão reduzida (Nelson, 1976; Ávila, 2007)

$\mathrm{O}$ teste consiste em um baralho com desenhos de figuras geométricas coloridas. $\mathrm{O}$ examinando é solicitado a combinar 48 cartas-resposta, com 4 cartas-modelo. As cartas podem ser combinadas por cor, forma ou número. A modo com que as cartas devem ser combinadas não é dito e o examinador fala somente se a combinação está certa ou errada. O examinando deve realizar as combinações de acordo com o feedback dado pelo orientador, que altera a regra após 6 acertos consecutivos de uma mesma categoria. Não há tempo limite para a execução da prova. Este teste avalia funções executivas relacionadas à flexibilidade mental, categorização e perseveração.

- Compreensão: subteste da Escala de Inteligência Wechsler para adultos (WAIS-III) (Wechsler 1981, Wechsler 1997, Nascimento, 2004)

Neste subteste, o examinando deve responder, oralmente, a uma série de questões (totalizando 18) que exigem soluções para problemas cotidianos ou o entendimento de regras e conceitos sociais. Os escores variam de 0 a 2 pontos, para 5 dentre as 18 questões, e de 0 a 1 ponto para as demais questões, com a maior pontuação indicando a melhor resposta. Este teste avalia linguagem (compreensão) e funções executivas relacionadas à resolução de problemas.

- Teste da Figura de Rey (Rey, 1999; Spreen et al., 2006)

O teste consiste na cópia de uma figura geométrica complexa. Para o escore, são avaliados 18 aspectos, que podem pontuados como 0,1 (adequada precisão ou adequada localização) ou 2 (precisão e localização adequadas). A figura deve ser novamente 
reproduzida sem o auxílio do modelo e sem aviso prévio, após 30 minutos (evocação). Este teste avalia praxia construtiva e funções executivas relacionadas ao planejamento na cópia; e memória episódica visual, evocação.

- Teste de Aprendizagem Verbal Auditiva de Rey (RAVLT) (Lezak, 2004; Malloy-Diniz et al., 2007). O teste consiste na leitura de uma lista de 15 palavras. Após a leitura solicita-se ao sujeito, a evocação das palavras. Este procedimento é repetido cinco vezes. Anotam-se o número de palavras evocadas em cada tentativa. Uma nova lista de 15 palavras é lida e após a evocação desta segunda lista, solicita-se novamente a evocação da primeira. Após intervalo de tempo de 30 minutos, a evocação da primeira lista é novamente solicitada. Este teste avalia memória imediata, memória episódica e capacidade de aprendizagem.

- Memória Lógica (ML): subteste da Escala de Memória de Wechsler (WMS-R) (Wechsler,1987). Este teste é composto por duas estórias curtas, lidas em voz alta pelo examinador. O examinando é solicitado e relatar o que se lembra, imediatamente após a leitura de cada estória (etapa 1). Os itens recordados são anotados. Após 30 minutos, solicita-se a evocação tardia das estórias e novamente anotam-se os itens recordados (etapa 2) . O escore máximo de cada fase (evocação imediata e tardia) é de 50 pontos. Avalia memória imediata (etapa 1) e memória episódica tardia (etapa 2). 


\subsubsection{Avaliação do Julgamento}

- Test of Practical Judgment (TOP-J) (Rabin et al., 2007)

O TOP-J/15 foi gentilmente cedido pela autora, Dra. Laura Rabin, que deu permissão para a realização desde estudo. A versão original foi adaptada para uso no Brasil. A partir do TOP-J/15-Br foi desenvolvida uma versão brasileira de 9 itens (TOPJ/9-Br), conforme foi realizado pelos autores no TOP-J original (Rabin et al., 2007).

\subsection{Adaptação:}

\subsubsection{Processo de Tradução}

No processo de adaptação, foram seguidos os procedimentos: tradução, retro tradução, equivalência entre tradução e retro tradução, teste piloto e críticas finais por especialistas na área, baseados nas diretrizes elaboradas por Guillemin et al. (1993).

1) Foi realizada a tradução do inglês para o português por dois pesquisadores responsáveis, de forma independente;

2) A seguir as duas traduções foram comparadas e algumas adaptações foram realizadas em itens sem correspondência cultural ou funcional em nosso meio. Nestas adaptações, foram consideradas quatro tipos de equivalências importantes: semântica (analisa se as palavras têm o mesmo significado), idiomática (avalia eventuais coloquialismos), cultural (avalia se as experiências diárias podem ser traduzidas da mesma forma em diferentes culturas) e conceitual (avalia se o construto permaneceu o mesmo do original). As adaptações realizadas resultaram em uma versão de consenso 
entre os pesquisadores (Anexo E), na qual modificações foram realizadas nos itens 1, 2, $4,8,11,14$ e 15 .

3) Em seguida, foi realizado um estudo piloto com esta versão de consenso, que foi aplicada em 10 sujeitos sem queixas cognitivas, para avaliação da compreensão das questões, e da adequação dos itens. Outra modificação foi realizada, abrangendo os itens 1, 4, 13 e 15 (Anexo F).

4) Esta versão obtida após o estudo piloto foi submetida à retrotradução (Anexo G) por indivíduo com fluência na língua inglesa, e comparada à versão original.

5) A partir da discussão dos itens que apresentaram dificuldade de entendimento pela população avaliada, procedeu-se a uma terceira adaptação onde com poucas alterações foram realizadas nos itens $4,7,8,10,12$ e 15, para suprir as dificuldades encontradas, de forma a tornar inteligíveis as versões brasileiras finais, de 15 itens (TOPJ/15-Br) (Anexo H) e de 9 itens (TOP-J/9-Br) (Anexo I).

\subsubsection{Verificação da acurácia}

Para a verificação da acurácia, análises foram realizadas para verificação da validade, confiabilidade, sensibilidade e especificidade do TOP-J para a população brasileira.

Segundo Urbina (2007), os passos exigidos no processo de validação de um instrumento psicológico são diversos e não há na literatura consenso sobre quais e quantas evidências de validade o instrumento deve possuir para ser considerado válido. Neste estudo, foram investigadas a validade de conteúdo, a validade de critério e a validade de construto, processos mais investigados em trabalhos de adaptação (Pasquali, 2007), além da validade baseada na estrutura interna. 
A validade de conteúdo foi verificada na retro tradução, quando o instrumento foi vertido para o idioma original, visando testar se os itens traduzidos refletiriam o conteúdo da versão original.

A validade baseada na estrutura interna do TOP-J foi verificada através da análise fatorial exploratória.

A validade de critério do TOP-J foi verificada por meio da comparação dos resultados obtidos entre cada um dos grupos (controle, CCL, DA e DFT).

A validade por constructo relacionado do TOP-J foi observada pela associação dos seus resultados com medidas presumivelmente relacionadas (convergente) e não relacionadas (divergente).

A confiabilidade do TOP-J foi calculada através da consistência interna (Alpha de Cronbach), visto que este é um dos cálculos mais comuns para a análise da confiabilidade (Cardoso, 2006).

A sensibilidade e especificidade do TOP-J foram observadas através da curva ROC, identificação de pontos de corte que melhor possibilitassem distinguir os indivíduos controles de pacientes com CCL, controles de pacientes com demência (DA e DFT) e controles de todos os pacientes (CCL e DA e DFT).

As principais análises realizadas com a versão brasileira do TOP-J foram adotadas com base no estudo da versão original do TOP-J (Rabin et al., 2007).

\subsection{Análises estatísticas:}

A análise descritiva, também conhecida como análise exploratória, tem por objetivo conhecer o comportamento dos dados. Neste estudo, o comportamento dos dados foi conhecido a partir de uma análise descritiva com o cálculo da média, desvio 
padrão e valores mínimo e máximo para as variáveis quantitativas, juntamente com o teste de comparação de médias entre os grupos.

A parte inferencial do trabalho iniciou-se com os testes que verificam a suposição de normalidade, sendo eles o teste de Shapiro-Wilk, de Kolmogorov-Smirnov, de Cramer-Von Mises e de Anderson Darlin, nos quais a suposição de normalidade não foi atendida. Assim, os testes utilizados para comparação de médias foram não-paramétricos.

O teste não paramétrico de Qui Quadrado foi utilizado para avaliar a associação existente entre variáveis qualitativas.

O índice KMO (Kaiser's Measure of Sampling Adequacy) e o teste de esfericidade de Bartlett foram utilizados para verificar se a análise fatorial é pertinente aos dados. $\mathrm{O}$ KMO é um índice que varia entre 0 e 1 e é utilizado para verificar se a análise fatorial é adequada. Valores acima de 0,5 indicam que a análise é pertinente. De forma semelhante, o teste de esfericidade de Bartlett testa a hipótese de que as variáveis não sejam correlacionadas na população, em que sua hipótese básica diz que a matriz de correlação da população é uma matriz identidade, então, se o p-valor for menor que 0,05 , indica que o modelo fatorial é apropriado. A Análise Fatorial foi utilizada para verificar a possibilidade de retirada de itens do teste TOP-J, com o intuito de reduzir a dimensão do questionário e forneceu evidências de validade baseada na estrutura interna.

Para verificar se a média dos grupos era igual, o teste utilizado foi o de KruskallWallis. Quando o p-valor foi menor que 0,05 , indicando que existia pelo menos uma diferença entre as médias dos grupos, o teste de Bonferroni, de comparação de médias dois a dois foi realizado. Esta análise forneceu evidências da validade relativa ao critério. 
O coeficiente de correlação de Pearson foi utilizado para verificar a existência de relação linear entre variáveis quantitativas (Bussab e Moretin, 2002). Esta análise forneceu evidências de validade por constructo relacionado.

A análise de consistência interna do questionário será feita utilizando o Coeficiente de Alpha de Crombach, a fim de verificar se os itens medem o mesmo constructo e, assim, verificar se são altamente intercorrelacionados. O coeficiente Alpha de Cronbach varia entre 0 e 1 (Hair et al., 1998). Esta análise ofereceu evidências de confiabilidade.

A sensibilidade e especificidade do questionário foram avaliadas por meio de curvas ROC (Receiving Operator Characteristic Curve). A curva é construída locando-se a taxa de verdadeiros-positivos (sensibilidade) contra a taxa de falsos-positivos (1especificidade), ao longo de uma faixa de pontos de corte. Os valores nos eixos vão de uma chance de 0 a 100\% (Fletcher e Fletcher, 2006). A curva ROC representa os pontos de corte possíveis e mostra a área sob a curva. Foram escolhidos pontos de corte com a maior sensibilidade e com a maior especificidade possível (que não abaixasse o valor da sensibilidade).

Para análise estatística foi utilizado o programa computacional SPSS v.19.0. O nível de significância adotado para os testes estatísticos foi de 5\%, ou seja, $p$-valor $<0,05$. 


\section{RESULTADOS}

Os resultados serão apresentados separadamente, na seguinte ordem: análise descritiva de caracterização da amostra, análise descritiva dos resultados dos testes para cada grupo, análise das evidências de validade baseada na estrutura interna e redução da escala, análise das evidências de validade relativa ao critério, análise das evidências de validade por constructo relacionado, análise das evidências de confiabilidade, e análise da sensibilidade e especificidade, para o TOP-J/15-Br e TOP-J/9-Br, respectivamente.

\subsection{Análise Descritiva de Caracterização da Amostra}

$\mathrm{Na}$ tabela 3 são apresentadas as características sociodemográficas dos participantes e resultados dos testes utilizados para caracterização diagnóstica. Observase que os grupos foram homogêneos em relação ao gênero e à escolaridade, mas não em relação à idade (controle $<$ CCL e DA, DFT < CCL e DA). 
Tabela 3: Perfil sociodemográfico dos participantes

\begin{tabular}{|c|c|c|c|c|c|c|c|c|c|c|c|c|}
\hline \multirow{3}{*}{ Variável } & & \multicolumn{11}{|c|}{ Grupos } \\
\hline & & \multicolumn{2}{|c|}{ Total } & \multicolumn{2}{|c|}{ Controle } & \multicolumn{2}{|c|}{ CCL } & \multicolumn{2}{|c|}{ DA } & \multicolumn{2}{|c|}{ DFT } & \multirow[t]{2}{*}{ p-valor } \\
\hline & & $\mathrm{N}=85$ & $\%$ & $\mathrm{~N}=24$ & $\%$ & $\mathrm{~N}=26$ & $\%$ & $\mathrm{~N}=20$ & $\%$ & $\mathrm{~N}=15$ & $\%$ & \\
\hline \multicolumn{13}{|l|}{ Gênero } \\
\hline & Feminino & 39 & 45,88 & 8 & 33,3 & 10 & 38,5 & 9 & 45 & 12 & 80 & \multirow{2}{*}{$* * * 0,45$} \\
\hline & Masculino & 46 & 54.12 & 16 & 66,7 & 16 & 61,5 & 11 & 55 & 3 & 20 & \\
\hline \multirow[t]{3}{*}{ Idade } & Média (DP) & 69,6 & 7,8 & 65,5 & 7,05 & 71,6 & 5,52 & 75,1 & 6,24 & 65,3 & 8,68 & \multirow{3}{*}{$* * 0,00^{\mathrm{A}, \mathrm{B}, \mathrm{E}, \mathrm{F}}$} \\
\hline & Mediana & \multicolumn{2}{|c|}{70} & \multicolumn{2}{|c|}{65} & \multicolumn{2}{|c|}{72} & \multicolumn{2}{|c|}{77} & \multicolumn{2}{|c|}{67} & \\
\hline & Mínimo - Máximo & 51 & 84 & 51 & 81 & 60 & 81 & 58 & 84 & 51 & 79 & \\
\hline \multicolumn{13}{|c|}{ Escolaridade } \\
\hline & Média (DP) & 10,7 & 5,4 & 12,3 & 3,05 & 10,4 & 6,51 & 9,3 & 5,19 & 10,3 & 6,08 & \\
\hline & Mediana & \multicolumn{2}{|c|}{11} & \multicolumn{2}{|c|}{13} & \multicolumn{2}{|c|}{9} & \multicolumn{2}{|c|}{8} & \multicolumn{2}{|c|}{8} & \multirow[t]{2}{*}{$* 0,12$} \\
\hline & Mínimo - Máximo & 4 & 35 & 4 & 16 & 4 & 35 & 4 & 20 & 4 & 20 & \\
\hline \multirow[t]{5}{*}{ Testes } & Média (DP) & & & & & & & & & & & \\
\hline & MEEM & & & 29,3 & 0,7 & 27,46 & 1,5 & 24,1 & 2,7 & 24,67 & 3,6 & $* * 0,0001^{\mathrm{A}, \mathrm{B}, \mathrm{C}, \mathrm{D}, \mathrm{E}}$ \\
\hline & BBRC & & & 9 & 1,2 & 7,31 & 1,67 & 3,1 & 2,2 & 5,67 & 3,33 & $* * 0,0001^{\mathrm{A}, \mathrm{B}, \mathrm{C}, \mathrm{D}, \mathrm{E}, \mathrm{F}}$ \\
\hline & QAF & & & 0,3 & 0,6 & 1,62 & 1,6 & 8,3 & 6 & 13 & 7,08 & $* * 0,0001^{\mathrm{A}, \mathrm{B}, \mathrm{C}, \mathrm{D}, \mathrm{E}, \mathrm{F}}$ \\
\hline & IQCODE & & & 2,5 & 0,8 & 3,04 & 0,74 & 3,7 & 0,9 & 3,73 & 0,79 & $* * 0,0001^{\mathrm{A}, \mathrm{B}, \mathrm{C}, \mathrm{D}, \mathrm{E}}$ \\
\hline
\end{tabular}

\subsection{Análise Descritiva dos Resultados dos Testes}

$\mathrm{Na}$ tabela 4, serão apresentadas as análises descritivas dos testes utilizados para os grupos controle, CCL, DA e DFT, bem como os p-valores segundo o teste de comparação de médias Kruskal-Wallis, em que, caso o p-valor seja menor que 0,05 , existe pelo menos uma diferença entre as médias dos grupos, e para verificar quais grupos tem médias distintas, o teste-t de Bonferroni foi realizado fazendo uma comparação 2 a 2 entre os grupos, também determinada quando o p-valor é menor que 0,05 .

Observou-se que, comparando-se controles aos pacientes com CCL, houve diferença estatisticamente significativa em medidas de memória verbal e não verbal e funções executivas.

Entre controles e pacientes com DA, houve diferença estatisticamente significativa em todos os testes, exceto na extensão de dígitos em ordem direta. 
Comparando-se controles aos pacientes com DFT, houve diferença estatisticamente significativa em todos os testes.

Comparando-se pacientes com CCL a pacientes com DA, houve diferença estatisticamente significativa em fluência verbal por categoria semântica e fonêmica, medidas de memória verbal e não verbal, funções executivas e Compreensão.

Comparando-se pacientes com CCL a pacientes com DFT, houve diferença estatisticamente significativa em fluência semântica e fonêmica, funções executivas, memória verbal e não verbal e atenção.

Comparando-se pacientes com DA a pacientes com DFT, houve diferença estatisticamente significativa em memória verbal (memória lógica) e atenção.

Tabela 4: Comparação do desempenho entre os grupos nos testes aplicados.

\begin{tabular}{|c|c|c|c|c|c|c|c|c|c|}
\hline \multirow[t]{2}{*}{ Variáveis } & \multicolumn{2}{|c|}{ Controle } & \multicolumn{2}{|c|}{$\mathrm{CCL}$} & \multicolumn{2}{|c|}{$\mathrm{DA}$} & \multicolumn{2}{|c|}{ DFT } & \multirow[b]{2}{*}{ p-valor* } \\
\hline & Média & $\mathrm{DP} \pm$ & Média & $\mathrm{DP} \pm$ & Média & $\mathrm{DP} \pm$ & Média & $\mathrm{DP} \pm$ & \\
\hline FV (animais) & 16,8 & 4 & 15,04 & 4,28 & 11,1 & 2,9 & 11,2 & 4,57 & $0,0001^{\mathrm{B}, \mathrm{C}, \mathrm{D}, \mathrm{E}}$ \\
\hline FAS & 32,4 & 9,7 & 29,92 & 8,29 & 23,4 & 7,5 & 19,87 & 10,35 & $0,0005^{\mathrm{B}, \mathrm{C}, \mathrm{D}, \mathrm{E}}$ \\
\hline DR & 8,8 & 1,1 & 8,31 & 1,72 & 7,4 & 1,6 & 6,4 & 2,23 & $0,0001^{\mathrm{B}, \mathrm{C}, \mathrm{E}}$ \\
\hline ML1 & 24,7 & 5,7 & 19,15 & 6,87 & 8,7 & 6,3 & 14,8 & 8,95 & 0,0001 A, B, C, D, F \\
\hline ML2 & 21,2 & 5,6 & 12,58 & 8,64 & 2,3 & 4,3 & 8,53 & 6,56 & $0,0001 \mathrm{~A}, \mathrm{~B}, \mathrm{C}, \mathrm{D}, \mathrm{F}$ \\
\hline RAVLT - T & 44,1 & 8,6 & 33,96 & 9,13 & 22,6 & 7,7 & 24,53 & 7,9 & 0,0001 A, B, C, D, E \\
\hline RAVLT - 30' & 8,7 & 2,8 & 5,81 & 2,9 & 1,1 & 1,4 & 2,87 & 2,9 & 0,0001 A, B, C, D, E, F \\
\hline Figura Rey - C & 27,9 & 5,2 & 27,27 & 5,25 & 17,8 & 9 & 16,77 & 9,63 & $0,0001^{\mathrm{B}, \mathrm{C}, \mathrm{D}, \mathrm{E}}$ \\
\hline Figura Rey -E & 10,6 & 6 & 6,69 & 4,97 & 1,8 & 2,5 & 3,13 & 3,45 & $0,0001 \mathrm{~A}, \mathrm{~B}, \mathrm{C}, \mathrm{D}, \mathrm{E}$ \\
\hline TMTA-T & 37,6 & 12,1 & 54,12 & 23,78 & 77,3 & 32 & 66,6 & 39,06 & 0,0001 A, B, C, D \\
\hline TMTB-T & 85,7 & 34,4 & 168,5 & 79,19 & 215,6 & 106,6 & 201,2 & 93,1 & $0,0001^{\mathrm{A}, \mathrm{B}, \mathrm{C}}$ \\
\hline OD & 7,5 & 2,4 & 7,15 & 1,59 & 6,8 & 1,4 & 5,73 & 1,16 & $0,0217^{\mathrm{C}, \mathrm{E}, \mathrm{F}}$ \\
\hline OI & 5 & 1,6 & 4,88 & 1,56 & 3,7 & 1,3 & 3,13 & 1,25 & $0,0003^{\mathrm{B}, \mathrm{C}, \mathrm{D}, \mathrm{E}}$ \\
\hline WCST & 2,7 & 1,4 & 2,19 & 1,06 & 1,9 & 0,8 & 1,27 & 0,96 & $0,0064^{\text {B, C, E, F }}$ \\
\hline $\mathrm{MZ}$ & 1,9 & 0,8 & 1,46 & 0,86 & 1,3 & 1 & 1,2 & 0,77 & $0,0516^{\mathrm{B}, \mathrm{C}}$ \\
\hline Compreensão & 20,7 & 3,6 & 17,12 & 6,04 & 13 & 4,9 & 14,33 & 6,15 & 0,0001 A, B, C, D \\
\hline $\begin{array}{l}\text { genda: GDS: Geriatri } \\
\text { nêmicas (letras F, A e } \\
\text { ey Auditory Verbal Le } \\
\text { rdia); Figura Rey - C } \\
\text { rte A (tempo); TMT-B } \\
\text { rdim Zoológico; A= C } \\
\text { uskal-Wallis seguido }\end{array}$ & Depressior & S Scale; $\mathrm{F}$ & $\mathrm{L}$ (animais & : fluência & verbal s & antica ca & tegoria an & ais); FAS & $\begin{array}{l}\text { luência Verbal para catego } \\
\text { Lógica } 2 \text { (tardia); RAVL } \\
\text { Learning Test após } 30 \text { min } \\
\text { de Trilhas (Trail Making T } \\
\text { g Test; MZ: Teste do Mapa } \\
\text { FT; F=DA \& DFT. * Test }\end{array}$ \\
\hline
\end{tabular}




\subsection{Validade}

\subsubsection{Validade Baseada na Estrutura Interna}

$\mathrm{O}$ índice de Kaiser-Meyer-Olkin geral $(\mathrm{KMO}=0,64 ;>0,5)$ e o teste de esfericidade de Bartlett ( $\mathrm{p}<0,05$; estatística de teste 179,63 e grau de liberdade 105) indicaram que a análise fatorial é pertinente aos dados e que a amostra estava adequada para a realização da análise fatorial exploratória.

\subsubsection{Redução da Escala}

Para reduzir a dimensão do TOP-J/15-Br, a análise fatorial foi realizada e os resultados são apresentados na tabela 5. Foram identificados cinco fatores que explicariam ao menos $54 \%$ da variabilidade dos dados. Os fatores tiveram autovalores maiores que 1 (mais especificamente entre 1,07 e 2,98).

Tomando por base o artigo do TOP-J original (Rabin et al., 2007), apenas o primeiro fator foi considerado para identificar as cargas fatoriais menores que 0,4 . Desta forma, seis itens puderam ser excluídos, sendo eles: 3, 5, 9, 11, 13 e 15 . Então, a versão reduzida será composta pelos nove itens restantes $(1,2,4,6,7,8,10,12$ e 14).

Os itens 4, 6, 7, 8, 10, 12 e 14 foram posteriormente reordenados como 3, 4, 5, 6, 7, 8 e 9. Os itens mantidos no TOP-J/9 abrangeram todos os domínios propostos pelos autores, dentre eles dois de domínio médico (itens 1 e 4); dois de domínio financeiro (itens 2 e 3), quatro de domínio social/ético (itens 6, 7, 8 e 9), e um de domínio segurança (item 5). Os itens mantidos no TOP-J/9 original (Rabin et al., 2007) não foram exatamente os mesmos mantidos no TOP-J/9-Br. Na versão original (Rabin et al., 2007), após a extração de um único fator no TOP-J 15, foram mantidos os itens 1, 2, 3, 4, 6, 7, 8, 12 e 15 . Os itens numerados como 6, 7, 8, 12 e 15 foram subsequentemente reordenados 
como 5, 6, 7, 8 e 9 na versão final de 9 itens. Os itens mantidos no TOP-J/9 original abrangem todos os domínios propostos para o de 15 itens, sendo dois itens do domínio médico (itens 1 e 5), três relacionados ao domínio financeiro (itens 2 , 4 e 9), dois relacionados ao domínio social ético (itens 7 e 8 ) e dois relacionados ao domínio segurança (itens 3 e 6). O TOP-J/9-Br e o TOP-J/9 original têm em comum 7 itens (itens 1, 2, 4, 6, 7, 8 e 12 do TOP-J/15). A tabela 5 ilustra essas informações.

Tabela 5. Comparação entre o TOP-J/9 original e o TOP-9/Br a partir do TOP-J/15

\begin{tabular}{llcccc}
\hline Item & Domínio & TOP-J/9 original & $\begin{array}{l}\text { TOP-J/9 original } \\
\text { reordenado }\end{array}$ & TOP-J/9-Br & $\begin{array}{c}\text { TOP-J/9-Br } \\
\text { reordenado }\end{array}$ \\
\hline $\mathbf{1}$ & Médico & 1 & 1 & 1 & 1 \\
$\mathbf{2}$ & Financeiro & 2 & 2 & 2 & 2 \\
3 & Segurança & 3 & 3 & 4 & 3 \\
$\mathbf{4}$ & Financeiro & 4 & 4 & 6 & 4 \\
5 & Social & & & 7 & 5 \\
$\mathbf{6}$ & Médico & 6 & 6 & 8 & 6 \\
$\mathbf{7}$ & Segurança & 7 & 7 & 10 & 7 \\
$\mathbf{8}$ & Social & 8 & & 12 & \\
9 & Segurança & & & & \\
10 & Social & & & & \\
11 & Segurança & & & & \\
12 & Social & 12 & 9 & & \\
13 & Médico & & & &
\end{tabular}

No TOP-J/9-Br, a análise fatorial também foi pertinente, mesmo com a exclusão dos 6 itens, uma vez que o índice de KMO Geral é de 0,72 (maior que 0,5) e que o teste de esfericidade de Bartlett tem p-valor 0,00, que é menor que 0,05 (com estatística de teste 97,78 e grau de liberdade 36). Então, conforme realizado no estudo original (Rabin et al., 2007), outra análise fatorial exploratória para um único fator foi realizada com os 9 itens restantes. As cargas fatoriais de cada um dos itens sobre o fator geral, para o TOPJ/15-Br e TOP-J/9-Br são mostradas na tabela 6.

Observa-se também a comparação entre as cargas fatoriais com base na análise fatorial exploratória para um único fator, para o TOP-J/9 original e TOP-J/9-Br. 
Tabela 6. Análise fatorial exploratória para um único fator

\begin{tabular}{ccccc} 
& Domínio & $\begin{array}{c}\text { Cargas fatoriais do } \\
\text { TOP-J/15-Br }\end{array}$ & $\begin{array}{c}\text { Cargas fatoriais do } \\
\text { TOP-J/9-Br }\end{array}$ & $\begin{array}{c}\text { Cargas fatoriais do } \\
\text { TOP-J/9 original }\end{array}$ \\
\hline Item & & $\mathbf{0 , 4 3}$ & $\mathbf{0 , 3 6}$ & $\mathbf{0 , 6 2}$ \\
TOP-J 1 & Médico & $\mathbf{0 , 4 9}$ & $\mathbf{0 , 5 7}$ & $\mathbf{0 , 4 3}$ \\
TOP-J 2 & Financeiro & $0,23^{*}$ & $\mathrm{n} / \mathrm{a}^{*}$ & 1,10 \\
TOP-J 3* & Segurança* & $\mathbf{0 , 5 1}$ & $\mathbf{0 , 5 5}$ & $\mathbf{0 , 6 0}$ \\
TOP-J 4 & Financeiro & $0,39^{*}$ & $\mathrm{n} / \mathrm{a}^{*}$ & $\mathrm{n} / \mathrm{a}$ \\
TOP-J 5* & Social* & $\mathbf{0 , 7 7}$ & $\mathbf{0 , 7 9}$ & $\mathbf{0 , 6 8}$ \\
TOP-J 6 & Médico & $\mathbf{0 , 4 3}$ & $\mathbf{0 , 4 6}$ & $\mathbf{0 , 4 5}$ \\
TOP-J 7 & Segurança & $\mathbf{0 , 4 9}$ & $\mathbf{0 , 3 7}$ \\
TOP-J 8 & Social & $0,38^{*}$ & $\mathrm{n} / \mathrm{a}$ & $\mathrm{n} / \mathrm{a}$ \\
TOP-J 9* & Segurança* & 0,50 & 0,54 & $\mathrm{n} / \mathrm{a}$ \\
TOP-J 10 & Social & $0,34^{*}$ & $\mathrm{n} / \mathrm{a}^{*}$ & $\mathrm{n} / \mathrm{a}$ \\
TOP-J 11* & Segurança* & $\mathbf{0 , 3 1}$ & $\mathbf{0 , 4 1}$ \\
TOP-J 12 & Social & $0,15^{*}$ & $\mathrm{n} / \mathrm{a}^{*}$ & $\mathrm{n} / \mathrm{a}$ \\
TOP-J 13* & Médico* & 0,53 & 0,58 & $\mathrm{n} / \mathrm{a}$ \\
TOP-J 14 & Social & $0,33^{*}$ & $\mathrm{n} / \mathrm{a}^{*}$ & 0,49 \\
TOP-J 15* & Financeiro* & & & \\
\hline Nota: Itens* não constam no TOP-J/9 Brasil; Itens em negrito são comuns ao TOP-J/9 original e ao TOP-J/9 Brasil; Os itens estão \\
numerados de acordo com a posição no protocolo do TOP-J/15.
\end{tabular}

Essa redução da escala forneceu uma diminuição de tempo de aplicação. Em geral, no teste com 15 itens eram gastos em média 15 minutos e com a versão reduzida, aproximadamente 10 minutos.

\subsubsection{Validade Relativa ao Critério}

A tabela 7 mostra que no escore total do TOP-J/15-Br e do TOP-J/9-Br houve diferença estatisticamente significativa entre Controle e CCL, Controle e DA, Controle e DFT, CCL e DA, e CCL e DFT. Não houve diferença estatisticamente significativa entre DA e DFT. 
Tabela 7. Comparação do desempenho entre os grupos no TOP-J

\begin{tabular}{|c|c|c|c|c|c|c|c|c|c|}
\hline \multirow[t]{2}{*}{ Variáveis } & \multicolumn{2}{|c|}{ Controle } & \multicolumn{2}{|c|}{$\mathrm{CCL}$} & \multicolumn{2}{|c|}{ DA } & \multicolumn{2}{|c|}{ DFT } & \multirow{2}{*}{$\begin{array}{l}\text { Comparação de } \\
\text { médias }\end{array}$} \\
\hline & Média & $\mathrm{DP} \pm$ & Média & $\mathrm{DP} \pm$ & Média & $\mathrm{DP} \pm$ & Média & $\mathrm{DP} \pm$ & \\
\hline TOP-J1 & 2,00 & 1,10 & 1,62 & 1,33 & 1,40 & 1,27 & 1,00 & 1,25 & 0,0973 \\
\hline TOP-J2 & 2,00 & 0,29 & 1,88 & 0,52 & 1,75 & 0,72 & 1,40 & 0,91 & 0,0712 \\
\hline TOP-J3 & 2,88 & 0,61 & 2,69 & 0,84 & 2,45 & 1,00 & 2,47 & 0,99 & 0,2129 \\
\hline TOP-J4 & 1,88 & 1,08 & 1,54 & 1,10 & 1,10 & 1,07 & 0,53 & 0,92 & $0,0019^{\mathrm{B}, \mathrm{C}, \mathrm{E}}$ \\
\hline TOP-J5 & 2,50 & 0,78 & 2,19 & 0,85 & 2,15 & 0,67 & 1,67 & 1,11 & $0,0568^{\mathrm{C}}$ \\
\hline TOP-J6 & 2,92 & 0,41 & 2,38 & 0,94 & 2,20 & 1,01 & 1,60 & 1,12 & $0,0005^{\mathrm{A}, \mathrm{B}, \mathrm{C}, \mathrm{E}}$ \\
\hline TOP-J7 & 2,08 & 0,93 & 1,81 & 0,94 & 1,35 & 0,59 & 1,53 & 0,74 & $0,0477^{\mathrm{B}}$ \\
\hline TOP-J8 & 2,08 & 0,58 & 1,65 & 0,63 & 1,30 & 1,13 & 1,47 & 0,83 & $0,0312^{\mathrm{A}, \mathrm{B}, \mathrm{C}}$ \\
\hline TOP-J9 & 2,00 & 1,32 & 1,92 & 1,09 & 2,15 & 1,18 & 1,73 & 1,33 & 0,6460 \\
\hline TOP-J10 & 2,79 & 0,59 & 2,58 & 0,76 & 2,35 & 0,81 & 2,20 & 1,26 & 0,1018 \\
\hline TOP-J11 & 2,96 & 0,20 & 2,81 & 0,69 & 2,50 & 1,00 & 2,47 & 1,13 & 0,1364 \\
\hline TOP-J12 & 1,42 & 1,10 & 1,46 & 1,10 & 0,80 & 0,77 & 1,07 & 1,10 & 0,1349 \\
\hline TOP-J13 & 1,58 & 0,58 & 1,46 & 0,65 & 1,50 & 0,95 & 1,53 & 0,74 & 0,8495 \\
\hline TOP-J14 & 3,00 & 0,00 & 2,81 & 0,69 & 2,55 & 0,89 & 2,47 & 1,13 & $0,0551^{\mathrm{B}, \mathrm{C}}$ \\
\hline TOP-J15 & 1,38 & 0,97 & 1,42 & 0,86 & 0,95 & 0,76 & 1,07 & 0,80 & 0,2185 \\
\hline TOP-J/15-Br & 33,4 & 4 & 30,15 & 4,88 & 26,5 & 4,9 & 24,2 & 6,47 & $0,0001^{\text {A, B, C, D, E }}$ \\
\hline TOP-J/9-Br & 20,2 & 2,4 & 17,7 & 3,5 & 14,8 & 4,0 & 13,3 & 4,9 & $0,0001^{\text {A, B, C, D, E }}$ \\
\hline
\end{tabular}

*Teste de Kruskal-Wallis seguido do teste de Bonferroni: A) Controle $\neq \mathrm{CCL}$; B) Controle $\neq \mathrm{DA}$; C) Controle $\neq \mathrm{DFT}$; D) CCL $\neq \mathrm{DA}$; E) $\mathrm{CCL} \neq \mathrm{DFT}$; F) DA $\neq$ DFT

\subsubsection{Validade por Constructo Relacionado}

Para a verificação da validade por constructo relacionado, investigou-se as associações entre TOP-J e medidas de habilidades relacionadas (validade convergente) e não relacionadas (validade divergente) à habilidade de julgamento, no grupo controle.

Para analisar evidências de validade convergente, observamos a correlação entre o TOP-J/15-Br e o TOP-J/9-Br e o teste Compreensão, por avaliar habilidades relacionadas, como abstração verbal, julgamento moral, planejamento, entendimento de regras sociais, e por ser frequentemente utilizado por neuropsicólogos como medida de avaliação de julgamento (Rabin et al., 2008). Para analisar evidências de validade divergente, observamos a correlação entre o TOP-J/15-Br e TOP-J/9-Br e medida de humor (GDS), pelo fato de o TOP-J ter menor envolvimento com funcionamento emocional. 
Houve correlação positiva do escore total do TOP-J/15-Br e do TOP-J/9-Br com o teste Compreensão, o que aponta para evidência de validade convergente. A correlação do TOP-J/15-Br com o MZ, teste que avalia planejamento e seguimento de normas (relacionadas a julgamento), apoia a evidência de validade convergente.

Não houve correlação entre o TOP-J/15-Br e do TOP-J/9-Br com a GDS, o que aponta para evidência de validade divergente. A tabela 8 mostra os valores das correlações entre o TOP-J/15 e TOP-J/9 e os demais testes, para o grupo controle.

Tabela 8: Correlação de Pearson entre TOP-J/15-Br e TOP-J/9-Br e demais testes.

\begin{tabular}{lcccc}
\hline \multicolumn{1}{c}{ Variáveis } & \multicolumn{2}{c}{ TOP-J/15-Br } & \multicolumn{2}{c}{ TOP-J/9-Br } \\
\hline GDS & Coeficiente (RHO) & p-valor & Coeficiente (RHO) & p-valor \\
ML1 & 0,21 & 0,32 & 0,25 & 0,24 \\
ML2 & 0,18 & 0,41 & 0,37 & 0,08 \\
RAVLT-T & 0,22 & 0,31 & 0,30 & 0,16 \\
RAVLT-30, & 0,17 & 0,43 & 0,16 & 0,45 \\
Figura Rey - C & 0,02 & 0,93 & 0,14 & 0,53 \\
Figura Rey-E & $-0,18$ & 0,39 & $-0,18$ & 0,41 \\
FAS & 0,01 & 0,95 & 0,00 & 0,99 \\
TMTA-T & 0,13 & 0,53 & 0,16 & 0,45 \\
TMTB-T & $-0,25$ & 0,23 & $-0,33$ & 0,12 \\
OD & $-0,28$ & 0,18 & $-0,35$ & 0,10 \\
OI & 0,36 & 0,09 & 0,25 & 0,25 \\
WCST & 0,28 & 0,18 & 0,10 & 0,64 \\
MZ & 0,25 & 0,24 & 0,25 & 0,23 \\
Compreensão & 0,40 & $\mathbf{0 , 0 5}$ & 0,27 & 0,21 \\
\hline
\end{tabular}

Legenda: GDS: Geriatric Depression Scale; FL (animais): fluência verbal semântica categoria animais); FAS: Fluência Verbal para categorias fonêmicas (letras F, A e S); DR: Teste desenho do relógio; ML1: Memória Lógica 1 (imediata); ML2: Memória Lógica 2 (tardia); RAVLT-T: Rey Auditory Verbal Learning Test Total (somatória evocações imediatas); RAVLT-30’: Rey Auditory Verbal Learning Test após 30 minutos (tardia); Figura Rey - C (Figura de Rey-Cópia); Figura Rey - E (Figura de Rey - Evocação); TMTA-T: Teste de Trilhas (Trail Making Test) parte A (tempo); TMTB-T: Teste de Trilhas (Trail Making Test) parte B (tempo); WCST: Winsconsin Card Sorting Test; MZ: Teste do Mapa do Jardim Zoológico.

\subsection{Confiabilidade}

\section{Consistência Interna}

Observou-se que o TOP-J/15-Br como um todo alcançou boa consistência interna (Alfa de Cronbach=0,69). No TOP-J/9-Br, a consistência interna foi mantida (Alfa de Cronbach $=0,68)$. A tabela 9 mostra a análise de consistência interna de todos os itens do TOP-J/15 e do TOP-J/9 
Tabela 9. Análise da Consistência Interna do TOP-J/15-Br e do TOP-J/9-Br

\begin{tabular}{|c|c|c|c|c|c|c|c|c|}
\hline \multirow[t]{2}{*}{ Itens } & \multicolumn{2}{|c|}{$\begin{array}{c}\text { Média após a retirada } \\
\text { dos itens }\end{array}$} & \multicolumn{2}{|c|}{$\begin{array}{l}\text { Desvio padrão após a } \\
\text { retirada dos itens }\end{array}$} & \multicolumn{2}{|c|}{$\begin{array}{l}\text { Correlação com o } \\
\text { total }\end{array}$} & \multicolumn{2}{|c|}{$\begin{array}{l}\text { Coeficiente* (após a } \\
\text { retirada dos itens) }\end{array}$} \\
\hline & TOP-J/15 & TOP-J/9 & TOP-J/15 & TOP-J/9 & TOP-J/15 & TOP-J/9 & TOP-15 & TOP-J/9 \\
\hline TOP-J 1 & 27,64 & 15,38 & 5,47 & 3,95 & 0,32 & 0,25 & 0,67 & 0,67 \\
\hline TOP-J 2 & 27,4 & 15,14 & 5,78 & 4,19 & 0,3 & 0,34 & 0,67 & 0,64 \\
\hline TOP-J 3 & 26,55 & & 5,83 & & 0,14 & & 0,69 & \\
\hline TOP-J 4 & 27,85 & 15,59 & 5,54 & 3,91 & 0,32 & 0,35 & 0,67 & 0,63 \\
\hline TOP-J 5 & 27,02 & & 5,69 & & 0,29 & & 0,67 & \\
\hline TOP-J 6 & 26,85 & 14,59 & 5,39 & 3,81 & 0,57 & 0,58 & 0,63 & 0,58 \\
\hline TOP-J 7 & 27,47 & 15,21 & 5,69 & 4,08 & 0,3 & 0,33 & 0,67 & 0,64 \\
\hline TOP-J 8 & 27,54 & 15,28 & 5,67 & 4,07 & 0,34 & 0,36 & 0,67 & 0,63 \\
\hline TOP-J 9 & 27,24 & & 5,56 & & 0,27 & & 0,68 & \\
\hline TOP-J 10 & 26,88 & 14,42 & 5,67 & 4,08 & 0,32 & 0,33 & 0,67 & 0,64 \\
\hline TOP-J 11 & 26,48 & & 5,76 & & 0,25 & & 0,68 & \\
\hline TOP-J 12 & 27,98 & 15,72 & 5,6 & 4,06 & 0,3 & 0,25 & 0,67 & 0,66 \\
\hline TOP-J 13 & 27,68 & & 5,88 & & 0,11 & & 0,69 & \\
\hline TOP-J 14 & 26,46 & 14,20 & 5,71 & 4,11 & 0,33 & 0,36 & 0,67 & 0,63 \\
\hline TOP-J 15 & 27,96 & & 5,72 & & 0,26 & & 0,67 & \\
\hline
\end{tabular}

*coeficiente alfa de Cronbach

\subsection{Sensibilidade e Especificidade}

A tabela 10 mostra que o TOP-J/15-Br e o TOP-J/9-Br apresentam boa acurácia para discriminar controles de pacientes com CCL, DA e DFT, controles de pacientes com demência (DA e DFT) e controles de pacientes (CCL, DA e DFT). Nela estão demonstrados os valores de sensibilidade, especificidade e área sob a curva (curva ROC). 
Tabela 10. Áreas sob a Curva, Pontos de Corte, Sensibilidade e Especificidade

\begin{tabular}{llcccc}
\hline Grupos & $\begin{array}{c}\text { Área sob a } \\
\text { Curva }\end{array}$ & $\begin{array}{c}\text { Ponto de } \\
\text { Corte }\end{array}$ & $\begin{array}{c}\text { Sensibilidade } \\
\%\end{array}$ & $\begin{array}{c}\text { Especificidade } \\
\%\end{array}$ \\
\hline TOP-J/15 & Controle versus CCL & $0,70^{* *}$ & 31 & 87,5 & 46,2 \\
& Controle versus DA & $0,87^{*}$ & 29 & 91,7 & 70,0 \\
& Controle versus DFT & $0,89^{*}$ & 30 & 91,7 & 73,3 \\
& Controle versus Demência & $0,88^{*}$ & 30 & 91,7 & 71,4 \\
& Controle versus Paciente & $0,80^{*}$ & 30 & 91,7 & 59,0 \\
\hline TOP-J/9 & Controle versus CCL & $0,73^{* *}$ & 19 & 79,2 & 57,7 \\
& Controle versus DA & $0,87^{*}$ & 18 & 83,3 & 70,0 \\
& Controle versus DFT & $0,90^{*}$ & 18 & 83,3 & 73,3 \\
& Controle versus Demência & $0,89^{*}$ & 18 & 83,3 & 71,4 \\
& Controle versus Paciente & $0,82^{*}$ & 19 & 79,2 & 72,1 \\
\hline
\end{tabular}

Nota: ${ }^{*}$ p-valor $<0,001 ; * *$ p-valor $<0,05$

As figuras 2 e 3 mostram as curvas ROC do TOP-J/15 $\mathrm{Br}$ e do TOP-J/9 $\mathrm{Br}$ respectivamente. 
Figura 2. Curvas ROC do TOP-J/15-Br

(a) Controle versus CCL

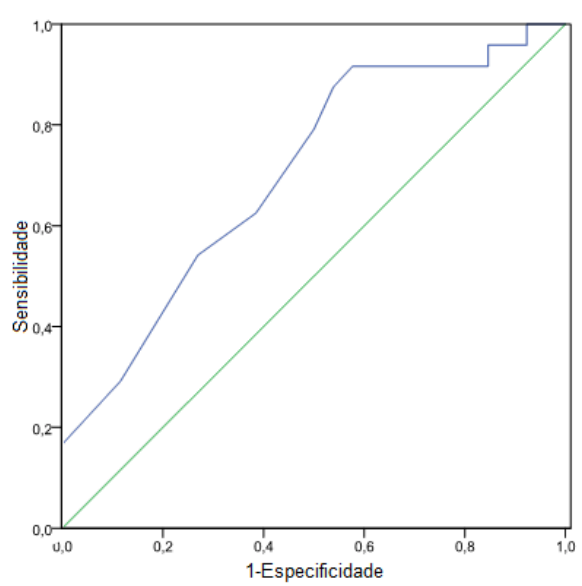

(c) Controle versus DFT

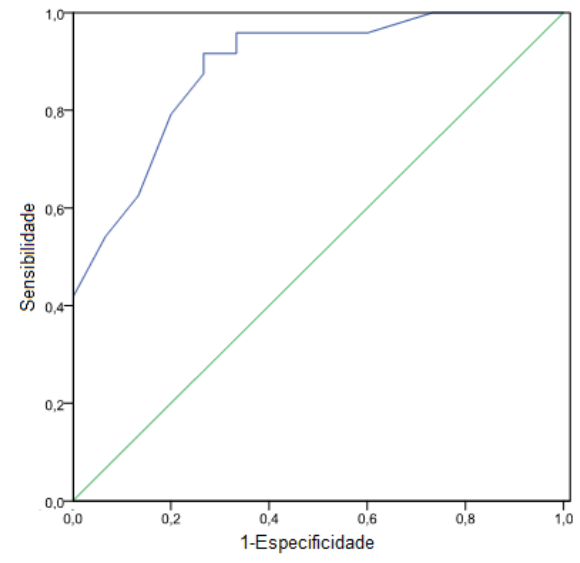

(b) Controle versus DA

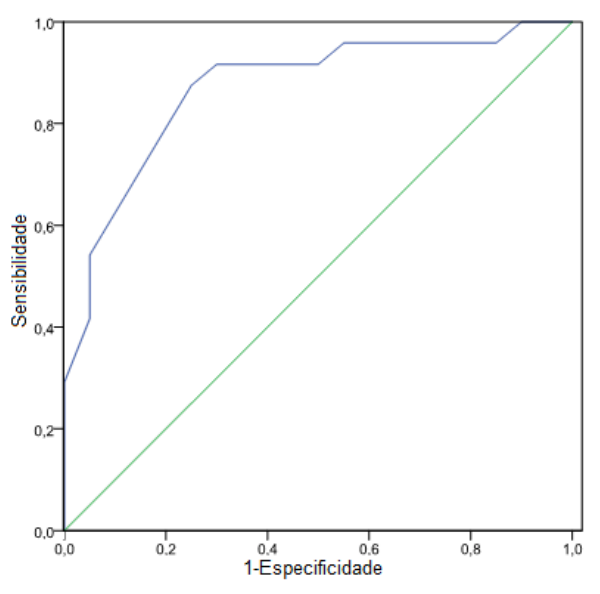

(d) Controle versus Demência

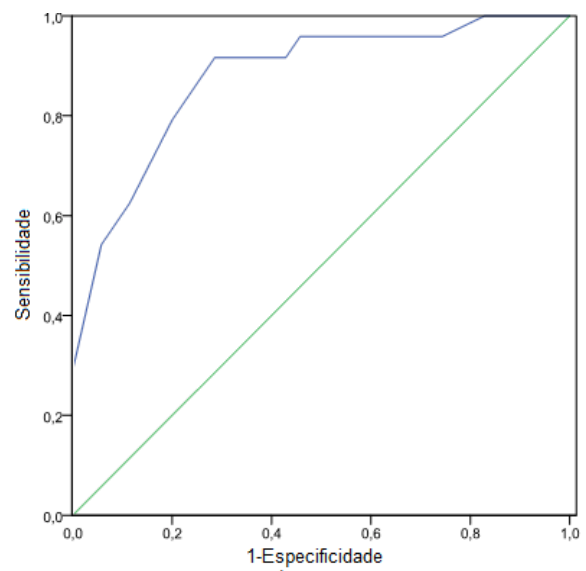

(e) Controle versus Paciente

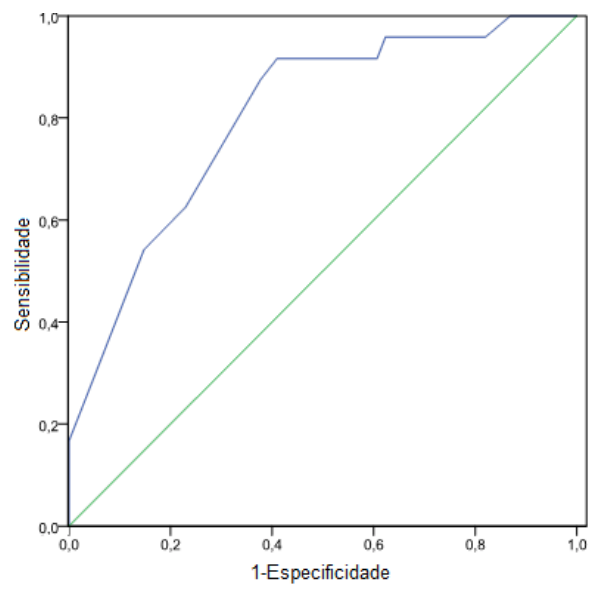


Figura 3. Curvas ROC do TOP-J/9-Br

(a) Controle versus CCL

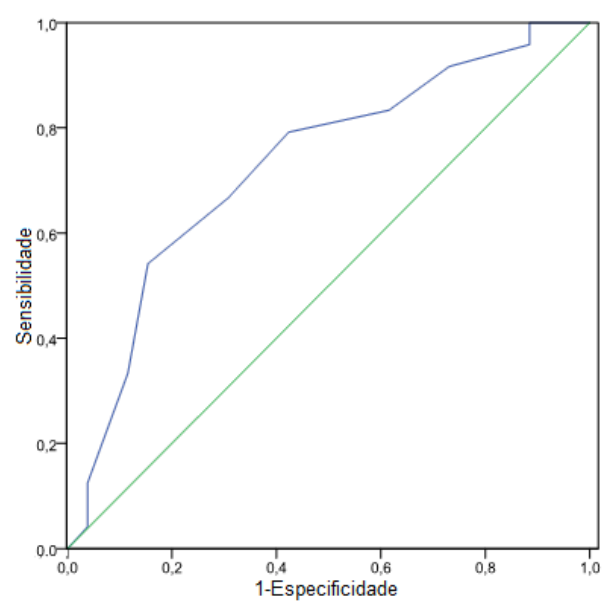

(c) Controle versus DFT

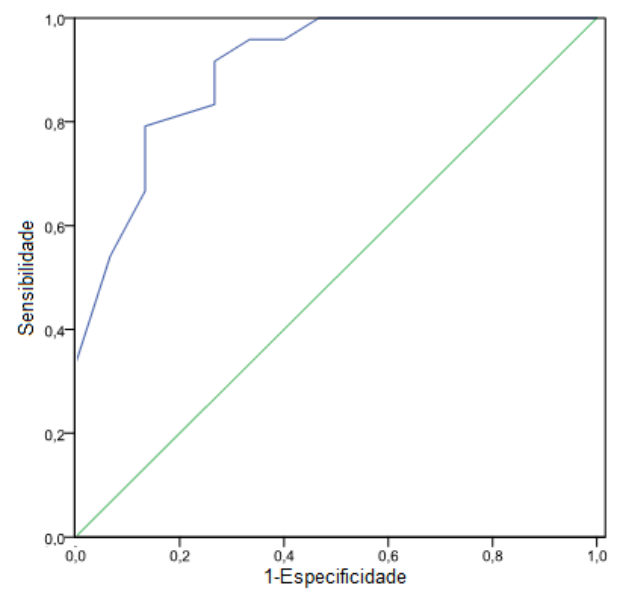

(b) Controle versus DA

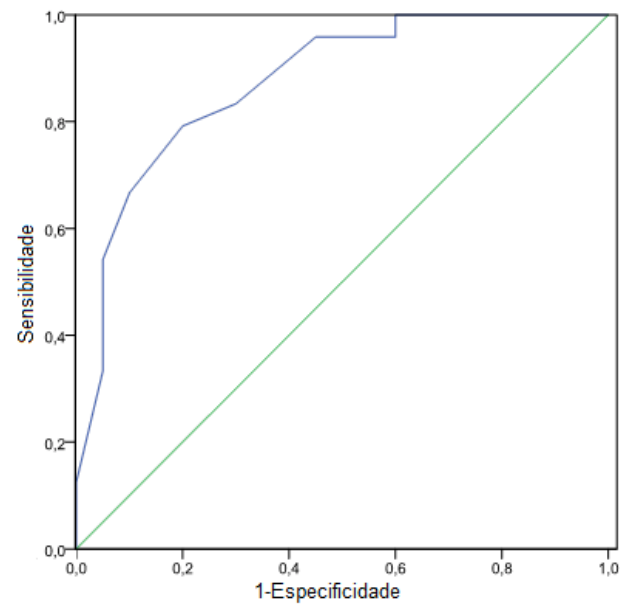

(d) Controle versus Demência

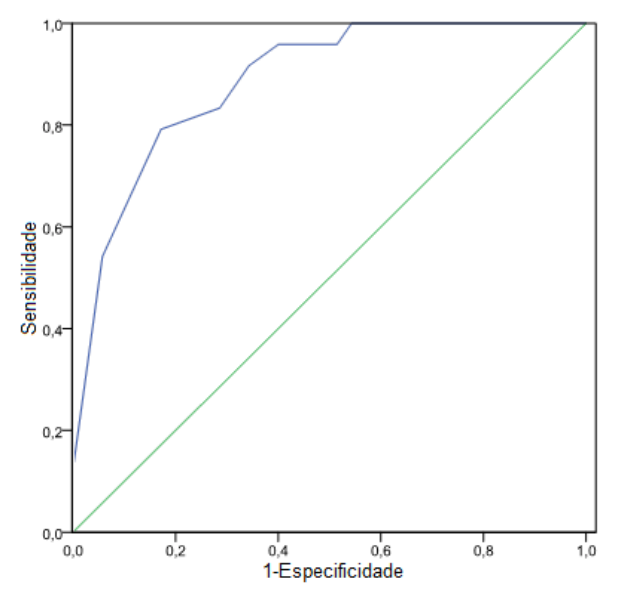

(e) Controle versus Paciente

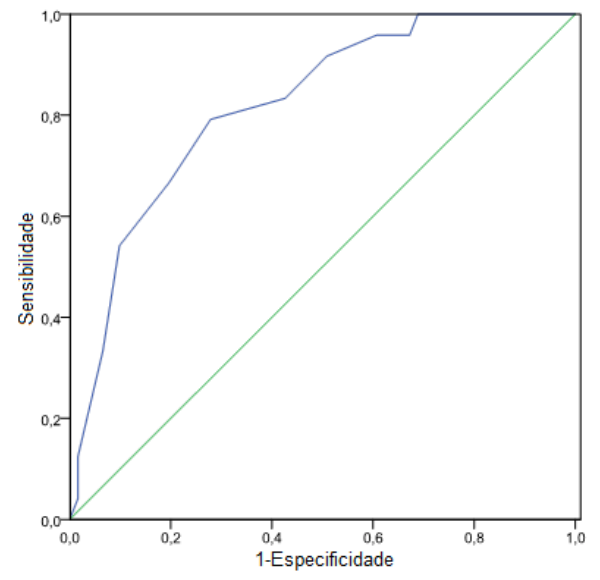




\section{DISCUSSÃO}

\subsection{TOP-J}

O TOP-J é um teste breve e objetivo de julgamento prático cotidiano desenvolvido para uso em adultos mais velhos. Os autores objetivaram o desenvolvimento de uma escala com questões representativas dos problemas de julgamento frequentemente vivenciadas por estes indivíduos, que abrangessem quatro domínios: segurança, médico, financeiro e social/ético. Os itens foram elaborados com base em revisão da literatura sobre julgamento e constructos relacionados, pesquisa com neuropsicólogos, e informações de participantes de um estudo longitudinal e seus acompanhantes. Os itens são de fácil compreensão, mas complexos o suficiente para exigir resolução ativa de problemas e consideração sobre opções e potenciais consequências. Foram criados para minimizar a influência de aspectos emocionais e sociais, bem como conhecimento cristalizado.

O TOP-J original foi gentilmente cedido pela autora, para a realização deste estudo. O TOP-J/15 foi traduzido e algumas questões foram adaptadas para uso em amostra da população brasileira. A partir da versão brasileira de 15 itens (TOP-J/15Br), obtivemos a escala brasileira reduzida de 9 itens (TOP-J/9-Br).

\subsection{Amostra estudada}

Os grupos foram homogêneos quanto ao gênero e escolaridade, e heterogêneos quanto à idade. Indivíduos do grupo CCL e do grupo DA foram mais velhos que indivíduos do grupo controle e do grupo DFT.

Não é surpreendente que os pacientes com DFT tenham sido mais jovens que os indivíduos dos outros subgrupos clínicos, visto que na DFT as manifestações clínicas são 
mais precoces do que as de outras etiologias de comprometimento cognitivo (Piguet et al., 2011, Josephs et al., 2011). Apesar de mais jovens, os pacientes do grupo DFT não tiveram melhor desempenho do que pacientes do grupo DA no TOP-J. Este achado está de acordo com a hipótese de que os efeitos da idade dependem do contexto, da complexidade da tarefa (Brand \& Markowitsch 2009) e relevância ecológica. O TOP-J parece ativar funções mais resistentes aos efeitos da idade, como conhecimento (procedural e declarativo) e memórias episódicas relevantes, habilidades que facilitam a geração de soluções, e podem compensar o declínio cognitivo pela experiência e prática (Liu et al., 2007). Não existem estudos comparando o desempenho nos testes de julgamento em diferentes idades.

\subsection{Evidências relacionadas à Validade Baseada na Estrutura Interna e}

\section{Redução da Escala}

O TOP-J/15 e o TOP-J/9 demonstraram propriedades psicométricas adequadas. Os resultados do teste de Esfericidade de Bartlett, que permite identificar se as variáveis se encontram relacionadas, e do teste de medida da adequação da amostragem KaiserMeyer-Olkin $(\mathrm{KMO}=0,64)$, que reflete a homogeneidade das variáveis indicaram, respectivamente, que houve correlação entre os itens avaliados do TOP-J e que a amostra deste estudo estava adequada para a realização da análise fatorial exploratória.

Assim, realizou-se a análise fatorial no TOP-J/15-Br, que indicou os cinco fatores com valores próprios superiores a 1 (mais especificamente 1,07 a 2,98), que juntos, respondiam por $54 \%$ da variância total. Este valor é similar ao encontrado no estudo de Rabin et al. $\left(2013^{1}\right)$, onde foram também identificados seis fatores, com valores próprios superiores a 1 , representando $55,9 \%$ da variância total. 
A possibilidade de redução de itens foi verificada a partir da análise fatorial, cujo objetivo é identificar fatores que resumam as variáveis, para então tomar a decisão sobre quais cargas fatoriais devem ser consideradas. Em síntese, considera-se que as cargas fatoriais maiores que $\pm 0,30$ atingem o nível mínimo; cargas de $\pm 0,40$ são consideradas mais importantes; e se as cargas são de $\pm 0,50$ ou maiores, elas são consideradas com significância prática, embora cargas extremamente altas $(0,80$ ou superiores $)$ não são comuns. Portanto, a significância prática das cargas é um critério importante a ser considerado. Considerando que a metodologia estatística não substitui o conhecimento específico que o pesquisador tem que ter em seu próprio campo de atuação (Benze, 2001), a redução da escala brasileira foi realizada conforme o trabalho original (Rabin et al., 2007), no qual, inicialmente, optou-se pela extração de um único fator, tendo-se em vista a identificação de itens que obtivessem carga fatorial inferior a 0,4 e portanto, não se mostrassem tão relevantes para a análise do construto julgamento, podendo ser excluídos.

Assim como no estudo original, neste estudo também foram identificados seis itens com cargas fatoriais abaixo de 0,4 , os quais foram retirados. Os itens mantidos no TOP-J/9-Br abrangeram todos os domínios propostos pelos autores, dentre eles dois de domínio médico (itens 1 e 4); dois de domínio financeiro (itens 2 e 3), quatro de domínio social/ético (itens 6, 7, 8 e 9), e um de domínio segurança (item 5). Os itens mantidos no TOP-J/9 original (Rabin et al., 2007) não foram exatamente os mesmos mantidos no TOP-J/9-Br e tais versões tiveram 7 itens em comum (itens 1, 2, 4, 6, 7, 8 e 12 do TOP$\mathrm{J} / 15)$.

Não é surpreendente que tenha havido diferenças entre o TOP-J/9-Br e o TOP-J/9 original, visto que além de pertencentes a culturas diferentes, as amostras não foram equiparadas. No estudo original os indivíduos aparentemente tinham idade maior e eram 
mais escolarizados, o que era previsto já que um dos objetivos deste estudo foi a adaptação do TOP-J para uma cultura diferente e menos escolarizada, para verificar se a acurácia para distinguir controles de pacientes iria se manter nesta população. Além disso, foram feitas adaptações em alguns dos itens de acordo com nossa realidade.

Outra análise fatorial exploratória para um único fator foi realizada com os 9 itens restantes. As cargas fatoriais de cada um dos itens sobre o fator geral foram maiores que 0,4 , assim como observado no estudo original. Isto aponta para o fato de que, apensar dos itens mantidos nesta versão terem sido diferentes do TOP-J/9 original para duas questões, os itens mantidos parecem ser adequados para a população brasileira.

\subsection{Evidências relacionadas à Validade de Critério}

As validades de critério do TOP-J/15- $\mathrm{Br}$ e do TOP-J/9-Br também foram verificadas e mostraram-se adequadas. No teste Kruskal-Wallis observou-se que os escores totais do TOP-J/15-Br e do TOP-J/9-Br, foram significativamente diferentes para alguns pares de grupos. Pelo teste Bonferroni, observou-se que, nos escores totais do TOP-J/15-Br e do TOP-J/9-Br, houve diferença estatisticamente significativa de desempenho na comparação entre controles e CCL, controle e DA, controle e DFT, CCL e DA, e CCL e DFT. Não houve diferença estatisticamente significativa no desempenho de DA e DFT nos escores totais do TOP-J/15-Br e do TOP-J/9-Br.

A cognição social refere-se aos processos psicológicos que capacitam o indivíduo a interagir com outras pessoas e a participar de um grupo social (Frith, 2008). Um modelo conceitual de cognição social a caracteriza como englobando as habilidades de: 1) percepção social, 2) teoria da mente, 3) percepção emocional e 4) estilo de atribuição (tendência individual de explicar causas e eventos da própria vida). A percepção social consiste na habilidade de extrair pistas (sociais e emocionais) de um 
contexto social, e é relacionada ao conhecimento de normas sociais (Couture et al., 2006).

A tomada de decisão está relacionada aos processos de avaliação da relevância de sinais sociais e emocionais, e com a geração de respostas comportamentais (Evans, 2008; Shany-U e Rankin, 2011). O processo avaliativo da tomada de decisão é denominado julgamento. Portanto, julgamento além de aspectos cognitivos (funções executivas, memoria, linguagem, atenção e raciocínio), está relacionado, embora em menor escala, com aspectos emocionais e sociais (Evans, 2008; Rabin et al., 2007).

Embora pacientes com demência possam executar algumas tarefas rotineiras de forma relativamente adequada, habilidades de resolução de problemas relacionados ao trabalho e ambientes sociais e domésticos são frequentemente afetados. Em situações não estruturadas, prejuízo em habilidades executivas potencialmente levam a um pior julgamento em situações diárias, tais como decisões impulsivas, exploração inadequada de questões pertinentes, fixação em uma única solução sem habilidade de alternar entre uma e outra, ou julgamento baseado nas consequências imediatas e não a longo prazo (Channon, 2004). Assim, a perda de capacidade de julgamento é comum em demência e pode levar a comportamentos sociais inadequados e de risco (Rabin et al., 2007).

Como demência está associada com uma perda de funcionamento independente, medidas de avaliação de julgamento podem ser úteis para identificar, apoiar ou rejeitar a possibilidade de o paciente estar apto a engajar-se em tarefas complexas que exijam julgamento prático em tarefas cotidianas. Julgamento deve ser parte da avaliação de idosos com suspeita de demência e medidas de sua avaliação podem ser úteis para informar sobre o diagnóstico, dificuldades (e ajudar a evitar consequências perigosas), 
auxiliar no tratamento, como por exemplo, no desenvolvimento de programas de reabilitação, e em aspectos legais, como interdições.

\section{CCL}

É interessante notar que pacientes com CCL tiveram pior desempenho, e consequentemente demonstraram pior capacidade de julgamento do que controles, no TOP-J. Estudos têm demonstrado que pacientes com CCL muitas vezes apresentam declínio em funções executivas e deficiência em certas atividades funcionais da vida diária que envolvem raciocínio complexo (Jefferson et al., 2008; Perneczky 2006). Há evidências crescentes sugerindo que dificuldades de resolução de problemas mensurados com escalas de AIVD ou medidas neuropsicológicas tradicionais (Farias et al., 2006; Kounti et al., 2006; Perneczky et al., 2006; Wadley et al, 2007), tomada de decisão mensurada por testes de aposta (Zamarian et al., 2010), e tarefas de resolução de problemas (Burton et al., 2009a; Burton et al., 2009b) podem ser observadas em adultos mais velhos com CCL.

A constatação de que julgamento é prejudicado em CCL, se comparados a controles é consistente com os resultados empíricos observados em outros estudos, como os estudos de validação do TOP-J/15 original (Rabin et al., 2005² - média controles $=37,6(3,9)$, média $\mathrm{CCL}=34.1(4.2)$ e média $\mathrm{DA}=28,4(6,1)$ e do TOP-J/9 (Rabin et al., 2007 - média controles $=23,0 \mathrm{DP}=2,4$; média $\mathrm{CCL}=20,2 \mathrm{DP}=3,4$; média $\mathrm{DA}=16,2 \mathrm{DP}=4,8)$, nos quais os controles tiveram desempenho significativamente inferior a CCL e DA, e CCL tiveram desempenho significativamente inferior a DA. Em estudo realizado recentemente com o TOP-J/15 original (Rabin et al., 2013 ${ }^{1}$ ) (média controles $35,0(5,0)$ média $\mathrm{CCL}=31,7(5,0)$, também foi observado melhor desempenho em controles do que em CCL. 
Entretanto, Rabin et al. (2007) verificaram que o NCSE não foi capaz de distinguir CCL de controles. Isto reforça a hipótese de que declínio na capacidade de julgamento, assim como no funcionamento diário destes pacientes, depende da sensibilidade do método de avaliação usado para detectar as alterações sofridas (Farias et al., 2006). Uma avaliação contendo um escopo mais amplo de funções executivas, que engloba julgamento prático e raciocínio complexo poderia fazer uma ferramenta de rastreio cognitivo mais forte (Mansbach et al., 2013). O TOP-J/9 tem se mostrado um bom instrumento para esta finalidade. Não há estudos verificando o desempenho de CCL comparado a controles com outras medidas de julgamento disponíveis.

\section{DA versus DFT}

Foi interessante, mas não surpreendente, que não tenha havido diferença estatisticamente significativa entre pacientes com DA e pacientes com DFT. O TOP-J tem por objetivo ser ecologicamente representativo dos problemas rotineiramente vivenciados por adultos mais velhos, envolvendo menos processos emocionais e mais processos cognitivos de alta ordem. Em um estudo de investigação da relação entre neuroimagem estrutural e julgamento prático em adultos mais velhos, Rabin et al. (2009) verificaram que baixo desempenho no TOP-J foi correlacionado com atrofia no giro frontal inferior esquerdo e, em menor extensão, com o giro frontal superior.

Resolução de problemas diários é um conceito multifacetado que envolve diversas habilidades, incluindo processos cognitivos, sociais, e emocionais, e conhecimento prático derivado de experiências diretas e aprendizado social ao longo da vida. Os processos cognitivos parecem associados ao córtex pré-frontal lateral incluindo manipulação de conhecimento prévio relevante para apreciar a situação problema, geração de estratégias apropriadas para abordá-lo, inibição de respostas inapropriadas, monitoramento e julgamento da eficácia das soluções dos problemas, atentar para as 
informações pertinentes identificando objetivos apropriados, considerando conhecimentos sociais e práticos relevantes, atentando para consequências potenciais futuras dos diferentes cursos de ação de diferentes perspectivas, fazer julgamentos racionais comparativos sobre eles, avaliando o sucesso de possíveis consequências.

Do ponto de vista neuropsicológico, envolve principalmente atenção, funções executivas (gerar e utilizar estratégias apropriadas, manter a informação na memória operacional, alternar entre uma ideia e outra, inibir respostas inapropriadas), memória (lembrar do desfecho em situações semelhantes anteriormente vivenciadas) e linguagem (ter boa capacidade de compreensão e de expressão das respostas escolhidas) (Channon, 2004, Rabin et al., 2007).

É importante não confundir julgamento prático, mensurado pelo TOP-J, com julgamento moral ou social, definido como julgamento avaliativo da adequação do comportamento no contexto das percepções sociais de certo e errado (Moll et a., 2005) e relacionado com córtex ventromedial e amígdala (Greene et al., 2001, Mendez et al., 2005; Ciaramelli et al., 2007), e que é tipicamente mais afetado em pacientes com DFT do que em pacientes com DA.

Na DFT há grande proeminência de disfunções de circuitos frontais orbito mediais nas fases iniciais, e posterior acometimento de circuitos dorsolaterais. Por este motivo, testes cognitivos clássicos usualmente utilizados para avaliar quadros demenciais, sensíveis a funções dorsolaterais como parece ser o caso do TOP-J, podem falhar no diagnóstico de DFT em fase inicial (Torralva et al., 2009), ao passo que testes que avaliam funções orbito mediais da cognição social, incluindo reconhecimento de emoções básicas, tomada de decisão social, inferência sobre estados mentais de outras pessoas e consciência do seu próprio comportamento social e julgamento moral têm se 
mostrado sensíveis na detecção das fases iniciais da doença(Moll et a., 2005; Cerami e Cappa, 2013; Torralva et al., 2009).

Como exemplo, no estudo de Mendez et al., (2005), o julgamento moral foi avaliado em um primeiro momento, com o questionário Moral Behavior Inventory (Rettig et al., 1959 apud Mendez et al., 2005) no qual o indivíduo é solicitado a marcar 1não errado, 2 - levemente errado, 3 - moderadamente errado, 4 - muito errado, a itens do tipo falhar em manter promessas, dirigir depois de beber, pegar o maior pedaço de uma torta, pedir para outros fazerem parte de sua tarefa de casa, dentre outros. Em um segundo momento, os pacientes foram avaliados com dois dilemas de julgamento propostos por Greene et al. (2001), por exemplo: "Imagine que um pequeno trem desgovernado sobre trilhos está se aproximando de cinco operários que irão morrer se ele continuar. Você está em uma passarela sobre os trilhos, entre o trem e cinco trabalhadores. Próximo a você, na passarela há um estranho. A única maneira de salvar a vida de cinco operários é empurrar esse estranho fora da ponte para o seu corpo fazer o bonde parar. O estranho vai morrer se você fizer isso, mas os cinco trabalhadores serão salvos." Você empurraria o estranho para salvar os cinco trabalhadores?“‘. Pacientes com DA tiveram melhor desempenho nesta tarefa do que pacientes com DFT, que geralmente têm distúrbios de comportamento moral ou habilidade de serem éticos e aceitarem normas e regras (Mendez et al., 2005).

Muitos estudos têm se dedicado a investigar habilidades de tomada de decisão em indivíduos com DFT (Torralva et al., 2007, Torralva et al., 2009, Gleitcherrich et al., 2010) e sua comparação com comprometimento cognitivo de outra etiologia, mas somente um estudo além deste verificou o comprometimento de julgamento prático em pacientes com DFT. 
Baldock et al. (2014) encontraram diferença estatisticamente significativa $(p=$ 0,027) nos escores do TOP-J em grupos de 9 pacientes com DA e 9 pacientes com DFT, pareados com base nos escores do teste MOCA. Pacientes com DFT tiveram escores com media de 15,89 e pacientes com DA tiveram media de 20,89 (média para escore "normal" é 19,4). Pelos escores, parece que foi usado o TOP-J/9, embora os autores não tenham especificado. Além disso, eles não mencionaram se os grupos foram pareados por escolaridade e nem a intensidade das demências. Talvez diferença entre os dois grupos tenha sido observada neste trabalho e não no nosso pelo fato de os pacientes terem demência moderada ou grave, quando as diferenças clínicas tornam-se mais óbvias; porque os grupos não foram pareados por educação; porque a amostra é menor ou simplesmente por causa das diferenças demográficas e socioculturais entre as amostras.

\subsection{Evidências relacionadas à Validade de Constructo}

A validade de construto (validade convergente e discriminante) foi avaliada por correlações entre o TOP-J/15-Br e TOP-J/9-Br e os testes Compreensão e GDS.

Evidências de validade convergente foram encontradas no TOP-J/15-Br (rho= $0,49, \mathrm{p}=0,01)$ e TOP-J/9 (rho $=0,51, \mathrm{p}=0,01)$ por correlações significativas positivas com o subteste WAIS Compreensão, uma medida comumente utilizada na avaliação de julgamento (Rabin et al., 2008), que avalia habilidades como abstração verbal, entendimento de normas sociais, julgamento moral e planejamento, necessários para a realização de um bom julgamento prático. A correlação do TOP-J/15-Br com o MZ, medida de planejamento e habilidade de seguir regras, apoia a validade convergente.

A ausência de correlação entre o TOP-J/15-Br (rho=0,21; $\mathrm{p}=0,32)$ e TOP-J/9-Br ( $r$ ho $=0,25 ; \mathrm{p}=0,24)$ e medida humor (GDS) apoia a evidência de validade discriminante, também observada por Rabin et al., 2007, apontando para a hipótese de que o TOP-J 
envolve mais aspectos cognitivos do que emocionais, avaliando julgamento prático, e não julgamento moral.

\subsection{Confiabilidade}

A confiabilidade foi estabelecida através da consistência interna, determinada pelo alfa de Cronbach. Com este método, a consistência da escala é determinada pelas inter-relações entre os itens, considerando o número total de itens que a escala compreende e estimando a proporção da variância que é consistente em um conjunto de escores de teste. A consistência interna de confiabilidade das pontuações do TOP-J/15 neste estudo, estimado pelo alfa de Cronbach foi 0,69. Este valor está situado dentro do intervalo aceitável para notas de corte de testes em ciências sociais, que variam de 0,6 a 0,8 (Nunnally e Bernstein, 1994 apud Rabin et al., 2007) e equipara-se aos valores de 0,68 e 0,6 verificados para o TOP-J/15 nos estudos de Rabin et al., 2005 e Rabin et al., 2013 respectivamente.

No TOP-J/9 deste estudo, o coeficiente $\alpha$ de Cronbach foi de 0,68 ( $\mathrm{p}<0,001)$, que equipara-se ao valor de 0,63 obtido no TOP-J/9 original (Rabin et al., 2007). Os valores alfa de Cronbach do TOP-J/15 e do TOP-J/9 deste estudo e dos estudos originais comparam-se favoravelmente com o alfa do NAB JDC de 0,45 (Stern e White, 2003), com os valores de alfa do NCSE JQ de 0,04 e 0,46 encontrados respectivamente para controles e pacientes com DA (Woods et al., 2000) e com o valor de 0,07 ( $\mathrm{p}>0,05)$ encontrado no NCSE JQ por Rabin e colaboradores (2007). MacDougall e Mansbach (2013) encontraram alfa de Cronbach de 0,83 para o NAB JDC. Apesar de ter sido um valor alto, os dados foram coletados em amostra de residentes em instituição de longa permanência, o que pode limitar a generalização dos achados. Além disso, os autores não incluíram sujeitos controles no estudo. Mansbach et al. (2013) encontraram para o KPT 
um alfa de 0,88 e 0,93 em estudos realizados com amostras de 121 e 163 participantes, respectivamente. Novamente os dados podem não ser generalizáveis por terem sido coletados em indivíduos residentes em instituição de longa permanência. Além disso, segundo os autores, a pontuação do teste parece ter elemento de subjetividade e pode ter havido variabilidade na pontuação atribuída pelos profissionais da saúde com diferentes formações, que participaram da coleta.

O valor observado de alfa de Cronbach neste estudo para o TOP-J/15 foi equiparado ao valor encontrado para o TOP-J/9. Ambos são valores de alfa dentro do valor aceitável para ciências sociais, e não tão altos, o que é esperado pela própria constituição do teste, que se propõe a avaliar o constructo julgamento através de diferentes domínios relacionados.

Ambas as versões apresentaram boa consistência interna devido ao fato de o coeficiente geral estar próximo de 0,7. Observou-se também que os itens medem o mesmo constructo e que a análise de consistência interna não sugeriu a exclusão de qualquer outro item para aumentar a confiabilidade do questionário, pois os valores de alfa para cada item estiveram próximos do alfa geral, variando de 0,63 a 0,69 no TOP$\mathrm{J} / 15-\mathrm{Br}$, em que o Alfa de Cronbach foi 0,69 , e de 0,58 a 0,67 no TOP-J/9-Br, em que o Alfa de Cronbach foi 0,68 .

\subsection{Sensibilidade e Especificidade}

A acurácia de um teste é considerada com relação a alguma forma de se saber se a doença está realmente presente ou não - uma indicação mais sólida da verdade, frequentemente referida como padrão-ouro. A sensibilidade é definida como a proporção de pessoas com a doença, que tem um teste positivo. Os testes sensíveis raramente são negativos na presença da doença, eles produzem poucos falso-negativos. 
Especificidade é a proporção de indivíduos sem a doença que têm um teste negativo. Os testes específicos raramente são positivos na ausência da doença, eles produzem poucos resultados falso-positivos (Fletcher e Fletcher, 2006).

A curva ROC é uma forma de expressar a relação entre a sensibilidade e a especificidade de um teste. Ela é construída pela representação gráfica da taxa de verdadeiro positivo (sensibilidade) contra uma taxa de falso positivo (1-especificidade) ao longo de uma faixa de possíveis pontos de corte. Os valores nos eixos vão de probabilidade de 0 a 1 . Especificidade é então verdadeiro negativo, e falso negativo é 1 - sensibilidade. Obviamente, é necessário que um teste seja ao mesmo tempo sensível e específico, mas isto nem sempre é possível. O contrabalanço entre a sensibilidade e a especificidade é importante sempre que os valores clínicos assumem uma gama de valores. Nessas situações, a localização de um ponto de corte, decisão entre o normal e o anormal, é uma decisão arbitrária. Geralmente o melhor ponto de corte fica no "ombro" da curva ROC. Os testes com bom poder discriminatório concentram-se na parte superior esquerda da curva. A acurácia geral de um teste pode ser descrita como área sobre a curva e quanto maior for a área, melhor será o teste (Fletcher e Fletcher, 2006).

Neste estudo, optamos por priorizar o maior valor de sensibilidade, com a maior especificidade possível, que não reduza o valor da sensibilidade para diminuir a chance de erros do tipo falso-negativo. Um teste sensível (isto é, um teste que seja geralmente positivo na presença da doença) precisa ser escolhido quando as consequências de se deixar passar uma doença são consideráveis, como no caso de pacientes com demência com déficits de julgamento, que se não identificados podem persistir em comportamentos não seguros como utilizar fogão, dirigir, administrar 
finanças ou utilizar medicações prescritas sem assistência. Pacientes e seus familiares precisam ser instruídos sobre as consequências de déficits na habilidade de julgamento e a relação dos sintomas com o processo da doença (Rabin et al., 2007). Os testes sensíveis também são úteis nos estágios iniciais de um processo diagnóstico, quando diversas possibilidades estão sendo consideradas, para reduzir o número das mesmas (Fletcher e Fletcher, 2006).

No caso do TOP-J/15-Br e do TOP-J/9-Br, os pontos de corte com alta sensibilidade sugerem que, pacientes com dificuldade de julgamento terão grande probabilidade de ser identificados. Se o resultado for igual ou acima do ponto de corte é porque há muitas chances de o sujeito ter suas habilidades de julgamento preservadas. Entretanto, pelo fato de a especificidade ter sido menor, alguns pacientes identificados com dificuldade de julgamento poderão não ter. Por isso, nós recomendamos uma anamnese com história detalhada e uma avaliação neuropsicológica abrangente em casos em que, embora o TOP-J tenha apontado para déficit de julgamento, o clínico achar que o paciente tem suas capacidades de julgamento prático diário preservadas.

Vale ressaltar que o TOP-J tem por objetivo avaliar a capacidade de julgamento prático e não se trata de um teste diagnóstico ou de rastreamento de demência. Avaliação da acurácia é importante para comprovar a consistência do teste para o propósito a que se destina.

O ponto de corte para diferenciar controles de pacientes no TOP-J/15 foi 30 $($ máximo=45) e no TOP-J/9 foi 19 (máximo=27).

Os resultados indicaram que tanto o TOP-J/15 quanto o TOP-J/9 apresentaram boa acurácia para discriminar controles de pacientes com CCL (pontos de corte 31 e 19, respectivamente); controles de pacientes com DA (pontos de corte 29 e 18); controles de pacientes com DFT (pontos de corte 30 e 18); controles de pacientes com 
demência (DA + DFT) (pontos de corte 30 e 18) e grupo controle do grupo de pacientes $(\mathrm{CCL}+$ demência) (pontos de corte 30 e 19).

Ainda não foram realizados estudos que verificassem a sensibilidade e especificidade do TOP-J original de 9 e de 15 itens. Outros estudos além deste verificaram a acurácia de outros testes de julgamento. Em estudo realizado com o NAB JDC, este teste demonstrou sensibilidade de 0,61 e especificidade de 0,88, para distinguir pacientes com DA de pacientes com diagnósticos não especificados, sem grupo controle (Gavett et al., 2012). Em outro estudo realizado com o KPT, o ponto de corte encontrado representou uma sensibilidade de 0,85 e especificidade de 0,72 , mas os autores não especificam os tipos de demência e demais subgrupos clínicos comparados (Mansbach et al., 2013).

Com base nos resultados, se quisermos priorizar um diagnóstico precoce de distúrbio de julgamento, necessário por exemplo para fins legais, devemos optar pelos escores de maior sensibilidade, com um valor razoável de especificidade. Do ponto de vista clínico, recomendamos uma pontuação de corte de 30 para o TOP-J/15 e de 19 para o TOP-J/9, para diferenciar pessoas com capacidade de julgamento comprometida daquelas com capacidade de julgamento preservada. Estas pontuações produziram maior sensibilidade do que especificidade no TOP-J/15 e no TOP-J/9 na discriminação de julgamento de todos os grupos avaliados.

Os resultados sugerem que quando quisermos priorizar a identificação de um distúrbio precoce de julgamento na população brasileira, com avaliação breve, o TOP-J/9 deve ser usado como medida de rastreio.

Em avaliação neuropsicológica mais abrangente, o TOP-J/15 é também recomendado, visto que, apesar de na comparação entre controles e CCL ele ter tido especificidade (46,2\%) mais baixa, ele demonstrou alta sensibilidade (87,5\%). Além 
disto, na identificação de pacientes com diferentes graus de comprometimento cognitivo, ele apresentou alta sensibilidade (91,7\%), com maior especificidade (59\%) e pode fornecer uma análise qualitativa dos itens comprometidos, necessários por exemplo para programas de reabilitação, comprovadamente úteis para pacientes com alterações cerebrais menos graves (Yassuda e Nunes, 2009).

\subsection{Considerações finais}

Em síntese, os itens do TOP-J foram desenvolvidos para serem particularmente sensíveis a prejuízo de julgamento prático em adultos e idosos. Nossos resultados sugerem que o TOP-J demonstrou propriedades psicométricas robustas para o uso pretendido com a população brasileira.

A validade de estrutura interna foi verificada e esteve adequada. A análise fatorial exploratória rendeu cinco fatores com autovalores maiores que 1 que responderam por $55 \%$ da variância total. Posteriormente, foi realizada a extração para um único fator, e 6 itens com carga inferior a 0,4 foram retirados do TOP-J/15-Br. Em seguida, uma nova análise fatorial com extração de um fator foi realizada com o TOP-J/9-Br. A validade de critério também esteve adequada, visto que os escores foram estatisticamente diferentes entre grupos controle, CCL, DA e DFT; CCL e DA; e CCL e DFT. Os escores não foram significativamente diferentes entre DA e DFT, o que pode ser esperado pelo fato de que o TOP-J avalia julgamento prático e não julgamento moral, exige menos processamento emocional e mais funções cognitivas complexas, relacionadas ao córtex orbito frontal dorsolateral e menos sensíveis para distinção entre DFT precoce e DA. O TOP-J/15 e TOP-J/9 apresentaram validade de constructo adequadas, observada, no TOP-J/15 por correlações significativas com o MZ e com o Compreensão, e no TOP-J/9 com o teste 
Compreensão, medidas neuropsicológicas que avaliam funções executivas (particularmente planejamento), compreensão de problemas cotidianos e entendimento de regras e conceitos sociais. A ausência de correlação significativa com a GDS apontou para validade divergente.

A confiabilidade de ambos TOP-J/15 e TOP-J/9 apresentaram valores aceitáveis e equiparados favoravelmente em relação aos estudos com o TOP-J original.

Finalmente, a acurácia do TOP-J para a detecção de comprometimento de julgamento foi muito boa, com uma sensibilidade de $91,7 \%$ e especificidade de $59 \%$ no TOP-J/15 e sensibilidade de $79,2 \%$ e especificidade de $72,1 \%$ no TOP-J/9, e uma área sob a curva ROC respectivamente de 0,80 e de 0,82 .

Para investigação inicial e isolada da capacidade de julgamento, recomenda-se preferencialmente o uso do TOP-J/9, por ser breve e ter apresentado boa sensibilidade e especificidade, já na identificação de CCL. Para uso em avaliação abrangente, o TOP$\mathrm{J} / 15$ pode ser utilizado visto que possibilita avaliação qualitativa mais abrangente (o que pode ser útil para programas de reabilitação) e, apesar de ter demonstrado especificidade razoável na identificação de CCL, ele apresentou melhor sensibilidade distinção de indivíduos cognitivamente saudáveis de pacientes com graus variados de comprometimento cognitivo, com ótima sensibilidade. 


\section{CONCLUSÕES}

O TOP-J é o primeiro teste de julgamento a ser avaliado em nosso meio, em população pouco escolarizada e este é o primeiro estudo de investigação da acurácia desempenho de pacientes com DFT em teste específico de julgamento e a comparar o desempenho destes pacientes com o desempenho de CCL. Os resultados estiveram dentro do esperado, e confirmaram a hipótese de prejuízo de julgamento já em CCL, visto que o TOP-J diferenciou julgamento em espectro de comprometimento cognitivo, distinguindo controle de CCL e CCL de dementes. As versões brasileiras do TOP-J demonstraram propriedades psicométricas adequadas e provaram ser medidas adequadas para o propósito a que se destinam.

Recomendamos que o TOP-J seja usado como instrumento de avaliação da habilidade de julgamento prático cotidiano em indivíduos mais velhos no contexto clínico, podendo ter seu uso estendido para investigação desta capacidade para fins legais. Não recomendamos o uso do TOP-J em indivíduos com distúrbios de linguagem. 


\section{ANEXO A - APROVAÇÃO DO COMITÊ DE ÉTICA}

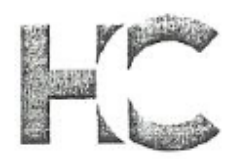

\section{APROVAÇĀO}

A Comissão de Ética para Análise de Projetos de Pesquisa CAPPesq da Diretoria Clínica do Hospital das Clínicas da Faculdade de Medicina da Universidade de Sāo Paulo, em sessão de 08/06/2011. APROVOU o Protocolo de Pesquisa $n^{\circ}$ 0076/11, intitulado: 'TEST OF PRACTICAL JUDGMENT (TOP-J): ADAPTAÇÃO BRASILEIRA EM AMOSTRA DE INDIVÍDUOS COGNITIVAMENTE SAUDÁVEIS, COM COMPROMETIMENTO COGNITIVO LEVE E DEMÊNCIA", apresentado pelo Departamento de NEUROLOGIA, inclusive o Termo de Consentimento Livre e Esclarecido.

Cabe ao pesquisador elaborar e apresentar à CAPPesq, os relatórios parciais e final sobre a pesquisa (Resolução do Conselho Nacional de Saúde n 196, de 10/10/1996, inciso IX.2, letra "c").

Pesquisador (a) Responsável: Dra. Soni Maria Dozzi Brucki

Pesquisador (a) Executante: Patrícia Helena Figueirêdo do Vale Capucho Co-autores:

CAPPesq, 08 de Junho de 2011

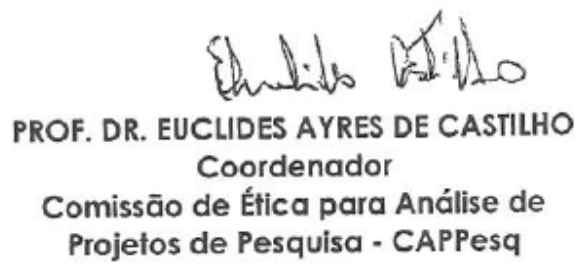

Comissão de Ética para Análise de Projetos de Pesquisa do HCFMUSP da Diretoria Clinica do Hospital das Clínicas da Faculdade de Medicina da Universidade de Săo Paulo Rua Ovidio Pires de Campos, 225, $5^{\circ}$ andar - CEP 05403010 - Săo Paulo - SP Fone: 01130696442 Fax: 01130696492 e-mail: cappesq@hcnet.usp.br 


\title{
ANEXO B - TERMO DE CONSENTIMENTO LIVRE E ESCLARECIDO \\ hospital das Clínicas da faCuldade de medicina da UNIVERSIDAdE dE SÃO PAULO-HCFMUSP
}

\author{
TERMO DE CONSENTIMENTO LIVRE E ESCLARECIDO
}

DADOS DE IDENTIFICAÇÃO DO SUJEITO DA PESQUISA OU RESPONSÁVEL LEGAL

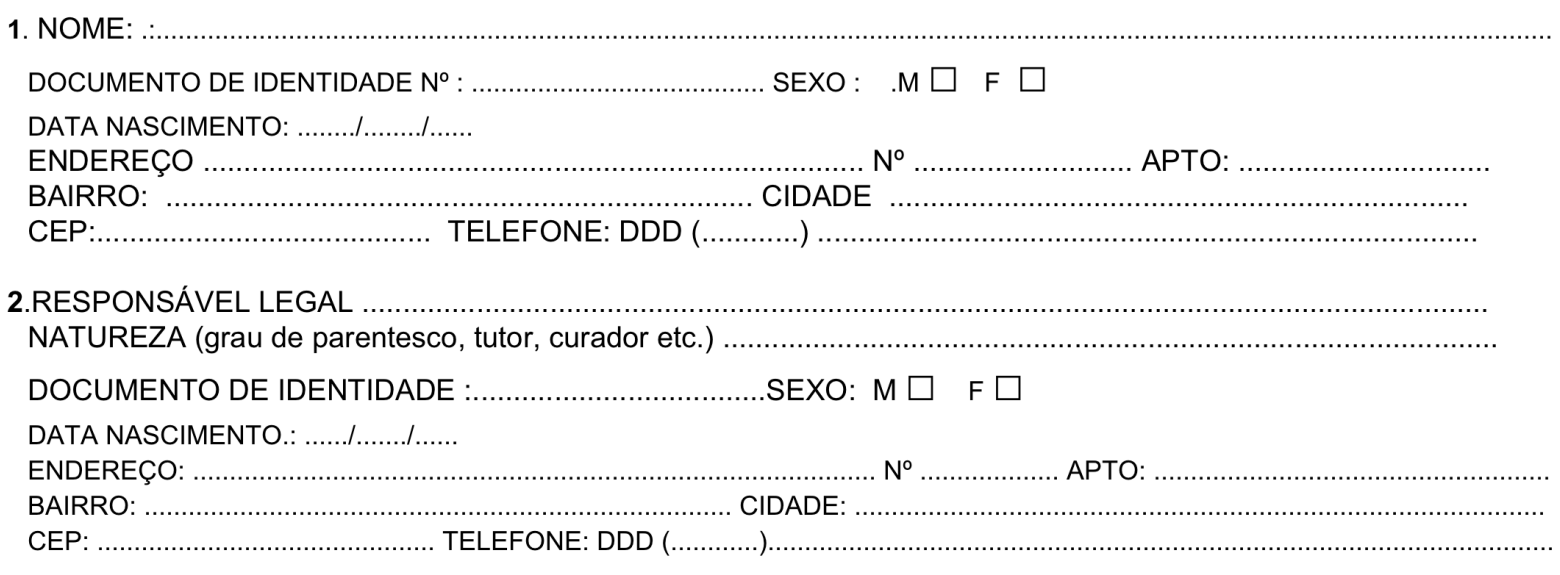

\section{DADOS SOBRE A PESQUISA}

1. TÍTULO DO PROTOCOLO DE PESQUISA: Test of Practical Judgment (TOP-J): Adaptação brasileira em amostra indivíduos cognitivamente saudáveis, com Comprometimento Cognitivo Leve e Demência

PESQUISADOR RESPONSÁVEL: Dra. Sonia Maria Dozzi Brucki

CARGO/FUNÇÃO: Médica Assistente INSCRIÇÃO CONSELHO REGIONAL N 62.916

PESQUISADOR EXECUTANTE: Patrícia Helena Figueirêdo do Vale Capucho

INSCRIÇÃO CONSELHO REGIONAL No 91418

CARGO/FUNÇÃO: Psicóloga candidata a Pós-Graduação do Departamento de Neurologia

UNIDADE DO HCFMUSP: Divisão de Clínica Neurológica

2. AVALIAÇÃO DO RISCO DA PESQUISA:

$\begin{array}{lll}\text { RISCO MÍNIMO } & X & \text { RISCO MÉDIO } \\ \text { RISCO BAIXO } & \square & \text { RISCO MAIOR }\end{array}$

3.DURAÇÃO DA PESQUISA : 24 meses

Esta pesquisa se destina à adaptação para uso no nosso país, de um teste já utilizado fora do país, para verificar a capacidade que as pessoas têm em tomar decisões necessárias em situações do dia a dia. Este teste chama-se Teste de Julgamento Prático (Test of Practical Judgment TOP-J). Queremos avaliar como idosos saudáveis e pacientes com comprometimento cognitivo leve e até mais avançados, como no caso de demência, respondem a este questionário. Além dele, os participantes serão convidados a responderem a testes que avaliam a capacidade de aprender e guardar novas informações, capacidade de resolução de problemas e de leitura, escrita e capacidade de falar. Os riscos são mínimos e os benefícios desta pesquisa são: fornecer informações sobre a capacidade de julgamento dos pacientes e auxiliar em perícias e investigações clínicas. 
Em qualquer etapa do estudo você poderá ter acesso aos profissionais responsáveis pela pesquisa para esclarecimento de eventuais dúvidas. A principal investigadora é a Dra. Sonia Brucki, que pode ser encontrada nos endereços: Centro de Referência em Distúrbios Cognitivos (CEREDIC) na Rua Arruda Alvim, 206, Cerqueira César, São Paulo/SP, Telefone 3086-1326 ou no Departamento de Neurologia na Av. Dr. Enéas de Carvalho Aguiar, 255, Cerqueira César, São Paulo/SP, Telefone 30697206. Em caso de considerações ou dúvidas sobre a ética da pesquisa, entre em contato com Comitê de Ética em Pesquisa (CEP)Rua Ovídio Pires de Campos, 255- 5॰ andar- tel: 3069-6442 ramais 16, 17, 18 ou 20, FAX: 3069-6442 ramal 26- E-mail: cappesq@hcnet.usp.br.

A participação neste estudo é voluntária e a retirada do consentimento poderá ser feita a qualquer momento, sem qualquer prejuízo à continuidade de seu tratamento na instituição. As informações obtidas serão analisadas em conjunto com as de outros pacientes, não sendo divulgada a identificação de nenhum paciente. Os dados obtidos serão utilizados apenas para esta pesquisa e os participantes serão informados dos resultados obtidos ao final do estudo.

Não há despesas pessoais para o participante em qualquer fase do estudo, incluindo exames e consultas. Também não há compensação financeira relacionada à sua participação. Se existir qualquer despesa adicional ela será absorvida pelo orçamento da pesquisa.

Acredito ter sido suficientemente informado a respeito das informações que li ou que foram lidas para mim, descrevendo o estudo: "Test of Practical Judgment (TOP-J): Adaptação brasileira em amostra de indivíduos cognitivamente saudáveis, com Comprometimento Cognitivo Leve e Demência". Eu discuti com a Dra. Patrícia Helena Figueirêdo do Vale Capucho sobre a minha decisão em participar deste estudo. Ficaram claros para mim quais são os propósitos do estudo, os procedimentos a serem realizados, seus desconfortos e riscos, as garantias de confidencialidade e de esclarecimentos permanentes. Ficou claro também que a minha participação é isenta de despesas e que tenho a garantia do acesso a tratamento hospitalar quando necessário. Concordo voluntariamente em participar deste estudo e poderei retirar o meu consentimento a qualquer momento, antes ou durante o mesmo, sem penalidades, prejuízo ou perda de qualquer benefício que eu possa ter adquirido, ou no meu atendimento neste serviço.

Assinatura do paciente/representante legal Data

1

\section{Assinatura da testemunha}

Data I

para casos de pacientes menores de 18 anos, analfabetos, semi-analfabetos ou portadores de deficiência auditiva ou visual.

(Somente para o responsável do projeto)

Declaro que obtive de forma apropriada e voluntária o Consentimento Livre e Esclarecido deste paciente ou representante legal para a participação neste estudo.

Assinatura do responsável pelo estudo

Data

1


ANEXO C - TOP-J/15 ORIGINAL

PATIENT ID: DATE:
AGE:

GENDER:

\title{
TEST OF PRACTICAL JUDGMENT (TOP-J)
}

\begin{abstract}
DIRECTIONS: Now I would like you to tell me some solutions to everyday problems or
\end{abstract} situations. Please be as complete as possible with your responses.

1. You are vacationing far from home and realize you don't have enough blood pressure pills for the entire trip. What would you do?

\begin{tabular}{|l|l|}
\hline $\mathbf{3}$ & $\begin{array}{l}\text { contact physician (or insurance company) and have prescrip called/faxed into local pharmacy / } \\
\text { call home, have pills Fedexed / call local pharmacy or doctor (Q) - (and have them call in } \\
\text { prescrip) / call local WALMART/CVS, etc. since they have your prescrip records on computer }\end{array}$ \\
\hline $\mathbf{2}$ & $\begin{array}{l}\text { visit local doctor/ER/hospital at vacation site and ask for prescription or describe problem / take } \\
\text { pills to local pharmacy, see if they can help / ("you don't have prescription with you) / have doc or } \\
\text { pharmacy send free samples or prescription / get pharmacy to give you enough pills to make it }\end{array}$ \\
\hline $\mathbf{1}$ & $\begin{array}{l}\text { take smaller doses to make it last longer / ration them / vague response even after query (e.g., "go } \\
\text { to pharmacy \& get some," "call home \& ask for help," "have pharmacist mail them" "get some") }\end{array}$ \\
\hline $\mathbf{0}$ & $\begin{array}{l}\text { wait to see if you need them, no reason to worry in advance / nothing, wait until you get home / } \\
\text { wait until something bad happens / don't know, go without / avoid strenuous activity / }\end{array}$ \\
\hline
\end{tabular}

2. You receive a phone call indicating that you've just won a new car in a contest you entered several months before. The caller asks you to provide your address, social security number, and credit card account. What would you do?

\begin{tabular}{|l|l|}
\hline $\mathbf{3}$ & $\begin{array}{l}\text { do not provide info and ask them to send you a written letter stating terms and conditions of } \\
\text { contest / get verification of authenticity in writing before providing info / provide address so } \\
\text { they can send you info for verification or check with Better Business Bureau/Attorney General }\end{array}$ \\
\hline $\mathbf{2}$ & $\begin{array}{l}\text { hang up / do not provide any info (but fail to seek further information/clarification or vague } \\
\text { reference to gathering more info - e.g., "they need to prove it to me") / not interested / don't } \\
\text { give out info over the phone / try to get ID from them (Q) / ask for their number and call them } \\
\text { back to verify (*contest sponsor is not local) / don't give info but ask questions / notify police (Q) }\end{array}$ \\
\hline $\mathbf{1}$ & $\begin{array}{l}\text { ask for more info over the phone and then provide caller with requested information (some } \\
\text { verification is requested, but not in written form) / just provide address (Q) }\end{array}$ \\
\hline $\mathbf{0}$ & provide info (fail to seek further info or clarification in any way) / just provide address \& SS\# \\
\hline
\end{tabular}

3. You are out of town visiting relatives and suddenly realize that you may have left the stove in your home turned on. What would you do?

\begin{tabular}{|l|l|}
\hline $\mathbf{3}$ & contact someone who could check on your home (e.g., a neighbor, landlord, family member) \\
\hline $\mathbf{2}$ & call police or fire department \\
\hline $\mathbf{1}$ & panic / rush home ( 'you are far from home) \\
\hline $\mathbf{0}$ & do nothing / don't know \\
\hline
\end{tabular}


4. You read a report that the government will reduce monthly social security payments from $\$ 1,000$ to $\$ 500$ for a certain percentage of recipients. What would you do?

\begin{tabular}{|l|l|}
\hline 3 & $\begin{array}{l}\text { find out how likely it is your benefits will be reduced / call to gather more info in attempt to } \\
\text { determine if it affects you / call SS office to find out more (Q) - if you are affected }\end{array}$ \\
\hline $\mathbf{2}$ & $\begin{array}{l}\text { vague attempt at getting more info without directly trying to determine if you are affected or } \\
\text { assumption that you are affected (e.g., "look into it because it's not right" "determine validity } \\
\text { of info" "call \& see what I can do (Q)") / call Senator to get info (Q) / research issue or "find } \\
\text { out why" (without determining if benefits will change) / research how much reduction will be }\end{array}$ \\
\hline $\mathbf{1}$ & $\begin{array}{l}\text { reduce monthly spending / get bills paid so you can budget \$ more closely / go to work / } \\
\text { borrow cash / adjust finances }\end{array}$ \\
\hline $\mathbf{0}$ & $\begin{array}{l}\text { do nothing / wait to see what happens / this doesn't affect me / you can't fight gov (Q) / tell } \\
\text { gov it's a bad idea / complain or call local papers / don't believe it / just live on my resources / } \\
\text { write my senator/congressman and complain / be mad }\end{array}$ \\
\hline
\end{tabular}

5. You are attending a dinner party at your friend's home, but the main dish tastes spoiled. What would you do?

\begin{tabular}{|l|l|}
\hline $\mathbf{3}$ & $\begin{array}{l}\text { stop eating and mention this to your host discreetly / gauge other people's responses and say } \\
\text { something to host (with implication that you would stop eating or that something is wrong) }\end{array}$ \\
\hline $\mathbf{2}$ & $\begin{array}{l}\text { stop eating it (but without telling hostess or other guests) / eat side dishes / cut away bad part / } \\
\text { tell hostess and others "this tastes bad to me, does it taste ok to you?" (Q) / ask host to taste it / } \\
\text { tell other guests aloud (without first mentioning to host) }\end{array}$ \\
\hline $\mathbf{1}$ & $\begin{array}{l}\text { vague response such as push food around plate / gauge other guests' responses (Q) / question } \\
\text { ingredients / lie to others (e.g., "I'm allergic") or deliberately divert attention from food }\end{array}$ \\
\hline $\mathbf{0}$ & keep eating / do nothing - would not want to offend friend / just make do (Q) / just eat some \\
\hline
\end{tabular}

6. You take a daily medication to reduce cholesterol, but a new study printed in your local newspaper suggests that this medication is linked to a higher risk of cancer. What would you do?

\begin{tabular}{|l|l|}
\hline $\mathbf{3}$ & $\begin{array}{l}\text { find out more about the study (e.g., how research was conducted, if other data support finding, } \\
\text { what kind of cancer) / call an expert / call your physician / inquire about alternative meds }\end{array}$ \\
\hline $\mathbf{2}$ & $\begin{array}{l}\text { passive attempt to discern validity of study (e.g., "wait for more confirmation" / "ignore it until } \\
\text { it's verified by another study") }\end{array}$ \\
\hline $\mathbf{1}$ & $\begin{array}{l}\text { stop taking med and then gather more info from doc or medline/ stop taking medication / } \\
\text { mention to doctor \& insist on stopping medication / continue taking med (with good rationale) }\end{array}$ \\
\hline $\mathbf{0}$ & $\begin{array}{l}\text { ignore the findings / do nothing / laugh at the publication - it's impossible / continue to take } \\
\text { the medication (with bad rationale - i.e., "I'm old so who cares if I get cancer") }\end{array}$ \\
\hline
\end{tabular}


7. You begin to have trouble driving due to increasing night blindness and mild confusion. What would you do?

\begin{tabular}{|l|l|}
\hline $\mathbf{3}$ & $\begin{array}{l}\text { consult professional such as physician or driving evaluation to determine cause of problem } \\
\text { (and stop driving at night until cause is determined / while awaiting doctor's opinion) }\end{array}$ \\
\hline $\mathbf{2}$ & $\begin{array}{l}\text { avoid night driving / only drive to places you know well / adjust driving patterns (Q) / } \\
\text { have spouse or friend drive at night / vague reference to finding out more about problem such } \\
\text { as "see doctor" (Q) or "have my wife check me out and let her do more of the driving" }\end{array}$ \\
\hline $\mathbf{1}$ & $\begin{array}{l}\text { give up driving altogether / quit driving (without mention of being evaluated) / discuss with } \\
\text { family and give up driving / "I don't drive" (Q) / get someone to drive for me / "safety comes } \\
\text { first, but I'm not sure" }\end{array}$ \\
\hline $\mathbf{0}$ & do nothing / "don't go out at night" (Q) / only go out if it's not raining \\
\hline
\end{tabular}

8. You find a wallet on the sidewalk. You pick it up and 5 steps later you find a $\$ 50$ bill. What would you do?

$3 \quad$ any answer which seeks to determine ownership of or return both wallet and $\$ 50$ bill and recognizes possibility they don't belong to same person / take wallet to the police or town hall and explain about bill / look for ID and call person to verify amount in wallet (and return both)

2 any answer which seeks to determine owner/return wallet but does not note $\$ 50$ as possibly being separate from wallet (e.g., "look for ID in wallet and try to find out who it belongs to") / take "it" to police / any response which assumes the wallet and bill go together and should both be returned to single owner / turn "it" or "both" in to police

1 take wallet to the police station but keep the bill / "give back wallet, if I like the person give back the bill, otherwise keep it" / keep bill but try to return wallet

0 keep both / just keep walking / "I would pick it up and run like hell - thinking it was a trap"

9. You drive to a mall in a nearby city, and by the time you finish shopping it is dark outside. Your car is parked a great distance from the exit and there have recently been several muggings in the area. What would you do?

3 find security or police and ask them to accompany you to your car / gauge safety of the situation and get someone to walk you to car or bring car to you / (*you went to the mall alone)

2 call family or friend to pick you up / walk alongside other patron(s) / call a taxi

1 go to car but watch for anything suspicious / walk to car \& hold key in hand as protective device / walk to car but keep eye out for security patrol / walk to car but keep cell phone close

$\mathbf{0} \quad$ "walk to car" (with no recognition that situation may be unsafe) / "I'm not worried about other people attacking" / don't know / panic / this would never happen to me / "I'm not concerned" / it's a bad idea to go through an area like that / scream and holler and hit them with something 
10. You meet two close friends for dinner at a new restaurant you've been excited about trying. Upon arrival, however, the manager explains that one of your friends cannot enter because he/she is dressed too casually. What would you do?

\begin{tabular}{|l|l|}
\hline $\mathbf{3}$ & $\begin{array}{l}\text { go somewhere else / find a different restaurant that permits informal attire and plan to visit new } \\
\text { restaurant another time / eat at restaurant bar in casual attire / see if your friend has other } \\
\text { clothes with them or loaner in restaurant (Q) ("your friend is wearing jeans and sneakers) }\end{array}$ \\
\hline $\mathbf{2}$ & $\begin{array}{l}\text { vague response indicating "you" would "leave" without specifying going to another restaurant } \\
\text { or who is included (e.g., "I would go to McDonalds") / find out what it would take to make } \\
\text { dress appropriate (Q) / extreme reaction "get very angry/leave \& never return" / vague } \\
\text { response such as "I would discuss it with my friend and let him decide" / discuss with manager }\end{array}$ \\
\hline $\mathbf{1}$ & $\begin{array}{l}\text { tell casually dressed friend to go home and change and return to the restaurant (you will be } \\
\text { waiting at restaurant bar) ("your friend lives far away) / find a nearby store and purchase } \\
\text { something appropriate / attempt to convince or bribe manager to let you all in/break rule / } \\
\text { reschedule for a different night altogether }\end{array}$ \\
\hline $\mathbf{0}$ & enter with appropriately dressed friend and leave informally dressed friend behind \\
\hline
\end{tabular}

11. You are driving along the highway in the left lane at approximately the speed limit. You notice that the car behind you is tailgating. What would you do?

3 signal and switch lanes / allow car to pass (Q) / move over one lane / pull over and allow car to pass or get ahead (with implication of switching lanes, but not getting off road entirely) / answer that accomplishes goal of getting away from driver with little effort, consequence, or risk - and does not involve physically getting off the road (*it is a 4-lane highway)

2 let them pass and call 911 or call for help / speed up and then signal to let car pass / (slow down or speed up with implication of letting car pass) and pull over to side of road or off ramp

1 an answer that accomplishes getting away from tailgater but seems extreme or does not involve letting tailgating car pass or is dangerous / (get nervous and) get off at next exit / drive to police station if car seems to be following you / call 911 / pull off at closest available spot / "do anything to try to lose them" / wave them past you (Q) / go slow until he passes / hit breaks momentarily to give him a lesson, then let him pass / you shouldn't drive in the left lane (Q)

$\mathbf{0}$ do nothing / keep going at my current speed, don't worry / continue at my pace / speed up / slam on brakes / tap breaks / speed up then slow down / box them in with car next to you / put on "flashers" (to make him think you're stepping on brakes) then slow down / open window and wave him back / just keep going until you reach exit / "leave enough space so they won't hit me if I have to stop" 
12. You see a small, friendly dog with a collar alone on the sidewalk in your neighborhood. What would you do?

\begin{tabular}{|l|l|}
\hline $\mathbf{3}$ & $\begin{array}{l}\text { (try to determine if dog is safe) -- then look at info on collar and look around for owner or call } \\
\text { number listed on the tag / check collar \& call local pound or animal shelter / check collar to } \\
\text { find owner / if you are afraid of dogs, call the humane center or call for help }\end{array}$ \\
\hline $\mathbf{2}$ & $\begin{array}{l}\text { look at collar (without statement about locating ID or finding owner) / vague response that } \\
\text { attempts to locate owner ("try to identify dog \& call owner"(Q)) without mention of looking at } \\
\text { collar or tag / call dog office or town (Q) }\end{array}$ \\
\hline $\mathbf{1}$ & $\begin{array}{l}\text { an answer that involves physically trying to locate owner without clear plan or without looking } \\
\text { at collar (e.g., "ask patrons in nearby store or near you on street if they lost a dog" / pick it up } \\
\text { and go door-to-door / take it home \& advertise / pick it up and bring it to vet / }\end{array}$ \\
\hline $\mathbf{0}$ & nothing, keep walking / leave it alone / say "hello" to dog or talk to dog and walk on \\
\hline
\end{tabular}

13. You are diagnosed with a rare blood disease causing severe discomfort and cognitive deterioration. Your doctor explains that a new medical procedure can help cure the disease, but this procedure has a $10 \%$ mortality rate. Alternatively, a commonly used medication can provide relief of some symptoms. What would you do?

\begin{tabular}{|l|l|}
\hline $\mathbf{3}$ & $\begin{array}{l}\text { research issue \& carefully consider risks and benefits -- and then decide / find out about } \\
\text { alternative treatments / ask a lot of questions (Q) / get second opinion before deciding / get } \\
\text { temporary relief and then a second opinion }\end{array}$ \\
\hline $\mathbf{2}$ & $\begin{array}{l}\text { chooses one tx with good rationale / chooses one tx but recognizes that other treatment may be } \\
\text { viable as other option / try med first and if it doesn't work, try cure / consideration of } \\
\text { alternatives but chooses one without more research / follow doctor's advice (and take med) / } \\
\text { "inquire about side effects" (without addressing need to make a decision) }\end{array}$ \\
\hline $\mathbf{1}$ & $\begin{array}{l}\text { immediately chooses one option or the other without rationale (e.g., "try medication" or "have } \\
\text { operation") / vague response "opt for safer treatment" (Q) / "go for the help I could get" (Q) / } \\
\text { "what is least costly to relatives" / get advice from relatives / find out what to do to get well }\end{array}$ \\
\hline $\mathbf{0}$ & don't know / do nothing \\
\hline
\end{tabular}

14. You realize that you accidentally scheduled two social engagements (on the opposite ends of town) for the same afternoon. What would you do?

\begin{tabular}{|l|l|}
\hline $\mathbf{3}$ & $\begin{array}{l}\text { call to reschedule one of them (possibly the less important or more flexible one) and apologize } \\
\text { / cancel one / call to explain (Q) (*the engagements are approximately 40 miles apart) }\end{array}$ \\
\hline $\mathbf{2}$ & $\begin{array}{l}\text { try to fulfill both by cutting each short / vague response (e.g., "call both and gauge reactions } \\
\text { and then decide") / go to closest one then leave and go to other }\end{array}$ \\
\hline $\mathbf{1}$ & just go to one - ignore the other \\
\hline $\mathbf{0}$ & don't go to either - too overwhelming / cancel both / give my tickets away to someone else (Q) \\
\hline
\end{tabular}


15. Your trusted financial advisor tells you that stocks will significantly decline in value over the next several years while bonds will rise in value. You currently own stocks but no bonds and have the opportunity to change your investment portfolio based on this advice. What would you do?

\begin{tabular}{|l|l|}
\hline $\mathbf{3}$ & $\begin{array}{l}\text { sell a selected amount of stocks and buy a selected amount of bonds in order to follow the } \\
\text { recommendation but avoid too drastic a change / response that involves shifting your portfolio } \\
\text { in some way / seek advice of others and then make a change ("other advisors agree) }\end{array}$ \\
\hline $\mathbf{2}$ & $\begin{array}{l}\text { vague response "go with the advice because I trust him" "change it into bonds" "try to better } \\
\text { yourself financially" (Q) / seek further advice / research it / "don't blindly take advice, use own } \\
\text { judgment" (Q) / wait until you see evidence of movement in bond market before deciding }\end{array}$ \\
\hline $\mathbf{1}$ & $\begin{array}{l}\text { drastic change based on this advice (e.g., sell all stocks and use money to buy bonds / get rid of } \\
\text { stocks but don't buy any bonds - put it all in a money market or CDs or cash / sell all stocks } \\
\text { and get out of market) / no change with rationale (e.g., do nothing since you can't tell what will } \\
\text { happen next, "I would consult someone else but in the end stick with my stock") }\end{array}$ \\
\hline $\mathbf{0}$ & don't know / don't understand anything about that / "nothing" \\
\hline
\end{tabular}

SCORING: The score is the total of all 15 items $(\max =45)$

TOTAL SCORE $=$

\section{ADDITIONAL INFORMATION FOR ADMINISTRATION AND SCORING:}

- When necessary, repeat the question once to ensure the participant's understanding.

- Record the participant's responses verbatim.

- Score as you go, and be SURE to query when necessary: Query when indicated by $(Q)$ - with the statement "Tell me more about that" or "Explain what you mean."

- If, following query, the participant provides additional information that raises the response value, credit him/her with the higher value.

- Additional information (in bold) can be provided if the participant directly requests this information or gives a response that contradicts this information.

- When reading question 10, gender of friend should be specified as that of the participant.

- If the participant gives two or more responses, ask him/her directly to choose among them: "You stated $x, y$, and $z$, -- which is your final response?"

- If the participant gives several responses even after querying, go with response you feel best captures the intended response.

- If the participant says "this does not apply to me" (e.g., "I don't drive anymore") - ask him/her to imagine that he/she does (e.g., "For this question, please imagine that you do still drive"). If participant is still unwilling or unable to perspective-take, credit him/her with a "0" response.

- As a general rule, if the participant gives a response which has features of a 3-point score but is vague, give credit for a 2 -point score. 


\section{ANEXO D - TOP-J/9 ORIGINAL}

\section{TEST OF PRACTICAL JUDGMENT (TOP-J)}

DIRECTIONS: Now I would like you to tell me some solutions to everyday problems or
situations. Please be as complete as possible with your responses.

1. You are vacationing far from home and realize you don't have enough blood pressure pills for the entire trip. What would you do?

\begin{tabular}{|l|l|}
\hline $\mathbf{3}$ & $\begin{array}{l}\text { contact physician (or insurance company) and have prescrip called/faxed into local pharmacy / } \\
\text { call home, have pills Fedexed / call local pharmacy or doctor (Q) - (and have them call in } \\
\text { prescrip) / call local WALMART/CVS, etc. since they have your prescrip records on computer }\end{array}$ \\
\hline $\mathbf{2}$ & $\begin{array}{l}\text { visit local doctor/ER/hospital at vacation site and ask for prescription or describe problem / take } \\
\text { pills to local pharmacy, see if they can help / ("you don't have prescription with you) / have doc or } \\
\text { pharmacy send free samples or prescription / get pharmacy to give you enough pills to make it }\end{array}$ \\
\hline $\mathbf{1}$ & $\begin{array}{l}\text { take smaller doses to make it last longer / ration them / vague response even after query (e.g., "go } \\
\text { to pharmacy \& get some," "call home \& ask for help," "have pharmacist mail them" "get some") }\end{array}$ \\
\hline $\mathbf{0}$ & $\begin{array}{l}\text { wait to see if you need them, no reason to worry in advance / nothing, wait until you get home / } \\
\text { wait until something bad happens / don't know, go without / avoid strenuous activity / }\end{array}$ \\
\hline
\end{tabular}

2. You receive a phone call indicating that you've just won a new car in a contest you entered several months before. The caller asks you to provide your address, social security number, and credit card account. What would you do?

\begin{tabular}{|l|l|}
\hline $\mathbf{3}$ & $\begin{array}{l}\text { do not provide info and ask them to send you a written letter stating terms and conditions of } \\
\text { contest / get verification of authenticity in writing before providing info / provide address so } \\
\text { they can send you info for verification or check with Better Business Bureau/Attorney General }\end{array}$ \\
\hline $\mathbf{2}$ & $\begin{array}{l}\text { hang up / do not provide any info (but fail to seek further information/clarification or vague } \\
\text { reference to gathering more info - e.g., "they need to prove it to me") / not interested / don't } \\
\text { give out info over the phone / try to get ID from them (Q) / ask for their number and call them } \\
\text { back to verify (*contest sponsor is not local) / don't give info but ask questions / notify police (Q) }\end{array}$ \\
\hline $\mathbf{1}$ & $\begin{array}{l}\text { ask for more info over the phone and then provide caller with requested information (some } \\
\text { verification is requested, but not in written form) / just provide address (Q) }\end{array}$ \\
\hline $\mathbf{0}$ & provide info (fail to seek further info or clarification in any way) / just provide address \& SS\# \\
\hline
\end{tabular}


3. You are out of town visiting relatives and suddenly realize that you may have left the stove in your home turned on. What would you do?

\begin{tabular}{|l|l|}
\hline $\mathbf{3}$ & contact someone who could check on your home (e.g., a neighbor, landlord, family member) \\
\hline $\mathbf{2}$ & call police or fire department \\
\hline $\mathbf{1}$ & panic / rush home (*you are far from home $)$ \\
\hline $\mathbf{0}$ & do nothing / don’t know \\
\hline
\end{tabular}

4. You read a report that the government will reduce monthly social security payments from $\$ 1,000$ to $\$ 500$ for a certain percentage of recipients. What would you do?

\begin{tabular}{|l|l|}
\hline $\mathbf{3}$ & $\begin{array}{l}\text { find out how likely it is your benefits will be reduced / call to gather more info in attempt to } \\
\text { determine if it affects you / call SS office to find out more (Q) - if you are affected }\end{array}$ \\
\hline $\mathbf{2}$ & $\begin{array}{l}\text { vague attempt at getting more info without directly trying to determine if you are affected or } \\
\text { assumption that you are affected (e.g., "look into it because it's not right" "determine validity } \\
\text { of info" "call \& see what I can do (Q)") / call Senator to get info (Q) / research issue or "find } \\
\text { out why" (without determining if benefits will change) / research how much reduction will be }\end{array}$ \\
\hline $\mathbf{1}$ & $\begin{array}{l}\text { reduce monthly spending / get bills paid so you can budget \$ more closely / go to work / } \\
\text { borrow cash / adjust finances }\end{array}$ \\
\hline $\mathbf{0}$ & $\begin{array}{l}\text { do nothing / wait to see what happens / this doesn't affect me / you can't fight gov (Q) / tell } \\
\text { gov it's a bad idea / complain or call local papers / don't believe it / just live on my resources / } \\
\text { write my senator/congressman and complain / be mad }\end{array}$ \\
\hline
\end{tabular}

5. You take a daily medication to reduce cholesterol, but a new study printed in your local newspaper suggests that this medication is linked to a higher risk of cancer. What would you do?

\begin{tabular}{|l|l|}
\hline $\mathbf{3}$ & $\begin{array}{l}\text { find out more about the study (e.g., how research was conducted, if other data support finding, } \\
\text { what kind of cancer) / call an expert / call your physician / inquire about alternative meds }\end{array}$ \\
\hline $\mathbf{2}$ & $\begin{array}{l}\text { passive attempt to discern validity of study (e.g., "wait for more confirmation" / "ignore it until } \\
\text { it's verified by another study") }\end{array}$ \\
\hline $\mathbf{1}$ & $\begin{array}{l}\text { stop taking med and then gather more info from doc or medline/ stop taking medication / } \\
\text { mention to doctor \& insist on stopping medication / continue taking med (with good rationale) }\end{array}$ \\
\hline $\mathbf{0}$ & $\begin{array}{l}\text { ignore the findings / do nothing / laugh at the publication - it's impossible / continue to take } \\
\text { the medication (with bad rationale - i.e., "I'm old so who cares if I get cancer") }\end{array}$ \\
\hline
\end{tabular}


6. You begin to have trouble driving due to increasing night blindness and mild confusion. What would you do?

\begin{tabular}{|l|l|}
\hline $\mathbf{3}$ & $\begin{array}{l}\text { consult professional such as physician or driving evaluation to determine cause of problem } \\
\text { (and stop driving at night until cause is determined / while awaiting doctor's opinion) }\end{array}$ \\
\hline $\mathbf{2}$ & $\begin{array}{l}\text { avoid night driving / only drive to places you know well / adjust driving patterns (Q) / } \\
\text { have spouse or friend drive at night / vague reference to finding out more about problem such } \\
\text { as "see doctor" (Q) or "have my wife check me out and let her do more of the driving" }\end{array}$ \\
\hline $\mathbf{1}$ & $\begin{array}{l}\text { give up driving altogether / quit driving (without mention of being evaluated) / discuss with } \\
\text { family and give up driving / "I don't drive" (Q) / get someone to drive for me / "safety comes } \\
\text { first, but I'm not sure" }\end{array}$ \\
\hline $\mathbf{0}$ & do nothing / "don't go out at night" (Q) / only go out if it's not raining \\
\hline
\end{tabular}

7. You find a wallet on the sidewalk. You pick it up and 5 steps later you find a $\$ 50$ bill. What would you do?

\begin{tabular}{|l|l|}
\hline $\mathbf{3}$ & $\begin{array}{l}\text { any answer which seeks to determine ownership of or return both wallet and \$50 bill and } \\
\text { recognizes possibility they don't belong to same person / take wallet to the police or town hall } \\
\text { and explain about bill / look for ID and call person to verify amount in wallet (and return both) }\end{array}$ \\
\hline $\mathbf{2}$ & $\begin{array}{l}\text { any answer which seeks to determine owner/return wallet but does not note \$50 as possibly } \\
\text { being separate from wallet (e.g. "look for ID in wallet and try to find out who it belongs to") / } \\
\text { take "it" to police / any response which assumes the wallet and bill go together and should both } \\
\text { be returned to single owner / turn "it" or "both" in to police }\end{array}$ \\
\hline $\mathbf{1}$ & $\begin{array}{l}\text { take wallet to the police station but keep the bill / "give back wallet, if I like the person give } \\
\text { back the bill, otherwise keep it" / keep bill but try to return wallet }\end{array}$ \\
\hline $\mathbf{0}$ & keep both / just keep walking / "I would pick it up and run like hell - thinking it was a trap" \\
\hline
\end{tabular}

8. You see a small, friendly dog with a collar alone on the sidewalk in your neighborhood. What would you do?

\begin{tabular}{|l|l|}
\hline $\mathbf{3}$ & $\begin{array}{l}\text { (try to determine if dog is safe) -- then look at info on collar and look around for owner or call } \\
\text { number listed on the tag / check collar \& call local pound or animal shelter / check collar to } \\
\text { find owner / if you are afraid of dogs, call the humane center or call for help }\end{array}$ \\
\hline $\mathbf{2}$ & $\begin{array}{l}\text { look at collar (without statement about locating ID or finding owner) / vague response that } \\
\text { attempts to locate owner ("try to identify dog \& call owner"(Q)) without mention of looking at } \\
\text { collar or tag / call dog office or town (Q) }\end{array}$ \\
\hline $\mathbf{1}$ & $\begin{array}{l}\text { an answer that involves physically trying to locate owner without clear plan or without looking } \\
\text { at collar (e.g., "ask patrons in nearby store or near you on street if they lost a dog" / pick it up } \\
\text { and go door-to-door / take it home \& advertise / pick it up and bring it to vet / }\end{array}$ \\
\hline $\mathbf{0}$ & nothing, keep walking / leave it alone / say "hello" to dog or talk to dog and walk on \\
\hline
\end{tabular}


9. Your trusted financial advisor tells you that stocks will significantly decline in value over the next several years while bonds will rise in value. You currently own stocks but no bonds and have the opportunity to change your investment portfolio based on this advice. What would you do?

\begin{tabular}{|l|l|}
\hline $\mathbf{3}$ & $\begin{array}{l}\text { sell a selected amount of stocks and buy a selected amount of bonds in order to follow the } \\
\text { recommendation but avoid too drastic a change / response that involves shifting your portfolio } \\
\text { in some way / seek advice of others and then make a change ("other advisors agree) }\end{array}$ \\
\hline $\mathbf{2}$ & $\begin{array}{l}\text { vague response "go with the advice because I trust him" "change it into bonds" "try to better } \\
\text { yourself financially" (Q) / seek further advice / research it / "don't blindly take advice, use own } \\
\text { judgment" (Q) / wait until you see evidence of movement in bond market before deciding }\end{array}$ \\
\hline $\mathbf{1}$ & $\begin{array}{l}\text { drastic change based on this advice (e.g., sell all stocks and use money to buy bonds / get rid of } \\
\text { stocks but don't buy any bonds - put it all in a money market or CDs or cash / sell all stocks } \\
\text { and get out of market) / no change with rationale (e.g., do nothing since you can't tell what will } \\
\text { happen next, "I would consult someone else but in the end stick with my stock") }\end{array}$ \\
\hline $\mathbf{0}$ & don't know / don't understand anything about that / "nothing" \\
\hline
\end{tabular}

SCORING: The score is the total of all 9 items $(\max =27)$

TOTAL SCORE $=$

\section{ADDITIONAL INFORMATION FOR ADMINISTRATION AND SCORING:}

- When necessary, repeat the question once to ensure the participant's understanding.

- Record the participant's responses verbatim.

- Score as you go, and be SURE to query when necessary: Query when indicated by (Q) - with the statement "Tell me more about that" or "Explain what you mean."

- If, following query, the participant provides additional information that raises the response value, credit him/her with the higher value.

- Additional information (in bold) can be provided if the participant directly requests this information or gives a response that contradicts this information.

- If the participant gives two or more responses, ask him/her directly to choose among them: "You stated $x, y$, and $z,--$ which is your final response?"

- If the participant gives several responses even after querying, go with response you feel best captures the intended response.

- If the participant says "this does not apply to me" (e.g., "I don't drive anymore") - ask him/her to imagine that he/she does (e.g., "For this question, please imagine that you do still drive"). If participant is still unwilling or unable to perspective-take, credit him/her with a "0" response.

- As a general rule, if the participant gives a response which has features of a 3-point score but is vague, give credit for a 2 -point score. 


\section{ANEXO E - MODIFICAÇÃO 1}

Nome:

Idade:

Data:

Sexo:

\section{Teste de Julgamento Prático (TOP-J)}

INSTRUCÕES: Agora eu gostaria que você me falasse algumas soluções para problemas ou situações cotidianos. Por favor, seja o mais claro possível em suas respostas.

1. Você está de férias longe de casa e percebe que não tem comprimidos controlados suficientes para toda a viagem. O que você faria?

\begin{tabular}{|l|l|}
\hline 3 & $\begin{array}{l}\text { procura um médico (ou plano de saúde) e pega uma receita por telefone ou fax em } \\
\text { uma farmácia local/ liga para casa e pede para alguém mandar os remédios Sedex/ } \\
\text { liga para um médico ou farmácia local (Q) (e consegue uma receita)/ liga para a } \\
\text { farmácia onde você costuma comprar e pergunta se eles tem o histórico das suas } \\
\text { receitas no computador }\end{array}$ \\
\hline 2 & $\begin{array}{l}\text { vai ao médico local/Pronto Socorro/hospital local e pede uma receita ou descreve o } \\
\text { problema/ leva os remédios a uma farmácia local e vê se eles conseguem ajudar/ } \\
\text { ("você nâo tem a receita com você)/ pega amostra grátis ou receita em um médico } \\
\text { ou farmácia/ convence o farmacêutico a te dar pílulas suficientes }\end{array}$ \\
\hline $\mathbf{1}$ & $\begin{array}{l}\text { toma doses menores para durarem mais/ economiza-os (raciona-os)/ respostas } \\
\text { vagas mesmo após questionado (ex. "vou à farmácia e pego", "ligo para casa e } \\
\text { peço ajuda", "consigo que o farmacêutico envie por e-mail", "consigo algum") }\end{array}$ \\
\hline $\mathbf{0}$ & $\begin{array}{l}\text { espera para ver se você precisa deles, não há motivo para se preocupar adiantado/ } \\
\text { nada, espero até chegar em casa/ espera até alguma coisa ruim acontecer/ não sei, } \\
\text { continuo sem/ evito esforço fisico }\end{array}$ \\
\hline
\end{tabular}

2. Você recebe um telefonema avisando que acabou de ganhar um carro em um sorteio do qual participou muitos meses antes. A pessoa que ligou pede para você fornecer seu endereço, CPF e número do cartão de crédito. O que você faria?

\begin{tabular}{|l|l|}
\hline 3 & $\begin{array}{l}\text { não fornece informações e pede para enviarem um documento por escrito } \\
\text { declarando termos e condições do sorteio/ pede uma comprovação da autenticidade } \\
\text { por escrito antes de fornecer informações/ fornece o endereço para que possam } \\
\text { enviar-lhe comprovação ou checa com o Serviço de Proteção ao Consumidor }\end{array}$ \\
\hline 2 & $\begin{array}{l}\text { desliga/ não fornece informação alguma (mas não solicita informações adicionais/ } \\
\text { esclarecimento ou vaga referência sobre reunir mais informações - ex., "eles }\end{array}$ \\
\hline
\end{tabular}




\begin{tabular}{|l|l|}
\hline & $\begin{array}{l}\text { necessitam provar isso para mim")/ não interessado/ não fornece informações por } \\
\text { telefone/ tento pegar identificação deles (Q)/ pede o número deles e liga de volta } \\
\text { para conferir (*o patrocinador do sorteio não é local)/ não dá informações mas } \\
\text { faz perguntas/ avisa a policia (Q) }\end{array}$ \\
\hline $\mathbf{1}$ & $\begin{array}{l}\text { pede mais informações por telefone e depois fornece as informações solicitadas } \\
\text { (alguma comprovação é pedida, mas não por escrito)/ somente fornece o endereço } \\
(\mathbf{Q})\end{array}$ \\
\hline $\mathbf{0}$ & $\begin{array}{l}\text { fornece informações (não solicita informações ou esclarecimentos posteriores de } \\
\text { forma alguma)/ somente fornece endereço e CPF }\end{array}$ \\
\hline
\end{tabular}

3. Você está fora da cidade visitando parentes e de repente lembra que pode ter deixado o fogão da sua casa ligado. 0 que você faria?

\begin{tabular}{|l|l|}
\hline $\mathbf{3}$ & $\begin{array}{l}\text { entra em contato com alguém que possa ir na sua casa checar (ex. vizinho, } \\
\text { proprietário, membro da familia) }\end{array}$ \\
\hline $\mathbf{2}$ & liga para a polícia ou bombeiro \\
\hline $\mathbf{1}$ & pânico/ corre para casa ( ${ }^{\star}$ você está longe de casa) \\
\hline $\mathbf{0}$ & faz nada/ não sabe \\
\hline
\end{tabular}

4. Você leu uma notícia de que o governo vai reduzir mensalmente os pagamentos do INSS de $\mathbf{R} \$ 1500,00$ para $\mathbf{R} \$ 750,00$ para uma determinada porcentagem de pensionistas. $\underline{\mathrm{O} \text { que você faria? }}$

\begin{tabular}{|l|l|}
\hline 3 & $\begin{array}{l}\text { tenta descobrir a probabilidade dos seus beneficios serem reduzidos/liga para obter } \\
\text { mais informações na tentativa de determinar se isso afeta você/ ligar para o INSS } \\
\text { para saber mais (Q) - se você será afetado }\end{array}$ \\
\hline $\mathbf{2}$ & $\begin{array}{l}\text { Tenta vagamente obter mais informações sem tentar determinar diretamente se } \\
\text { você será afetado ou supor que será afetado (ex. "eu verifico, porque isso não está } \\
\text { certo" "determino a validade da informação" "ligo e vejo o que posso fazer (Q)"/ } \\
\text { liga para o senador para obter informação (Q)/ pesquisa o problema ou "procura o } \\
\text { motivo" (sem averiguar se os beneficios irão mudar)/ pesquisa quanto de redução } \\
\text { haverá }\end{array}$ \\
\hline $\mathbf{1}$ & $\begin{array}{l}\text { reduz os gastos mensais/ deixa as contas pagas para manter o orçamento } \\
\text { controlado/ vai trabalhar/ pede dinheiro emprestado/ ajusta as finanças }\end{array}$ \\
\hline $\mathbf{0}$ & $\begin{array}{l}\text { faz nada/ espera para ver o que vai acontecer/ isso não me afeta/ não se pode brigar } \\
\text { com o governo (Q)/ diz para o governo que isso é uma má idéia/ reclama ou liga } \\
\text { para jornais locais/não acredito nisso/ eu só vivo com meus recursos/ escrevo para } \\
\text { o meu senador/deputado e me queixo/ fico bravo }\end{array}$ \\
\hline
\end{tabular}


5. Você está em um jantar na casa de um amigo, mas o prato principal está com gosto de estragado. O que você faria?

\begin{tabular}{|l|l|}
\hline 3 & $\begin{array}{l}\text { para de comer e avisa o anfitrião discretamente/ avalia a reação das pessoas e diz } \\
\text { alguma coisa para o anfitrião (querendo dizer que é melhor parar de comer ou que } \\
\text { algo está errado) }\end{array}$ \\
\hline 2 & $\begin{array}{l}\text { para de comer (mas sem falar para o anfitrião ou outros convidados)/ come os } \\
\text { acompanhamentos/ separa a parte ruim/ fala para o anfitrião e para os outros "isto } \\
\text { está com o gosto ruim para mim, está bom para vocês?" (Q)/ pede para o anfitrião } \\
\text { provar/ fala alto para os outros convidados (sem falar primeiro para o anfitrião) }\end{array}$ \\
\hline $\mathbf{1}$ & $\begin{array}{l}\text { respostas vagas como empurrar a comida ao redor do prato/ outras respostas vagas } \\
\text { (Q)/ pergunta sobre os ingredientes/ mente para os outros (ex. "Eu sou alérgico") } \\
\text { ou propositalmente desvia a atenção da comida }\end{array}$ \\
\hline $\mathbf{0}$ & $\begin{array}{l}\text { continua comendo/ faz nada - não queria ofender um amigo/ se vira com o que for } \\
\text { possivel (Q)/ somente come um pouco }\end{array}$ \\
\hline
\end{tabular}

6. Você toma uma medicação diária para reduzir o colesterol, mas um estudo novo publicado no jornal local sugere que esta medicação está relacionada a um alto risco para câncer. $\underline{O}$ que você faria?

\begin{tabular}{|l|l|}
\hline 3 & $\begin{array}{l}\text { procura saber mais sobre o estudo (ex. como a pesquisa foi conduzida, se outros } \\
\text { dados apóiam os achados, que tipo de câncer)/ liga para um especialista/ liga para } \\
\text { seu médico/ pergunta sobre medicamentos alternativos }\end{array}$ \\
\hline $\mathbf{2}$ & $\begin{array}{l}\text { tenta passivamente discernir a validade do estudo (ex. "espero por mais } \\
\text { confirmações"/ "ignoro isso até que seja verificado por outro estudo") }\end{array}$ \\
\hline $\mathbf{1}$ & $\begin{array}{l}\text { para de tomar medicação e depois obtém maiores informações do médico ou sites } \\
\text { médicos/ para de tomar a medicação/ fala para o médico e insiste em parar de } \\
\text { tomar a medicação/ continua tomando a medicação (com boa justificativa) }\end{array}$ \\
\hline $\mathbf{0}$ & $\begin{array}{l}\text { ignora os achados/ faz nada/ ri da publicação - isso é impossível/ continua } \\
\text { tomando a medicação (com justificativa ruim - ex. "Eu sou velho, quem se importa } \\
\text { se eu tiver câncer") }\end{array}$ \\
\hline
\end{tabular}

7. Você está começando a ter problemas para dirigir devido a problemas na visão noturna e discreta confusão mental. O que você faria?

3 Faz uma consulta com um profissional, como um médico ou avaliação de direção para determinar a causa do problema (e para de dirigir a noite até que a causa seja 


\begin{tabular}{|l|l|}
\hline 2 & determinada/ enquanto espera a opinião do médico) \\
\hline 2 & $\begin{array}{l}\text { evita dirigir a noite/ somente dirige para lugares que você conhece bem/ ajusta os } \\
\text { modelos de direção (Q)/ pede para esposa ou amigo dirigir a noite/ vaga referência } \\
\text { sobre saber mais sobre o problema como "ir ao médico" (Q) ou "peço para minha } \\
\text { esposa me supervisionar e deixo ela dirigir mais" }\end{array}$ \\
\hline $\mathbf{1}$ & $\begin{array}{l}\text { desiste completamente de dirigir/ deixar de dirigir (sem menção de estar sendo } \\
\text { avaliado)/ discutir com a familia e desistir de dirigir/ "Eu não dirijo" (Q)/ ter } \\
\text { alguém para dirigir para mim/ "segurança vem primeiro, mas eu não tenho certeza" }\end{array}$ \\
\hline $\mathbf{0}$ & faz nada/ "não saio de casa a noite" (Q)/ saio somente se não estiver chovendo \\
\hline
\end{tabular}

8. Você acha uma carteira na calçada. Você a pega e 5 passos depois você acha uma nota de $\mathrm{R} \$ 50,00$. O que você faria?

\begin{tabular}{|l|l|}
\hline 3 & $\begin{array}{l}\text { qualquer resposta que busque determinar quem é o dono ou devolver ambos a } \\
\text { carteira e o dinheiro e reconhecer a possibilidade de que não pertençam à mesma } \\
\text { pessoa/ levar a carteira à polícia ou prefeitura e explicar sobre a nota/ procurar o } \\
\text { RG na carteira e ligar para a pessoa para verificar a quantia na carteira (e devolver } \\
\text { ambos) }\end{array}$ \\
\hline 2 & $\begin{array}{l}\text { qualquer resposta que busque tentar determinar o dono/ devolver a carteira mas não } \\
\text { a nota que possivelmente não estava na carteira (ex. procurar o RG na carteira e } \\
\text { tentar encontrar o dono)/ levá-la à polícia/ qualquer resposta dizendo que a carteira } \\
\text { e a nota deveriam ser devolvidas a um único dono/ leva a carteira ou ambos à } \\
\text { polícia }\end{array}$ \\
\hline $\mathbf{1}$ & $\begin{array}{l}\text { leva a carteira para a delegacia mas fica com a nota/ "devolvo a carteira, e se eu } \\
\text { gostar da pessoa devolvo a nota, do contrário fico com ela"/ fica com a nota mas } \\
\text { tenta devolver a carteira }\end{array}$ \\
\hline $\mathbf{0}$ & $\begin{array}{l}\text { fica com ambos/ somente continua andando/ "eu pegaria e sairia correndo - } \\
\text { achando que é uma armadilha" }\end{array}$ \\
\hline
\end{tabular}

9. Você foi dirigindo a um shopping em uma cidade vizinha, e quando acabou de fazer as compras estava escuro lá fora. Seu carro está parado a uma grande distância da saída e lá tem acontecido muitos assaltos recentemente. $\underline{O}$ que você faria?

\begin{tabular}{|l|l|}
\hline 3 & $\begin{array}{l}\text { acha um segurança ou policial e pede a eles para acompanhá-lo ao seu carro/ avalia } \\
\text { a segurança da situação e encontra alguém para acompanhá-lo até o carro ou trazer } \\
\text { o carro para você/ (*você foi ao shopping sozinho) }\end{array}$ \\
\hline 2 & $\begin{array}{l}\text { liga para um familiar ou amigo para que o peguem/ caminha ao lado de outro (s) } \\
\text { freguês (s)/ chama um taxi }\end{array}$ \\
\hline $\mathbf{1}$ & $\begin{array}{l}\text { vai para o carro mas fica atento a algo suspeito/ caminha até o carro mas segura a } \\
\text { chave nas mãos como um artificio de proteção/ caminha até o carro mas mantém o } \\
\text { olhar no segurança/ caminha até o carro mas mantém o celular próximo }\end{array}$ \\
\hline $\mathbf{0}$ & "caminho até o carro" (sem reconhecimento que a situação possa ser insegura)/ "eu \\
\hline
\end{tabular}


não me preocupo com o ataque de outras pessoas"/ não sabe/ entra em pânico/ isso nunca aconteceria comigo/ "não estou preocupado"/ é uma má idéia ir para uma área como essa/ grita, chama a atenção e bate neles com alguma coisa

10. Você se encontra com dois amigos íntimos para um jantar em um restaurante que você está muito empolgado para conhecer. Entretanto, na chegada o gerente explica que um dos seus amigos não pode entrar porque ele/ela está vestido de forma muito casual. $\underline{\mathrm{O}}$ que você faria?

3 vai para outro lugar/ procura um outro restaurante, que permita traje informal e planeja visitar o restaurante novo outra hora/ janta no bar do restaurante em traje casual/ vê se seu amigo tem outras roupas com ele ou emprestado no restaurante (Q) ('seu amigo está vestindo jeans e tênis)

2 Resposta vaga indicando "você" "iria embora" sem especificar ir para outro restaurante ou quem está incluído (ex. "Eu deveria ir para o Mcdonalds")/ verificar o que seria necessário para ele ficar apropriadamente vestido (Q)/ reações extremas"fico muito bravo/vou embora e nunca mais volto"/ vaga resposta como "Eu discutiria isso com meu amigo e deixaria ele decidir"/ discutir com o gerente

1 fala para o seu amigo que está vestido casualmente ir para casa, mudar de roupa e voltar para o restaurante (você ficará esperando no bar do restaurante) ('seu amigo mora longe)/ procura uma loja próxima e compra algo apropriado/ tenta convencer ou subornar o gerente para deixar todos vocês entrarem/ quebra a regra/ reprograma tudo para uma outra noite

0 entra com o amigo vestido apropriadamente e deixa o amigo vestido informalmente para trás

11. Você está dirigindo na estrada na pista da esquerda quase na velocidade limite. Você repara que o carro atrás de você está correndo. $\underline{\text { O que você faria? }}$

3 dá sinal e muda de pista/ deixa o carro passar (Q)/ dá lugar em uma pista/ desvia o carro e o permite passar ou seguir em frente (com a implicação de mudar de pista mas não ficar inteiramente fora da estrada)/ resposta que conclui o objetivo de se afastar do motorista com pouco esforço, conseqüencia ou risco - e não envolver fisicamente sair da estrada ('é uma estrada de 4 pistas)

2 deixa eles passarem e liga 192 ou liga para pedir ajuda/ acelera e depois dá sinal para o carro passar/ (desacelerar ou acelerar com a insinuação de deixar o carro passar) e encosta no meio fio-desvia para a margem da estrada ou rampa de saida

1 respostas que envolvam se afastar da pista com exagero ou não envolvam deixar o carro passar ou é perigoso/ (ficar nervoso e) sai da estrada na próxima saída/ dirigir até a delegacia se parecer que o carro está seguindo você/ ligar 192/ sai da estrada no ponto disponível mais próximo/ "faço qualquer coisa para tentar se desviar deles"/ fazer sinal para eles passarem você $(\mathbf{Q})$ / vai devagar até que ele passe/ pisa 


\begin{tabular}{|l|l|}
\hline & $\begin{array}{l}\text { no freio momentaneamente para dar-lhes uma lição e depois deixa que ele passe/ } \\
\text { você não deveria dirigir na pista da esquerda (Q) }\end{array}$ \\
\hline 0 & $\begin{array}{l}\text { Faz nada/ continuo dirigindo na velocidade atual, não se preocupa/ continuo no } \\
\text { meu ritmo/ acelera/ pisa no freio com força/ pisa no freio levemente/ acelera e } \\
\text { depois desacelera/ coloca-os contra o carro próximo a você/ liga o "pisca-alerta" } \\
\text { (para fazer eles pensarem que você está pisando no freio" e depois desacelera/ abre } \\
\text { o vidro e acena para eles irem para trás/ somente continua andando até você achar } \\
\text { uma saída/ "deixo espaço suficiente então eles não irão bater em mim se eu tiver } \\
\text { que parar" }\end{array}$ \\
\hline
\end{tabular}

12. Você vê um cachorro pequeno e amigável, com uma coleira, sozinho na calçada na sua vizinhança. O que você faria?

\begin{tabular}{|l|l|}
\hline 3 & $\begin{array}{l}\text { tenta ter certeza se o cachorro é manso) - depois olha a informação na coleira e } \\
\text { procura ao redor pelo dono ou liga no número que está na identificação/ olha a } \\
\text { coleira e liga para a carrocinha ou abrigo de animais/ olha na coleira para } \\
\text { identificar o dono/ se você tem medo de cães, liga para centro humano ou liga para } \\
\text { ajuda }\end{array}$ \\
\hline 2 & $\begin{array}{l}\text { olha para a coleira (sem falar de ver identificação ou encontrar o proprietário)/ } \\
\text { resposta vaga sobre tentar localizar o dono ("tento identificar o cachorro e ligar } \\
\text { para o dono") (Q) sem mencionar olhar a coleira ou identificação/ liga para canil } \\
\text { municipal (Q) }\end{array}$ \\
\hline 1 & $\begin{array}{l}\text { uma resposta que envolva tentar fisicamente localizar o proprietário sem um plano } \\
\text { claro ou sem olhar para a coleira (ex. "pergunto para os clientes de lojas próximas } \\
\text { ou pessoas próximas na rua, se eles perderam um cachorro"/ pega o cachorro e bate } \\
\text { de porta em porta/ leva ele para casa e faz um anúncio/ pego ele e levo ao } \\
\text { veterinário/ }\end{array}$ \\
\hline $\mathbf{0}$ & $\begin{array}{l}\text { Nada, continuo andando/ deixa ele sozinho/ diz "oi" para o cachorro ou fala com o } \\
\text { cachorro e prossegue }\end{array}$ \\
\hline
\end{tabular}

13. Você está diagnosticado com uma rara doença de sangue que causa muito desconforto e deterioração cognitiva. Seu médico explica que um novo procedimento médico pode ajudar a curar a doença, mas esse procedimento tem uma taxa de mortalidade de $10 \%$. Alternativamente, uma medicação comumente usada pode proporcionar alívio de alguns sintomas. $\underline{O}$ que você faria?

3 pesquisa o assunto e cuidadosamente considera os riscos e beneficios - e então decide/ investiga sobre medicamentos alternativos/ faz muitas perguntas (Q)/ pega uma segunda opinião antes de decidir/ procura um alívio temporário e então uma segunda opinião 
\begin{tabular}{|l|l|}
\hline 2 & $\begin{array}{l}\text { escolhe um bom tratamento com boa base teórica/ escolhe um tratamento mas } \\
\text { reconhece que o outro possa ser uma opção viável/ tenta o remédio primeiro, e se } \\
\text { não funcionar procura a cura/ consideração sobre alternativas mas escolhe um sem } \\
\text { pesquisar mais/ segue o conselho do médico (e toma o remédio)/ "questiona sobre } \\
\text { os efeitos colaterais"(sem falar da necessidade de tomar uma decisão) }\end{array}$ \\
\hline $\mathbf{1}$ & $\begin{array}{l}\text { escolhe imediatamente uma opção sem uma análise racional (ex. "tento a } \\
\text { medicação" ou "faço a operação")/ respostas vagas "opto pelo tratamento mais } \\
\text { seguro" (Q)/ "vou atrás do auxílio que eu puder obter" (Q)/ "o que for menos caro } \\
\text { para os parentes"/ pega opinião de parentes/ procura o que fazer para ficar bem }\end{array}$ \\
\hline $\mathbf{0}$ & não sei/ faço nada \\
\hline
\end{tabular}

14. Você percebe que acidentalmente marcou dois compromissos na mesma tarde (em lados opostos da cidade). 0 que você faria?

\begin{tabular}{|l|l|}
\hline 3 & $\begin{array}{l}\text { liga para remarcar um deles (possivelmente aquele menos importante ou o mais } \\
\text { flexivel) e pede desculpas/ cancela um/ liga para explicar (* os compromissos são } \\
\text { aproximadamente } 60 \mathrm{Km} \text { de distância") }\end{array}$ \\
\hline 2 & $\begin{array}{l}\text { tenta cumprir os dois reduzindo um pouco de cada/ respostas vagas (ex. "ligo para } \\
\text { ambos, avalio as reações e depois decido")/ vai para o mais perto e depois vai para } \\
\text { o outro }\end{array}$ \\
\hline $\mathbf{1}$ & vai somente para um - ignora o outro \\
\hline $\mathbf{0}$ & $\begin{array}{l}\text { não vai para nenhum - pressão demais/ cancela ambos/ dou meus ingressos para } \\
\text { alguém (Q) }\end{array}$ \\
\hline
\end{tabular}

15. O gerente do seu banco avisa que os ganhos com a caderneta de poupança irão declinar significativamente nos próximos meses, enquanto que os ganhos com fundo de renda fixa irão aumentar. Atualmente, você só tem dinheiro na poupança e tem oportunidade de mudar seus investimentos após este aviso. $\underline{\text { O que você faria? }}$

\begin{tabular}{|l|l|}
\hline 3 & $\begin{array}{l}\text { transfere um pouco de dinheiro da poupança para renda fixa com objetivo de seguir } \\
\text { a recomendação mas evitar mudança tão drástica/ resposta que envolva mudar os } \\
\text { investimentos com poupança de alguma forma/ procura opinião de outros e depois } \\
\text { faz a mudança (* outros gerentes concordam) }\end{array}$ \\
\hline 2 & $\begin{array}{l}\text { respostas vagas "sigo a opinião porque eu confio nele" "mudo para renda fixa" } \\
\text { "tento ficar melhor financeiramente" (Q)/ procuro mais conselhos / pesquiso / não } \\
\text { sigo conselhos às cegas, uso meu próprio julgamento / espera até você ver } \\
\text { evidência de melhora nas aplicações de renda fixa antes de decidir }\end{array}$ \\
\hline $\mathbf{1}$ & $\begin{array}{l}\text { mudança drástica baseada nessa recomendação (ex. transferir todo o dinheiro da } \\
\text { poupança para renda fixa/ tirar o dinheiro da poupança mas não fazer mais } \\
\text { nenhuma aplicação bancária - investe todo o dinheiro em imóveis, ouro ou dólar/ } \\
\text { tirar todo o dinheiro da poupança e não guardar mais dinheiro em banco/ não muda } \\
\text { com análise racional (ex. faço nada já que você não pode me dizer o que vai }\end{array}$ \\
\hline
\end{tabular}


acontecer em seguida, "eu consultaria mais alguém mas no final ficaria com o dinheiro aplicado em poupança")

0 não sei/ não entendo nada sobre isso/ "nada"

ESCORE: O escore é a soma de todos os 15 itens $(\max =45)$

ESCORE TOTAL $=$

INFORMACÕES ADICIONAIS PARA ADMINISTRACÃO E PONTUAC̃̃o

- Quando necessário, repita a questão uma vez para garantir que o paciente entendeu.

- Anotar a resposta do paciente literalmente.

- Pontue enquanto conduz o teste e esteja SEGURO para questionar quando necessário: Questione quando houver $(Q)$ - com a instrução "Fale mais sobre isso" ou "Explique o que você quis dizer".

- Se, após ser questionado, o participante der informações adicionais que aumentem a pontuação da resposta, dê a ele/ela o valor mais alto.

Informações adicionais (em negrito) podem ser dadas se o participante perguntar diretamente sobre a informação ou der uma resposta que contradiga essa informação.

- Na questão 10, o gênero do amigo deve ser especificado como sendo o mesmo do participante.

- Se o participante der duas ou mais respostas , peça pra ele/ela escolher entre elas de forma direta: "Você disse X, Y e Z, - qual é a sua resposta final?"

- Se o participante dá muitas respostas mesmo depois de questionado, escolha a resposta que você acha que mais capta a resposta pretendida.

- Se o participante diz: "isso não se aplica a mim" (ex. "Eu não dirijo mais") peça para ele/ela imaginar que se aplica (ex. para esta questão, por favor imagine que você ainda dirige"). Se mesmo assim o participante mostrar-se relutante ou incapaz de conseguir esta perspectiva, dê a ele/ela resposta "0".

- Como regra geral, se o participante dá uma resposta com características de uma resposta de 3 pontos, mas de forma vaga, dê a ele 2 pontos. 


\section{ANEXO F - MODIFICAÇÃO 2}

Nome:

Data:

Idade:

Sexo:

\section{Teste de Julgamento Prático (TOP-J)}

INSTRUCÕES: Agora eu gostaria que você me falasse algumas soluções para problemas ou situações cotidianos. Por favor, seja o mais claro possivel em suas respostas.

1. Você está de férias longe de casa e percebe que não tem comprimidos controlados suficientes para toda a viagem. Você não pode ficar sem eles e nem voltar para casa. O que você faria?

\begin{tabular}{|l|l|}
\hline 3 & $\begin{array}{l}\text { procura um médico (ou plano de saúde) e pega uma receita por telefone ou fax em } \\
\text { uma farmácia local/ liga para casa e pede para alguém mandar os remédios Sedex/ } \\
\text { liga para um médico ou farmácia local (Q) (e consegue uma receita)/ liga para a } \\
\text { farmácia onde você costuma comprar e pergunta se eles tem o histórico das suas } \\
\text { receitas no computador }\end{array}$ \\
\hline $\mathbf{2}$ & $\begin{array}{l}\text { vai ao médico local/Pronto Socorro/hospital local e pede uma receita ou descreve o } \\
\text { problema/ leva os remédios a uma farmácia local e vê se eles conseguem ajudar/ } \\
\text { (*você não tem a receita com você)/ pega amostra grátis ou receita em um médico } \\
\text { ou farmácia/ convence o farmacêutico a te dar pillulas suficientes }\end{array}$ \\
\hline $\mathbf{1}$ & $\begin{array}{l}\text { toma doses menores para durarem mais/ economiza-os (raciona-os)/ respostas } \\
\text { vagas mesmo após questionado (ex. "vou à farmácia e pego", "ligo para casa e } \\
\text { peço ajuda", "consigo que o farmacêutico envie por e-mail", "consigo algum") }\end{array}$ \\
\hline $\mathbf{0}$ & $\begin{array}{l}\text { espera para ver se você precisa deles, não há motivo para se preocupar adiantado/ } \\
\text { nada, espero até chegar em casa/ espera até alguma coisa ruim acontecer/ não sei, } \\
\text { continuo sem/ evito esforço físico }\end{array}$ \\
\hline
\end{tabular}

2. Você recebe um telefonema avisando que acabou de ganhar um carro em um sorteio do qual participou muitos meses antes. A pessoa que ligou pede pára você fornecer seu endereço, CPF e número do cartão de crédito. 0 que você faria?

3 não fornece informações e pede para enviarem um documento por escrito declarando termos e condições do sorteio/ pede uma comprovação da autenticidade por escrito antes de fornecer informações/ fornece o endereço para que possam enviar-lhe comprovação ou checa com o Serviço de Proteção ao Consumidor

2 desliga/ não fornece informação alguma (mas não solicita informações adicionais/ esclarecimento ou vaga referência sobre reunir mais informações - ex., "eles necessitam provar isso para mim")/ não interessado/ não fornece informações por telefone/ tento pegar identificação deles $(\mathbf{Q}) /$ pede o número deles e liga de volta 


\begin{tabular}{|l|l|}
\hline & $\begin{array}{l}\text { para conferir (*o patrocinador do sorteio não é local)/ não dá informações mas } \\
\text { faz perguntas/ avisa a policia (Q) }\end{array}$ \\
\hline 1 & $\begin{array}{l}\text { pede mais informações por telefone e depois fornece as informações solicitadas } \\
\text { (alguma comprovação é pedida, mas não por escrito)/ somente fornece o endereço } \\
(\mathbf{Q})\end{array}$ \\
\hline $\mathbf{0}$ & $\begin{array}{l}\text { fornece informações (não solicita informações ou esclarecimentos posteriores de } \\
\text { forma alguma)/ somente fornece endereço e CPF }\end{array}$ \\
\hline
\end{tabular}

3. Você está fora da cidade visitando parentes e de repente lembra que pode ter deixado o fogão da sua casa ligado. 0 que você faria?

\begin{tabular}{|l|l|}
\hline 3 & $\begin{array}{l}\text { entra em contato com alguém que possa ir na sua casa checar (ex. vizinho, } \\
\text { proprietário, membro da família) }\end{array}$ \\
\hline $\mathbf{2}$ & liga para a polícia ou bombeiro \\
\hline $\mathbf{1}$ & pânico/ corre para casa (*você está longe de casa) \\
\hline $\mathbf{0}$ & $\mathrm{faz}$ nada/ não sabe \\
\hline
\end{tabular}

4. Você leu uma notícia de que o governo vai reduzir mensalmente os pagamentos do INSS de $R \$ 1500,00$ para $R \$ 750,00$ para uma determinada porcentagem de pensionistas. $\underline{O}$ que você faria?

3 tenta descobrir a probabilidade dos seus beneficios serem reduzidos/liga para obter mais informações na tentativa de determinar se isso afeta você/ ligar para o INSS para saber mais $(\mathbf{Q})$ - se você será afetado

2 Tenta vagamente obter mais informações sem tentar determinar diretamente se você será afetado ou supor que será afetado (ex. "eu verifico, porque isso não está certo" "determino a validade da informação" "ligo e vejo o que posso fazer (Q)"/ liga para o senador para obter informação $(\mathbf{Q}) /$ pesquisa o problema ou "procura o motivo" (sem averiguar se os beneficios irão mudar)/ pesquisa quanto de redução haverá/ procuro saber se vou ser afetado e se eu for, entro na justiça

1 reduz os gastos mensais/ deixa as contas pagas para manter o orçamento controlado/ vai trabalhar/ pede dinheiro emprestado/ ajusta as finanças/ entro na justiça

0 faz nada/ espera para ver o que vai acontecer/ isso não me afeta/ não se pode brigar com o governo $(Q) /$ diz para o governo que isso é uma má idéia/ reclama ou liga para jornais locais/ não acredito nisso/ eu só vivo com meus recursos/ escrevo para o meu senador/deputado e me queixo/ fico bravo

5. Você está em um jantar na casa de um amigo, mas o prato principal está com gosto de estragado. $\underline{\mathbf{Q} q u e \text { você faria? }}$ 


\begin{tabular}{|l|l|}
\hline 3 & $\begin{array}{l}\text { para de comer e avisa o anfitrião discretamente/ avalia a reação das pessoas e diz } \\
\text { alguma coisa para o anfitrião (querendo dizer que é melhor parar de comer ou que } \\
\text { algo está errado) }\end{array}$ \\
\hline 2 & $\begin{array}{l}\text { para de comer (mas sem falar para o anfitrião ou outros convidados)/ come os } \\
\text { acompanhamentos/ separa a parte ruim/ fala para o anfitrião e para os outros "isto } \\
\text { está com o gosto ruim para mim, está bom para vocês?" (Q)/ pede para o anfitrião } \\
\text { provar/ fala alto para os outros convidados (sem falar primeiro para o anfitrião) }\end{array}$ \\
\hline $\mathbf{1}$ & $\begin{array}{l}\text { respostas vagas como empurrar a comida ao redor do prato/ outras respostas vagas } \\
(\mathbf{Q}) / \text { pergunta sobre os ingredientes/ mente para os outros (ex. "Eu sou alérgico") } \\
\text { ou propositalmente desvia a atenção da comida }\end{array}$ \\
\hline $\mathbf{0}$ & $\begin{array}{l}\text { continua comendo/ faz nada - não queria ofender um amigo/ se vira com o que for } \\
\text { possivel (Q)/ somente come um pouco }\end{array}$ \\
\hline
\end{tabular}

6. Você toma uma medicação diária para reduzir o colesterol, mas um estudo novo publicado no jornal local sugere que esta medicação está relacionada a um alto risco para câncer. $\underline{O \text { que você faria? }}$

\begin{tabular}{|l|l|}
\hline 3 & $\begin{array}{l}\text { procura saber mais sobre o estudo (ex. como a pesquisa foi conduzida, se outros } \\
\text { dados apóiam os achados, que tipo de câncer)/ liga para um especialista/ liga para } \\
\text { seu médico/ pergunta sobre medicamentos alternativos }\end{array}$ \\
\hline $\mathbf{2}$ & $\begin{array}{l}\text { tenta passivamente discernir a validade do estudo (ex. "espero por mais } \\
\text { confirmações"/ "ignoro isso até que seja verificado por outro estudo") }\end{array}$ \\
\hline $\mathbf{1}$ & $\begin{array}{l}\text { para de tomar medicação e depois obtém maiores informações do médico ou sites } \\
\text { médicos/ para de tomar a medicação/ fala para o médico e insiste em parar de } \\
\text { tomar a medicação/ continua tomando a medicação (com boa justificativa) }\end{array}$ \\
\hline $\mathbf{0}$ & $\begin{array}{l}\text { ignora os achados/ faz nada/ ri da publicação - isso é impossível/ continua } \\
\text { tomando a medicação (com justificativa ruim - ex. "Eu sou velho, quem se importa } \\
\text { se eu tiver câncer") }\end{array}$ \\
\hline
\end{tabular}

7. Você está começando a ter problemas para dirigir devido a problemas na visão noturna e discreta confusão mental. O que você faria?

3 Faz uma consulta com um profissional, como um médico ou avaliação de direção para determinar a causa do problema (e para de dirigir a noite até que a causa seja determinada/ enquanto espera a opinião do médico)

2 evita dirigir a noite/ somente dirige para lugares que você conhece bem/ ajusta os modelos de direção $(\mathbf{Q})$ / pede para esposa ou amigo dirigir a noite/ vaga referência sobre saber mais sobre o problema como "ir ao médico" $(\mathbf{Q})$ ou "peço para minha esposa me supervisionar e deixo ela dirigir mais"

1 desiste completamente de dirigir/ deixar de dirigir (sem menção de estar sendo 
avaliado)/ discutir com a família e desistir de dirigir/ "Eu não dirijo" (Q)/ ter alguém para dirigir para mim/ "segurança vem primeiro, mas eu não tenho certeza"

0 faz nada/ "não saio de casa a noite" (Q)/ saio somente se não estiver chovendo

8. Você acha uma carteira na calçada. Você a pega e 5 passos depois você acha uma nota de $R \$ 50,00$. O que você faria?

3 qualquer resposta que busque determinar quem é o dono ou devolver ambos a carteira e o dinheiro e reconhecer a possibilidade de que não pertençam à mesma pessoa/ levar a carteira à polícia ou prefeitura e explicar sobre a nota/ procurar o RG na carteira e ligar para a pessoa para verificar a quantia na carteira (e devolver ambos)

2 qualquer resposta que busque tentar determinar o dono/ devolver a carteira mas não a nota que possivelmente não estava na carteira (ex. procurar o RG na carteira e tentar encontrar o dono)/ levá-la à polícia/ qualquer resposta dizendo que a carteira e a nota deveriam ser devolvidas a um único dono/ leva a carteira ou ambos à polícia

1 leva a carteira para a delegacia mas fica com a nota/ "devolvo a carteira, e se eu gostar da pessoa devolvo a nota, do contrário fico com ela"/ fica com a nota mas tenta devolver a carteira

0 fica com ambos/ somente continua andando/ "eu pegaria e sairia correndo achando que é uma armadilha"

9. Você foi dirigindo a um shopping em uma cidade vizinha, e quando acabou de fazer as compras estava escuro lá fora. Seu carro está parado a uma grande distância da saída e lá tem acontecido muitos assaltos recentemente. $\underline{\mathbf{O} \text { que você }}$ faria?

3 acha um segurança ou policial e pede a eles para acompanhá-lo ao seu carro/ avalia a segurança da situação e encontra alguém para acompanhá-lo até o carro ou trazer o carro para você/ (*você foi ao shopping sozinho)

2 liga para um familiar ou amigo para que o peguem/ caminha ao lado de outro (s) freguês (s)/ chama um taxi

1 vai para o carro mas fica atento a algo suspeito/ caminha até o carro mas segura a chave nas mãos como um artificio de proteção/ caminha até o carro mas mantém o olhar no segurança/ caminha até o carro mas mantém o celular próximo

0 "caminho até o carro" (sem reconhecimento que a situação possa ser insegura)/ "eu não me preocupo com o ataque de outras pessoas"/ não sabe/ entra em pânico/ isso nunca aconteceria comigo/ "não estou preocupado"/ é uma má idéia ir para uma área como essa/ grita, chama a atenção e bate neles com alguma coisa 
10. Você se encontra com dois amigos íntimos para um jantar em um restaurante que você está muito empolgado para conhecer. Entretanto, na chegada o gerente explica que um dos seus amigos não pode entrar porque ele/ela está vestido de forma muito casual. $\underline{O}$ que você faria?

\begin{tabular}{|l|l|}
\hline 3 & $\begin{array}{l}\text { vai para outro lugar/ procura um outro restaurante, que permita traje informal e } \\
\text { planeja visitar o restaurante novo outra hora/ janta no bar do restaurante em traje } \\
\text { casual/ vê se seu amigo tem outras roupas com ele ou emprestado no restaurante } \\
\text { (Q) ("seu amigo está vestindo jeans e tênis) }\end{array}$ \\
\hline $\mathbf{2}$ & $\begin{array}{l}\text { Resposta vaga indicando "você" "iria embora" sem especificar ir para outro } \\
\text { restaurante ou quem está incluído (ex. "Eu deveria ir para o Mcdonalds")/ verificar } \\
\text { o que seria necessário para ele ficar apropriadamente vestido (Q)/ reações } \\
\text { extremas"fico muito bravo/vou embora e nunca mais volto"/ vaga resposta como } \\
\text { "Eu discutiria isso com meu amigo e deixaria ele decidir"/ discutir com o gerente }\end{array}$ \\
\hline $\mathbf{1}$ & $\begin{array}{l}\text { fala para o seu amigo que está vestido casualmente ir para casa, mudar de roupa e } \\
\text { voltar para o restaurante (você ficará esperando no bar do restaurante) ("seu amigo } \\
\text { mora longe)/ procura uma loja próxima e compra algo apropriado/ tenta convencer } \\
\text { ou subornar o gerente para deixar todos vocês entrarem/ quebra a regra/ } \\
\text { reprograma tudo para uma outra noite }\end{array}$ \\
\hline $\mathbf{0}$ & $\begin{array}{l}\text { entra com o amigo vestido apropriadamente e deixa o amigo vestido informalmente } \\
\text { para trás }\end{array}$ \\
\hline
\end{tabular}

11. Você está dirigindo na estrada na pista da esquerda quase na velocidade limite. Você repara que o carro atrás de você está correndo. O que você faria?

\begin{tabular}{|l|l|}
\hline 3 & $\begin{array}{l}\text { dá sinal e muda de pista/ deixa o carro passar (Q)/ dá lugar em uma pista/ desvia o } \\
\text { carro e o permite passar ou seguir em frente (com a implicação de mudar de pista } \\
\text { mas não ficar inteiramente fora da estrada)/ resposta que conclui o objetivo de se } \\
\text { afastar do motorista com pouco esforço, conseqüência ou risco - e não envolver } \\
\text { fisicamente sair da estrada ("é uma estrada de 4 pistas) }\end{array}$ \\
\hline 2 & $\begin{array}{l}\text { deixa eles passarem e liga 192 ou liga para pedir ajuda/ acelera e depois dá sinal } \\
\text { para o carro passar/ (desacelerar ou acelerar com a insinuação de deixar o carro } \\
\text { passar) e encosta no meio fio-desvia para a margem da estrada ou rampa de saída }\end{array}$ \\
\hline 1 & $\begin{array}{l}\text { respostas que envolvam se afastar da pista com exagero ou não envolvam deixar o } \\
\text { carro passar ou é perigoso/ (ficar nervoso e) sai da estrada na próxima saída/ dirigir } \\
\text { até a delegacia se parecer que o carro está seguindo você/ ligar 192/ sai da estrada } \\
\text { no ponto disponivel mais próximo/ "faço qualquer coisa para tentar se desviar } \\
\text { deles"/ fazer sinal para eles passarem você (Q)/ vai devagar até que ele passe/ pisa } \\
\text { no freio momentaneamente para dar-lhes uma lição e depois deixa que ele passe/ } \\
\text { você não deveria dirigir na pista da esquerda (Q) }\end{array}$ \\
\hline
\end{tabular}


\begin{tabular}{|l|l|}
\hline & Faz nada/ continuo dirigindo na velocidade atual, não se preocupa/ continuo no \\
meu ritmo/ acelera/ pisa no freio com força/ pisa no freio levemente/ acelera e \\
depois desacelera/ coloca-os contra o carro próximo a você/ liga o "pisca-alerta" \\
(para fazer eles pensarem que você está pisando no freio" e depois desacelera/ abre \\
o vidro e acena para eles irem para trás/somente continua andando até você achar \\
uma saída/ "deixo espaço suficiente então eles não irão bater em mim se eu tiver \\
que parar"
\end{tabular}

12. Você vê um cachorro pequeno e amigável, com uma coleira, sozinho na calçada na sua vizinhança. $\underline{O}$ que você faria?

\begin{tabular}{|l|l|}
\hline 3 & $\begin{array}{l}\text { tenta ter certeza se o cachorro é manso) - depois olha a informação na coleira e } \\
\text { procura ao redor pelo dono ou liga no número que está na identificação/ olha a } \\
\text { coleira e liga para a carrocinha ou abrigo de animais/ olha na coleira para } \\
\text { identificar o dono/ se você tem medo de cães, liga para centro humano ou liga para } \\
\text { ajuda }\end{array}$ \\
\hline 2 & $\begin{array}{l}\text { olha para a coleira (sem falar de ver identificação ou encontrar o proprietário)/ } \\
\text { resposta vaga sobre tentar localizar o dono ("tento identificar o cachorro e ligar } \\
\text { para o dono") (Q) sem mencionar olhar a coleira ou identificação/ liga para canil } \\
\text { municipal (Q) }\end{array}$ \\
\hline 1 & $\begin{array}{l}\text { uma resposta que envolva tentar fisicamente localizar o proprietário sem um plano } \\
\text { claro ou sem olhar para a coleira (ex. "pergunto para os clientes de lojas próximas } \\
\text { ou pessoas próximas na rua, se eles perderam um cachorro"/ pega o cachorro e bate } \\
\text { de porta em porta/ leva ele para casa e faz um anúncio/ pego ele e levo ao } \\
\text { veterinário/ }\end{array}$ \\
\hline 0 & $\begin{array}{l}\text { Nada, continuo andando/ deixa ele sozinho/ diz "oi" para o cachorro ou fala com o } \\
\text { cachorro e prossegue }\end{array}$ \\
\hline
\end{tabular}

13. Você está diagnosticado com uma rara doença de sangue que causa muito desconforto e problemas no cérebro. Seu médico explica que um novo procedimento médico pode ajudar a curar a doença, mas esse procedimento tem uma taxa de mortalidade de $10 \%$. Alternativamente, uma medicação comumente usada pode proporcionar alívio de alguns sintomas. O que você faria?

3 pesquisa o assunto e cuidadosamente considera os riscos e benefícios - e então decide/ investiga sobre medicamentos alternativos/ faz muitas perguntas (Q)/ pega uma segunda opinião antes de decidir/ procura um alívio temporário e então uma segunda opinião

2 escolhe um bom tratamento com boa base teórica/ escolhe um tratamento mas reconhece que o outro possa ser uma opção viável/ tenta o remédio primeiro, e se não funcionar procura a cura/ consideração sobre alternativas mas escolhe um sem pesquisar mais/ segue o conselho do médico (e toma o remédio)/ "questiona sobre os efeitos colaterais"(sem falar da necessidade de tomar uma decisão) 


\begin{tabular}{|l|l|}
\hline 1 & $\begin{array}{l}\text { escolhe imediatamente uma opção sem uma análise racional (ex. "tento a } \\
\text { medicação" ou "faço a operação")/ respostas vagas "opto pelo tratamento mais } \\
\text { seguro" (Q)/ "vou atrás do auxílio que eu puder obter" (Q)/ "o que for menos caro } \\
\text { para os parentes"/ pega opinião de parentes/ procura o que fazer para ficar bem }\end{array}$ \\
\hline $\mathbf{0}$ & não sei/ faço nada \\
\hline
\end{tabular}

14. Você percebe que acidentalmente marcou dois compromissos na mesma tarde (em lados opostos da cidade). O que você faria?

3 liga para remarcar um deles (possivelmente aquele menos importante ou o mais flexível) e pede desculpas/ cancela um/ liga para explicar (* os compromissos são aproximadamente $60 \mathrm{Km}$ de distância")

2 tenta cumprir os dois reduzindo um pouco de cada/ respostas vagas (ex. "ligo para ambos, avalio as reações e depois decido")/ vai para o mais perto e depois vai para o outro

1 vai somente para um - ignora o outro

0 não vai para nenhum - pressão demais/ cancela ambos/ dou meus ingressos para alguém (Q)

15. O gerente do seu banco avisa que os ganhos com a caderneta de poupança irão declinar significativamente nos próximos meses, enquanto que os ganhos com outra aplicação que tem risco irão aumentar. Atualmente, você só tem dinheiro na poupança e tem oportunidade de mudar seus investimentos após este aviso. $\underline{O \text { que }}$ você faria?

\begin{tabular}{|l|l|}
\hline 3 & $\begin{array}{l}\text { transfere um pouco de dinheiro da poupança para renda fixa com objetivo de seguir } \\
\text { a recomendação mas evitar mudança tão drástica/ resposta que envolva mudar os } \\
\text { investimentos com poupança de alguma forma/ procura opinião de outros e depois } \\
\text { faz a mudança (* outros gerentes concordam) }\end{array}$ \\
\hline 2 & $\begin{array}{l}\text { respostas vagas "sigo a opinão porque eu confio nele" "mudo para renda fixa" } \\
\text { "tento ficar melhor financeiramente" (Q)/ procuro mais conselhos / pesquiso / não } \\
\text { sigo conselhos às cegas, uso meu próprio julgamento / espera até você ver } \\
\text { evidência de melhora nas aplicações de renda fixa antes de decidir }\end{array}$ \\
\hline 1 & $\begin{array}{l}\text { mudança drástica baseada nessa recomendação (ex. transferir todo o dinheiro da } \\
\text { poupança para renda fixa/ tirar o dinheiro da poupança mas não fazer mais } \\
\text { nenhuma aplicação bancária - investe todo o dinheiro em imóveis, ouro ou dólar/ } \\
\text { tirar todo o dinheiro da poupança e não guardar mais dinheiro em banco/ não muda } \\
\text { com análise racional (ex. faço nada já que você não pode me dizer o que vai } \\
\text { acontecer em seguida, "eu consultaria mais alguém mas no final ficaria com o } \\
\text { dinheiro aplicado em poupança") }\end{array}$ \\
\hline $\mathbf{0}$ & não sei/ não entendo nada sobre isso/ "nada" \\
\hline
\end{tabular}


ESCORE: O escore é a soma de todos os 15 itens ( $\max =45)$

ESCORE TOTAL $=$

\section{INFORMACÕES ADICIONAIS PARA ADMINISTRACÃO E PONTUACÃO}

- Quando necessário, repita a questão uma vez para garantir que o paciente entendeu.

- Anotar a resposta do paciente literalmente.

- Pontue enquanto conduz o teste e esteja SEGURO para questionar quando necessário: Questione quando houver $(Q)$ - com a instrução "Fale mais sobre isso" ou "Explique o que você quis dizer".

- Se, após ser questionado, o participante der informações adicionais que aumentem a pontuação da resposta, dê a ele/ela o valor mais alto.

Informações adicionais (em negrito) podem ser dadas se o participante perguntar diretamente sobre a informação ou der uma resposta que contradiga essa informação.

- Na questão 10, o gênero do amigo deve ser especificado como sendo o mesmo do participante.

- Se o participante der duas ou mais respostas, peça pra ele/ela escolher entre elas de forma direta: "Você disse $\mathrm{X}, \mathrm{Y}$ e Z, - qual é a sua resposta final?"

- Se o participante dá muitas respostas mesmo depois de questionado, escolha a resposta que você acha que mais capta a resposta pretendida.

- Se o participante diz: "isso não se aplica a mim" (ex. "Eu não dirijo mais") peça para ele/ela imaginar que se aplica (ex. para esta questão, por favor imagine que você ainda dirige"). Se mesmo assim o participante mostrar-sè relutante ou incapaz de conseguir esta perspectiva, dê a ele/ela resposta " 0 ".

- Como regra geral, se o participante dá uma resposta com características de uma resposta de 3 pontos, mas de forma vaga, dê a ele 2 pontos. 


\section{ANEXO G - RETROTRADUÇÃO}

Name:

Date:

Age:

Sex:

\section{Test of Practical Judgment (TOP-J)}

INSTRUCTIONS: Now I'd like you to tell me some solutions to daily problems or situations. Please, be clearest as possible in your answers.

1. You are on vacation far from home and notice that you don't have enough controlled pills for the entire trip. You can stay neither without your pills nor come back home. What would you do?

\begin{tabular}{|l|l|}
\hline 3 & $\begin{array}{l}\text { look for a doctor (or health plan) and gets a prescription by phone or fax in a local } \\
\text { drugstore/ calls home and asks for somebody to order his/her medications by } \\
\text { Sedex/ calls a local doctor or drugstore (Q) (and gets a prescription)/ calls a } \\
\text { drugstore where uses to shop and asks if they have his/her prescriptions report in } \\
\text { their computer }\end{array}$ \\
\hline 2 & $\begin{array}{l}\text { goes to a local doctor/emergency/hospital and asks for a prescription or describes } \\
\text { the problem/ takes his/her medications to a local drugstore and sees if they can help } \\
\text { (*you don't have the prescription with you)/ gets free samples or a prescription in } \\
\text { a doctor or drugstore/talks the druggist into to give enough pills to him/her }\end{array}$ \\
\hline $\mathbf{1}$ & $\begin{array}{l}\text { takes smaller doses for it to last longer/ saves them (spares them)/ vague answers } \\
\text { even after questioned (e.g., "I go to the drugstore and get it", "I call home and ask } \\
\text { for help", "I get the druggist to send me by e-mail", "I get some") }\end{array}$ \\
\hline $\mathbf{0}$ & $\begin{array}{l}\text { waits to see if needs them, there's no reason to be worried in advance / does } \\
\text { nothing, I wait until coming home/ waits until some bad thing happens/ I don't } \\
\text { know, I keep without them/I avoid physical effort }\end{array}$ \\
\hline
\end{tabular}

2. You receive a phone call saying that you just got a car in a prize draw which you took part many months before. The person who calls asks your address, ID, and credit card number. What would you do?

3 gives no information and asks him to send a written document declaring terms and conditions of the drawing/ asks for an written evidence of the authenticity before giving the information/ gives his/her address so that they can send proof to it or 


\begin{tabular}{|l|l|}
\hline & check with the Consumer Protection Service (Product Safety Commission) \\
\hline 2 & $\begin{array}{l}\text { rings off/ gives no information (but doesn't ask for additional information/ } \\
\text { explanations or vague reference on gathering more information - e.g., "they need } \\
\text { to prove this for me")/ not interested/ doesn't give information by telephone/ I try } \\
\text { to get their identification }(\mathbf{Q}) / \text { asks for their number and call in return to check (* } \\
\text { the drawing sponsor is not local)/gives no information but makes questions/ calls } \\
\text { the police (Q) }\end{array}$ \\
\hline $\mathbf{1}$ & $\begin{array}{l}\text { asks for more information by phone and then gives the requested information } \\
\text { (some proof is asked for, but not in writing)/ gives only the address (Q) }\end{array}$ \\
\hline $\mathbf{0}$ & $\begin{array}{l}\text { gives information (doesn't ask for information or additional explanations anyway)/ } \\
\text { just gives address and ID }\end{array}$ \\
\hline
\end{tabular}

3. You are out of the city visiting relatives and suddenly you remember that you may have left the stove in your house on. What would you do?

\begin{tabular}{|l|l|}
\hline 3 & $\begin{array}{l}\text { makes contact with someone who can go in his/her house to check (e.g., neighbor, } \\
\text { owner, member of the family) }\end{array}$ \\
\hline $\mathbf{2}$ & calls the police or the fire department \\
\hline $\mathbf{1}$ & Panic/ runs for home ('you are far from home) \\
\hline $\mathbf{0}$ & makes nothing/ doesn't know \\
\hline
\end{tabular}

4. You read a newspaper matter about government reducing monthly contributions to Social Security from $R \$ 1500,00$ to $R \$ 750,00$ for a certain number of pensioners. What would you do?

\begin{tabular}{|l|l|}
\hline 3 & $\begin{array}{l}\text { tries to find the probability of his/her benefits being reduced/ calls to get more } \\
\text { information in order to find if this concerns to him/ calls to Social Security to know } \\
\text { more (Q) - if you will be affected }\end{array}$ \\
\hline 2 & $\begin{array}{l}\text { tries vaguely to get more information without trying to find directly if you will be } \\
\text { affected or to suppose that will be affected (e.g." II check because this is not right" } \\
\text { "check the validity of the information" "call and see what I can do (Q)"/ calls to } \\
\text { the senator to get information (Q)/ analyses the matter or "looks for the reason" } \\
\text { (without checking if the benefits will change)/ try to find how much reduction there } \\
\text { will be/ I try to know if I will be affected and if so, I will settle a lawsuit }\end{array}$ \\
\hline $\mathbf{1}$ & $\begin{array}{l}\text { Curtails expenses/ pay all bills to keep budget under control/ goes to work/ asks for } \\
\text { loaned money/ fit finances/ I settle a lawsuit }\end{array}$ \\
\hline $\mathbf{0}$ & $\begin{array}{l}\text { does nothing/ waits to see what happens/ that doesn't concerns me/ we can't fight } \\
\text { the government (Q)/ says for the government that this is an bad idea/ complains or } \\
\text { calls for local newspaper/ I do not believe this/ I only live with my resources/ I } \\
\text { write for my senator/congressman and complain/I get mad }\end{array}$ \\
\hline
\end{tabular}


5. You're dinning at your friend's house but the main dish tastes bad. What would you do?

\begin{tabular}{|l|l|}
\hline 3 & $\begin{array}{l}\text { stops eating and carefully says it to his/her host/ checks other people reaction and } \\
\text { says something to his/her host (wanting to say that it is better to stop eating or that } \\
\text { something's wrong) }\end{array}$ \\
\hline 2 & $\begin{array}{l}\text { stops eating (but without saying to his/her host or other guests)/ eats side dishes/ } \\
\text { puts aside bad food/ says for the host and for the others "this is with a bad taste for } \\
\text { me, is it good for you?" (Q)/ asks host to prove it/ speaks loud to other guests } \\
\text { (without speaking first to host) }\end{array}$ \\
\hline $\mathbf{1}$ & $\begin{array}{l}\text { vague answers like to put aside food in dish/ other vague answers (Q)/ asks for } \\
\text { ingredients/ lies to others (e.g., "I'm allergic") or deviates attention from food } \\
\text { deliberately }\end{array}$ \\
\hline $\mathbf{0}$ & $\begin{array}{l}\text { continues eating/ does nothing - didn't want to hurt a friend/ gets by with what's } \\
\text { possible (Q)/ eats only a few }\end{array}$ \\
\hline
\end{tabular}

6. You take cholesterol pills daily but a recent published study in the local newspaper suggests that this drug is associated to a high risk for cancer. What would you do?

\begin{tabular}{|l|l|}
\hline 3 & $\begin{array}{l}\text { Tries to know more about the study (e.g., how research was made, if other data } \\
\text { support the findings, what type of cancer)/ calls to a expert/ calls to his/her doctor/ } \\
\text { asks for alternative medicines }\end{array}$ \\
\hline $\mathbf{2}$ & $\begin{array}{l}\text { tries passively to find the validity of the study (e.g., "I wait for more } \\
\text { confirmations"/"I ignore this until it is corroborated another study") }\end{array}$ \\
\hline $\mathbf{1}$ & $\begin{array}{l}\text { stops taking his/her medicine and later gets more information from doctor or } \\
\text { medical sites/ stops taking medicine/ talks with a doctor and insists on stopping } \\
\text { taking his/her medicine/ continues taking his/her medicine (with good reasoning) }\end{array}$ \\
\hline $\mathbf{0}$ & $\begin{array}{l}\text { ignores the findings/ does nothing/ laughs at the publication - this is impossible/ } \\
\text { continues taking his/her medicine (with bad reasoning - e.g., "I'm old, who will } \\
\text { matter if I have cancer") }\end{array}$ \\
\hline
\end{tabular}

7. You are starting to have problems to driving due to problems with nocturnal vision and mild mental confusion. What would you do?

3 makes an appointment with a professional, as a doctor or driving exam to find the cause of the problem (and stops to drive at night until the cause is found while waits doctor's opinion) 
\begin{tabular}{|l|l|}
2 & $\begin{array}{l}\text { Avoids driving at night/ drives only direct to places he/she knows well/ adjusts the } \\
\text { direction models (Q)/ asks his wife or friend to drive at night/ vague mention about } \\
\text { trying to know more about the problem as "go to doctor" (Q) or "I ask my wife to } \\
\text { supervise me and let her drive more" }\end{array}$ \\
\hline 1 & $\begin{array}{l}\text { gives up driving completely/ stop driving (without mention to be being evaluated)/ } \\
\text { discuss with family and give up driving "I do not drive" (Q)/ have somebody to } \\
\text { drive for me "security comes first, but I am not certain" }\end{array}$ \\
\hline $\mathbf{0}$ & Does nothing "I do not leave home at night" (Q)/I only go out if it's not raining \\
\hline
\end{tabular}

8. You find a wallet in the sidewalk. You take it and 5 steps later you find an R\$ 50,00 bill. What would you do?

\begin{tabular}{|l|l|}
\hline 3 & $\begin{array}{l}\text { any answer that tries to find who is the owner or to give back both wallet and } \\
\text { money and to recognize the possibility that they do not belong to the same person/ } \\
\text { to take the wallet to police or city hall and to explain about the bill/ to look for ID } \\
\text { in the wallet and to call for the person to verify the amount of money in the wallet } \\
\text { (and to return both) }\end{array}$ \\
\hline $\mathbf{2}$ & $\begin{array}{l}\text { any answer that tries to find the owner/ to give back the wallet but not the bill } \\
\text { which possibly wasn't in the wallet (e.g., to look for the ID in the wallet and to try } \\
\text { to find the owner)/ to take it the police/ any answer stating that the wallet and the } \\
\text { bill should be gave back to a sole owner/ takes the wallet or both to the police }\end{array}$ \\
\hline $\mathbf{1}$ & $\begin{array}{l}\text { takes the wallet for the police station but stays with the bill/ "I give back the wallet, } \\
\text { and if I like the guy, I give back the note, on the contrary, I stay with it"/ stays with } \\
\text { the bill but tries to give back the wallet }\end{array}$ \\
\hline $\mathbf{0}$ & $\begin{array}{l}\text { stays with both/ just keeps walking/ "I would catch and leave running - thinking } \\
\text { that it is a trap" }\end{array}$ \\
\hline
\end{tabular}

9. You went driving to a shopping mall in a neighbor city, and as you finished shopping it was dark outside. Your car is parked at a great distance from exit and there has been much robbery over there recently. What would you do?

\begin{tabular}{|l|l|}
\hline 3 & $\begin{array}{l}\text { finds a security guard or policeman and asks them to following him/her to his/her } \\
\text { ca/r evaluates the security of the situation and finds somebody to follow him/her to } \\
\text { the car or to bring the car for you ("you went to the mall alone) }\end{array}$ \\
\hline $\mathbf{2}$ & $\begin{array}{l}\text { Calls a relative or friend so that they can get it/ walks by the side of another(s) } \\
\text { customer(s)/ calls a taxi }\end{array}$ \\
\hline $\mathbf{1}$ & $\begin{array}{l}\text { goes to the car but stays careful from something suspicious/ walks to the car but } \\
\text { keeps the keys in hands as a protection artifice/ walks to the car but keeps looking } \\
\text { to the security guard/ walks to the car but keeps cell phone close }\end{array}$ \\
\hline $\mathbf{0}$ & $\begin{array}{l}\text { "walk to the car" (without recognition that the situation can be unsafe)/“I am not } \\
\text { worried about the attack of other people"/I do not know/ I panic/ this would never } \\
\text { happen to me/"I am not worried"/ it's a bad idea to go to a place like this/ cries out, } \\
\text { calls attention and beats them with something }\end{array}$ \\
\hline
\end{tabular}


10. You meet with two close friends to dinner in a restaurant that you are very excited to know. However, in the arrival the manager explains that one of your friends cannot get in because he/she is dressed very casual. What would you do?

\begin{tabular}{|l|l|}
\hline 3 & $\begin{array}{l}\text { goes to another place/ looks another restaurant, which allows informal suit and } \\
\text { plans to visit the new restaurant another time/ dinners at the restaurant bar in casual } \\
\text { suit/ sees if his/her friend has other clothes with him/her or loaned at the restaurant } \\
\text { (Q) (*your friend is dressing jeans and tennis) }\end{array}$ \\
\hline 2 & $\begin{array}{l}\text { Vague answers implying "you" "would away" without specifying going to another } \\
\text { restaurant or whom is included (e.g., "I should go to McDonalds")/ verify what } \\
\text { would be necessary to be appropriately dressed (Q)/ extreme reactions" I am very } \\
\text { angry/I will go away and never come back"/ vague answers as "I would argue this } \\
\text { with my friend and would let him/her decide"/to discuss with the manager }\end{array}$ \\
\hline $\mathbf{1}$ & $\begin{array}{l}\text { speaks to his/her friend who is dressed casual to go home, change clothes and } \\
\text { come back to the restaurant (you would be waiting in the bar of the restaurant) } \\
\text { (*your friend lives far)/ looks a close store and buy something appropriate/ tries to } \\
\text { convince or to bribe the manager to let all of you getting in/ breaks the rule/ } \\
\text { reschedules everything to another night }\end{array}$ \\
\hline $\mathbf{0}$ & $\begin{array}{l}\text { Get in with the appropriately dressed friend and leaves away the friend who is } \\
\text { informally dressed }\end{array}$ \\
\hline
\end{tabular}

11. You are driving in the left track of the road almost at speed limit. You notice that the car behind you is running fast. What would you do?

3 signals and changes track/ lets the car go by $(\mathbf{Q}) /$ keeps a track free/ turn aside the car and lets it goes by or follow through (with the implication to change track but get out of the road)/ answer that bears the objective of moving away from the driver with little effort, consequence or risk - and not involving to get out of the road (*its a 4 tracks road)

2 Lets them go by and calls 911 or calls to ask for help/ speeds up and then signals for the car pass by (slows down or speeds up with the hint to let the car pass by) and pull up to the curb/ turns to the edge of the road or slope of exit

1 answers about moving away from the track with excessiveness or not related to let the car to pass by or being dangerous/ (to get apprehensive and) leaves the road in the next exit/ to drive to the police station if it seems that the car is following you/ to call $911 /$ leave the road in the next available place/ "do anything to try to keep off them"/ to make signal form tem to pass you $(\mathbf{Q}) /$ goes slow until it passes/ steps on the brake abruptly to give a lesson to them and then lets it pass by/ you shouldn't drive in the left track (Q)

0 Does nothing/ I keep driving in current speed, don't bother/ I keep in my pace/ speeds up/ stamps the brake/ steps the brake lightly/ speeds up and then slows down/ puts them against the car next to you / lights the emergency flashers (to make they think that you are stepping on the brake" and the slow down/ opens window glass and waves them to go back/ just keeps going till finds an exit/ "I leave enough space then they will not crash me if I have to stop" 
12. You see a small and friendly dog with a collar alone in the sidewalk in your neighborhood. What would you do?

\begin{tabular}{|l|l|}
\hline 3 & $\begin{array}{l}\text { (tries to make sure if the dog is tame) - then looks at the information in the collar } \\
\text { and looks around for the owner or calls the number in the identification/ looks at } \\
\text { the collar and calls the local animal care or animal shelter/ looks at the collar to } \\
\text { identify the owner/ if you have fear of dogs, calls for human center or calls for help }\end{array}$ \\
\hline 2 & $\begin{array}{l}\text { looks at the collar (without talking about seeing identification or finding the } \\
\text { owner)/ vague answer on trying to find the owner ("I try to identify the dog and to } \\
\text { call the owner") (Q) without mentioning looking at the collar or identification/ } \\
\text { calls the county kennel (Q) }\end{array}$ \\
\hline $\mathbf{1}$ & $\begin{array}{l}\text { an answer that involves to try physically to find the owner without a clear plan or } \\
\text { without looking at the collar (e.g., "I ask for the customers of close stores or people } \\
\text { in the street, if they had lost a dog"/ catch the dog and knock door to door/ takes it } \\
\text { home and does an advertisement/I take it and carry to the veterinarian }\end{array}$ \\
\hline $\mathbf{0}$ & $\begin{array}{l}\text { Nothing, I keep walking/ let it alone/ says "hi" to the dog or talk with the dog and } \\
\text { keep on going }\end{array}$ \\
\hline
\end{tabular}

13. You are diagnosed with a rare illness of blood that causes much discomfort and problems in the brain. Your doctor explains that a new medical procedure can help to cure the illness but this procedure has a mortality rate of $10 \%$. Alternatively, a commonly used medication can provide relief of some symptoms. What would you do?

\begin{tabular}{|l|l|}
\hline 3 & $\begin{array}{l}\text { searches the subject and carefully considers the risks and benefits - and then } \\
\text { decides/ investigates on alternative medicines/ makes many questions (Q)/ gets a } \\
\text { second opinion before deciding/ searches a temporary relief and then a second } \\
\text { opinion }\end{array}$ \\
\hline 2 & $\begin{array}{l}\text { chooses a good treatment with good theoretical base/ chooses a treatment but } \\
\text { recognizes that the other can be a viable option/ tries the medicine first, and if it } \\
\text { doesn't work looks for the cure/ consideration about alternatives but chooses one } \\
\text { without searching further/ follows the advice of the doctor (and takes the } \\
\text { medicine)/ "asks about side effects" (without talking about the need to make a } \\
\text { decision) }\end{array}$ \\
\hline $\mathbf{1}$ & $\begin{array}{l}\text { chooses an option without a rational analysis immediately (e.g., "I try the } \\
\text { medicine" or "I do the operation")/ vague answers "I opt for the safest treatment" } \\
\text { (Q)/"I look for help I can get" (Q)/"which is less expensive for the relatives"/ gets } \\
\text { relatives opinion/ looks for what to do to feel well }\end{array}$ \\
\hline $\mathbf{0}$ & \begin{tabular}{l} 
I don't know/I do nothing \\
\hline
\end{tabular} \\
\hline
\end{tabular}


14. You notice that you have accidentally marked two appointments in the same afternoon (in opposite sides of the city). What would you do?

\begin{tabular}{|l|l|}
\hline 3 & $\begin{array}{l}\text { calls to reschedule one of them (possibly the less important or most flexible one) } \\
\text { and asks for excuses/ cancels one/ calls to explain (* the appointments are } 60 \mathrm{~km} \\
\text { apart approximately") }\end{array}$ \\
\hline 2 & $\begin{array}{l}\text { tries to attend to both shortening a little each/ vague answers (e.g., "I call both, } \\
\text { evaluate their reactions and then decide")/ goes to the closest and later goes to the } \\
\text { other }\end{array}$ \\
\hline $\mathbf{1}$ & goes for only one - ignores the other \\
\hline $\mathbf{0}$ & $\begin{array}{l}\text { He/she doesn't go to anyone - too much pressure/ cancels both/ I give my tickets } \\
\text { for somebody (Q) }\end{array}$ \\
\hline
\end{tabular}

15. Your bank manager tells you that savings accounts profits will decline in the next months significantly, whereas another riskier application profits will increase. At present, you just have money in savings and you have chance to change your investments after knowing this. What would you do?

\begin{tabular}{|l|l|}
\hline 3 & $\begin{array}{l}\text { transfers a little of money from savings to fixed income with the aim to follow the } \\
\text { recommendation but preventing so drastic changes/ answer about someway } \\
\text { changing investments with savings/ checks others opinion and then makes the } \\
\text { change (*other managers agree) }\end{array}$ \\
\hline $\mathbf{2}$ & $\begin{array}{l}\text { vague answers "I follow his opinion because I trust him" "I change to fixed } \\
\text { income" "I try to get financially better" (Q)/ I look for more advice/ I search/ I do } \\
\text { not follow advices blindly, I use my own judgment/ waits until sees evidence of } \\
\text { improvement in fixed income applications before deciding }\end{array}$ \\
\hline $\mathbf{1}$ & $\begin{array}{l}\text { drastic change based in this recommendation (e.g., to transfer all the money from } \\
\text { savings to fixed income/ to take off the money from savings but make no other } \\
\text { banking application - invest all the money in real estate, gold or dollar/ takes off } \\
\text { all the money from savings and does not keep money in bank anymore/ doesn't } \\
\text { change with rational analysis (e.g., I make nothing since you cannot say what it's } \\
\text { going to happen after that, "I would speak to someone else but I'd stay with the } \\
\text { money applied in savings after all") }\end{array}$ \\
\hline $\mathbf{0}$ & I do not know/I do not understand nothing about this/ "nothing" \\
\hline
\end{tabular}

SCORE: Score is the sum of all 15 itens ( $\max =45$ )

TOTAL SCORE $=$ 
ADITIONAL INFORMATION FOR ADMINISTRATION AND SCORING

- When necessary, repeat the question one time to guarantee that the patient understood.

- Copy patient reply literally.

- Score while you carry the test and be SURE to question when necessary: Inquire when (Q) - with the instruction "Talk more about this" or "Explains what you wanted to say".

- If, after being questioned, the participant gives additional information that increases the reply scoring, give the higher scores to it.

Additional information (in bold) can be given if the participant ask directly about the information or give a reply that contradicts this information.

- In question 10, the friend gender must be specified as being the same of the participant.

- If the participant gives two or more answers, ask him/her to choose between them straightaway: "You said $\mathrm{X}, \mathrm{Y}$ and $\mathrm{Z}$, - which is your final reply?"

- If the participant many answers even after questioned, choose the reply that you find that more get the intended reply.

- If the participant says: "this does not apply to me" (e.g., "I don't drive any more") - ask him/her to imagine that it applies (e.g., please, for this question imagine that you still drive"). If even so the participant reveals reluctant or incapable to obtain this perspective, give him/her reply " 0 ".

- As general rule, if the participant gives a reply with characteristics of a reply of 3 points, but of in vague way, gives him 2 points. 
ANEXO H - TOP-J/15-Br

Nome:

Idade:

Sexo:

\section{Teste de Julgamento Prático (TOP-J/15 - Br)}

INSTRUÇÕES: Agora eu gostaria que você me falasse algumas soluções para problemas ou situações cotidianos. Por favor, seja o mais claro possível em suas respostas.

1. Você está de férias longe de casa e percebe que não tem comprimidos controlados suficientes para toda a viagem. Você não pode ficar sem eles e nem voltar para casa. 0 que você faria?

3 procura um médico (ou plano de saúde) local/ liga pro seu médico e pede pra ele enviar uma receita por fax ou correio/ liga para casa e pede para alguém mandar os remédios Sedex/ liga para um médico ou farmácia local (Q) (e consegue uma receita)/ liga para a farmácia onde você costuma comprar e pergunta se eles tem o histórico das suas receitas no computador

2 vai ao médico local/Pronto Socorro/hospital local e pede uma receita ou descreve o problema/ leva os remédios a uma farmácia local e vê se eles conseguem ajudar/ ("você não tem a receita com você)/ pega amostra grátis ou receita em um médico ou farmácia/ convence o farmacêutico a te dar pílulas suficientes

1 toma doses menores para durarem mais/ economiza-os (raciona-os)/ respostas vagas mesmo após questionado (ex. "vou à farmácia e pego", "ligo para casa e peço ajuda", "consigo que um farmacêutico envie por correio", "consigo algum")

0 espera para ver se você precisa deles, não há motivo para se preocupar adiantado/ nada, espero até chegar em casa/ espera até alguma coisa ruim acontecer/ não sei, continuo sem/ evito esforço físico

2. Você recebe um telefonema avisando que acabou de ganhar um carro em um sorteio do qual participou muitos meses antes. A pessoa que ligou pede para você fornecer seu endereço, CPF e número do cartão de crédito. $\underline{O}$ que você faria?

3 não fornece informações e pede para enviarem um documento por escrito declarando termos e condições do sorteio/ pede uma comprovação da autenticidade por escrito antes de fornecer informações/ fornece o endereço para que possam enviar-lhe comprovação ou checa com o Serviço de Proteção ao Consumidor

2 desliga/ não fornece informação alguma (mas não solicita informações adicionais/ esclarecimento ou vaga referência sobre reunir mais informações - ex., "eles necessitam provar isso para mim")/ não interessado/ não fornece informações por telefone/ tento pegar identificação deles $(\mathbf{Q})$ / pede o número deles e liga de volta para conferir (*o patrocinador do sorteio não é local)/ não dá informações mas faz perguntas/ avisa a polícia (Q)

1 pede mais informações por telefone e depois fornece as informações solicitadas (alguma comprovação é pedida, mas não por escrito)/ somente fornece o endereço (Q)

0 fornece informações (não solicita informações ou esclarecimentos posteriores de forma alguma)/ somente fornece endereço e CPF

Test of Practical Judgment (TOP-J)* adaptado

*Rabin LA, Borgos MJ, Saykin AJ, Wishart HA, Crane PK, Nutter-Upham KE et al. Judgment in older adults: Development and psychometric evaluation of the Test of Practical Judgment (TOP-J). J Clin Exp Neuropsychol. 2007;29:752-67. 
3. Você está fora da cidade visitando parentes e de repente lembra que pode ter deixado o fogão da sua casa ligado. $\underline{O}$ que você faria?

\begin{tabular}{|l|l|}
\hline $\mathbf{3}$ & $\begin{array}{l}\text { entra em contato com alguém que possa ir na sua casa checar (ex. vizinho, proprietário, membro da } \\
\text { família) }\end{array}$ \\
\hline $\mathbf{2}$ & liga para a polícia ou bombeiro \\
\hline $\mathbf{1}$ & pânico/ corre para casa ( você está longe de casa) \\
\hline $\mathbf{0}$ & faz nada/ não sabe \\
\hline
\end{tabular}

4. Você leu uma notícia de que o governo vai reduzir mensalmente os pagamentos da aposentadoria do INSS de $\mathrm{R} \$ 1500,00$ para $\mathbf{R} \$ 750,00$ para uma determinada porcentagem de pensionistas. 0 que você faria?

3 tenta descobrir a probabilidade dos seus pagamentos serem reduzidos/ liga para obter mais informações na tentativa de determinar se isso afeta você/ ligar para o INSS para saber mais (Q) - se você será afetado

2 Tenta vagamente obter mais informações sem tentar determinar diretamente se você será afetado ou supor que será afetado (ex. "eu verifico, porque isso não está certo" "determino a validade da informação" "ligo e vejo o que posso fazer (Q)"/ liga para uma autoridade responsável para obter informação $(\mathbf{Q})$ / pesquisa o problema ou "procura o motivo" (sem averiguar se os benefícios irão mudar)/ pesquisa quanto de redução haverá/ procuro saber se vou ser afetado e se eu for, entro na justiça

1 reduz os gastos mensais/ deixa as contas pagas para manter o orçamento controlado/ vai trabalhar/ pede dinheiro emprestado/ ajusta as finanças/ entro na justiça

0 faz nada/ espera para ver o que vai acontecer/ isso não me afeta/ não se pode brigar com o governo (Q)/ diz para o governo que isso é uma má idéia/ reclama ou liga para jornais locais/ não acredito nisso/ eu só vivo com meus recursos/ escrevo para o meu senador/deputado e me queixo/ fico bravo

5. Você está em um jantar na casa de um amigo, mas o prato principal está com gosto de estragado. $\underline{O}$ que você faria?

3 para de comer e avisa o anfitrião discretamente/ avalia a reação das pessoas e diz alguma coisa para o anfitrião (querendo dizer que é melhor parar de comer ou que algo está errado)

2 para de comer (mas sem falar para o anfitrião ou outros convidados)/ come os acompanhamentos/ separa a parte ruim/ fala para o anfitrião e para os outros "isto está com o gosto ruim para mim, está bom para vocês?" (Q)/ pede para o anfitrião provar/ fala alto para os outros convidados (sem falar primeiro para o anfitrião)

1 respostas vagas como empurrar a comida ao redor do prato/ outras respostas vagas (Q)/ pergunta sobre os ingredientes/ mente para os outros (ex. "Eu sou alérgico") ou propositalmente desvia a atenção da comida

0 continua comendo/ faz nada - não queria ofender um amigo/ se vira com o que for possível (Q)/ somente come um pouco 
6. Você toma uma medicação diária para reduzir o colesterol, mas um estudo novo publicado no jornal local sugere que esta medicação está relacionada a um alto risco para câncer. 0 que você faria?

3 procura saber mais sobre o estudo (ex. como a pesquisa foi conduzida, se outros dados apóiam os achados, que tipo de câncer)/ liga para um especialista/ liga para seu médico/ pergunta sobre medicamentos alternativos

2 tenta passivamente discernir a validade do estudo (ex. "espero por mais confirmações"/ "ignoro isso até que seja verificado por outro estudo")

1 para de tomar medicação e depois obtém maiores informações do médico ou sites médicos/ para de tomar a medicação/ fala para o médico e insiste em parar de tomar a medicação/ continua tomando a medicação (com boa justificativa)

0 ignora os achados/ faz nada/ ri da publicação - isso é impossível/ continua tomando a medicação (com justificativa ruim - ex. "Eu sou velho, quem se importa se eu tiver câncer")

7. Você está começando a ter problemas para dirigir devido a problemas na visão noturna e discreta confusão mental. $\underline{O}$ que você faria?

3 Faz uma consulta com um médico para determinar a causa do problema (e para de dirigir a noite até que a causa seja determinada/ enquanto espera a opinião do médico)

2 evita dirigir a noite/ somente dirige para lugares que você conhece bem/ pede para esposa ou amigo dirigir a noite/ vaga referência sobre saber mais sobre o problema como "ir ao médico" (Q) ou "peço para minha esposa me supervisionar e deixo ela dirigir mais"

1 desiste completamente de dirigir/ deixar de dirigir (sem menção de estar sendo avaliado)/ discutir com a família e desistir de dirigir/ "Eu não dirijo" (Q)/ ter alguém para dirigir para $\mathrm{mim} /$ "segurança vem primeiro, mas eu não tenho certeza"

0 faz nada/ "não saio de casa a noite" (Q)/ saio somente se não estiver chovendo

8. Você acha uma carteira na calçada. Você a pega e 5 passos depois você acha uma nota de RS 50,00 . O que você faria?

3 qualquer resposta que busque determinar quem é o dono ou devolver ambos a carteira e o dinheiro e reconhecer a possibilidade de que não pertençam à mesma pessoa/ levar a carteira à polícia ou prefeitura e explicar sobre a nota/ procurar o RG na carteira e ligar para a pessoa para verificar a quantia na carteira (e devolver ambos)

2 qualquer resposta que busque tentar determinar o dono da carteira e devolver a carteira, mas não a nota que possivelmente não estava na carteira (ex. procurar o RG na carteira e tentar encontrar o dono)/ levá-la à polícia/ qualquer resposta dizendo que a carteira e a nota deveriam ser devolvidas a um único dono/ leva a carteira ou ambos à polícia

1 leva a carteira para a delegacia mas fica com a nota/ "devolvo a carteira, e se eu gostar da pessoa devolvo a nota, do contrário fico com ela"/ fica com a nota mas tenta devolver a carteira

0 fica com ambos/ somente continua andando/ "eu pegaria e sairia correndo - achando que é uma armadilha" 
9. Você foi dirigindo a um shopping em uma cidade vizinha, e quando acabou de fazer as compras estava escuro lá fora. Seu carro está parado a uma grande distância da saída e lá tem acontecido muitos assaltos recentemente. $\underline{O}$ que você faria?

3 acha um segurança ou policial e pede a eles para acompanhá-lo ao seu carro/ avalia a segurança da situação e encontra alguém para acompanhá-lo até o carro ou trazer o carro para você/ (*você foi ao shopping sozinho)

2 liga para um familiar ou amigo para que o peguem/ caminha ao lado de outro (s) freguês (s)/ chama um taxi

1 vai para o carro mas fica atento a algo suspeito/ caminha até o carro mas segura a chave nas mãos como um artifício de proteção/ caminha até o carro mas mantém o olhar no segurança/ caminha até o carro mas mantém o celular próximo

0 "caminho até o carro" (sem reconhecimento que a situação possa ser insegura)/ "eu não me preocupo com o ataque de outras pessoas"/ não sabe/ entra em pânico/ isso nunca aconteceria comigo/ "não estou preocupado"/ é uma má idéia ir para uma área como essa/ grita, chama a atenção e bate neles com alguma coisa

10. Você se encontra com dois amigos íntimos para um jantar em um restaurante que você está muito empolgado para conhecer. Entretanto, na chegada o gerente explica que um dos seus amigos não pode entrar porque ele/ela está vestido de forma muito casual. 0 que você faria?

3 vai para outro lugar/ procura um outro restaurante, que permita traje informal e planeja visitar o restaurante novo outra hora/ janta no bar do restaurante em traje casual/ vê se seu amigo tem outras roupas com ele ou se alguém do restaurante pode emprestar (Q) ("seu amigo está vestindo jeans e tênis)

2 Resposta vaga indicando "você" "iria embora" sem especificar ir para outro restaurante ou quem está incluído (ex. "Eu deveria ir para o Mcdonalds")/ verificar o que seria necessário para ele ficar apropriadamente vestido $(\mathbf{Q})$ / reações extremas"fico muito bravo/vou embora e nunca mais volto"/ vaga resposta como "Eu discutiria isso com meu amigo e deixaria ele decidir"/ discutir com o gerente

1 fala para o seu amigo que está vestido casualmente ir para casa, mudar de roupa e voltar para o restaurante (você ficará esperando no bar do restaurante) (“seu amigo mora longe)/ procura uma loja próxima e compra algo apropriado/ tenta convencer ou subornar o gerente para deixar todos vocês entrarem/ quebra a regra/ reprograma tudo para uma outra noite

0 entra com o amigo vestido apropriadamente e deixa o amigo vestido informalmente para trás 
11. Você está dirigindo na estrada na pista da esquerda quase na velocidade limite. Você repara que o carro atrás de você está correndo. $\underline{O}$ que você faria?

3 dá sinal e muda de pista/ deixa o carro passar (Q)/ dá lugar em uma pista/ desvia o carro e o permite passar ou seguir em frente (com a implicação de mudar de pista mas não fícar inteiramente fora da estrada)/ resposta que conclui o objetivo de se afastar do motorista com pouco esforço, conseqüência ou risco - e não envolver físicamente sair da estrada ("é uma estrada de 4 pistas)

2 deixa eles passarem e liga 192 ou liga para pedir ajuda/ acelera e depois dá sinal para o carro passar/ (desacelerar ou acelerar com a insinuação de deixar o carro passar) e encosta no meio fio-desvia para a margem da estrada ou rampa de saída

1 respostas que envolvam se afastar da pista com exagero ou não envolvam deixar o carro passar ou é perigoso/ (ficar nervoso e) sai da estrada na próxima saída/ dirigir até a delegacia se parecer que o carro está seguindo você/ ligar 192/ sai da estrada no ponto disponível mais próximo/ "faço qualquer coisa para tentar se desviar deles"/ fazer sinal para eles passarem você (Q)/ vai devagar até que ele passe/ pisa no freio momentaneamente para dar-lhes uma lição e depois deixa que ele passe/ você não deveria dirigir na pista da esquerda (Q)

0 Faz nada/ continuo dirigindo na velocidade atual, não se preocupa/ continuo no meu ritmo/ acelera/ pisa no freio com força/ pisa no freio levemente/ acelera e depois desacelera/ coloca-os contra o carro próximo a você/ liga o "pisca-alerta" (para fazer eles pensarem que você está pisando no freio" e depois desacelera/ abre o vidro e acena para eles irem para trás/ somente continua andando até você achar uma saída/ "deixo espaço suficiente então eles não irão bater em mim se eu tiver que parar"

12. Você vê um cachorro pequeno e amigável, com uma coleira, sozinho na calçada na sua vizinhança. $\underline{O}$ que você faria?

3 (tenta ter certeza se o cachorro é manso) - depois olha a informação na coleira e procura ao redor pelo dono ou liga no número que está na identificação/ olha a coleira e liga para a carrocinha ou abrigo de animais/ olha na coleira para identificar o dono/ se você tem medo de cães, liga para abrigo de cães abandonados ou liga para ajuda

2 olha para a coleira (sem falar de ver identificação ou encontrar o proprietário)/ resposta vaga sobre tentar localizar o dono ("tento identificar o cachorro e ligar para o dono") (Q) sem mencionar olhar a coleira ou identificação/ liga para canil municipal (Q)

1 uma resposta que envolva tentar fisicamente localizar o proprietário sem um plano claro ou sem olhar para a coleira (ex. "pergunto para os clientes de lojas próximas ou pessoas próximas na rua, se eles perderam um cachorro"/ pega o cachorro e bate de porta em porta/ leva ele para casa e faz um anúncio/ pego ele e levo ao veterinário/

0 Nada, continuo andando/ deixa ele sozinho/ diz "oi" para o cachorro ou fala com o cachorro e prossegue 
13. Você está diagnosticado com uma rara doença de sangue que causa muito desconforto e problemas no cérebro. Seu médico explica que um novo procedimento médico pode ajudar a curar a doença, mas esse procedimento tem uma taxa de mortalidade de $10 \%$. Alternativamente, uma medicação comumente usada pode proporcionar alívio de alguns sintomas. $\underline{O}$ que você faria?

3 pesquisa o assunto e cuidadosamente considera os riscos e benefícios - e então decide/ investiga sobre medicamentos alternativos/ faz muitas perguntas $(\mathbf{Q}) /$ pega uma segunda opinião antes de decidir/ procura um alívio temporário e então uma segunda opinião

2 escolhe um bom tratamento com boa base teórica/ escolhe um tratamento mas reconhece que o outro possa ser uma opção viável/ tenta o remédio primeiro, e se não funcionar procura a cura/ consideração sobre alternativas mas escolhe um sem pesquisar mais/ segue o conselho do médico (e toma o remédio)/ "questiona sobre os efeitos colaterais"(sem falar da necessidade de tomar uma decisão)

1 escolhe imediatamente uma opção sem uma análise racional (ex. "tento a medicação" ou "faço a operação")/ respostas vagas "opto pelo tratamento mais seguro" $(\mathbf{Q})$ / "vou atrás do auxílio que eu puder obter" (Q)/ "o que for menos caro para os parentes"/ pega opinião de parentes/ procura o que fazer para ficar bem

0 não sei/ faço nada

14. Você percebe que acidentalmente marcou dois compromissos na mesma tarde (em lados opostos da cidade). O que você faria?

3 liga para remarcar um deles (possivelmente aquele menos importante ou o mais flexível) e pede desculpas/ cancela um/ liga para explicar (* os compromissos são aproximadamente $60 \mathrm{Km}$ de distância")

2 tenta cumprir os dois reduzindo um pouco de cada/ respostas vagas (ex. "ligo para ambos, avalio as reações e depois decido")/ vai para o mais perto e depois vai para o outro

1 vai somente para um - ignora o outro

0 não vai para nenhum - pressão demais/ cancela ambos/ dou meus ingressos para alguém (Q)

15. O gerente do seu banco avisa que os ganhos com a caderneta de poupança irão declinar significativamente nos próximos meses, enquanto que os ganhos com outra aplicação que tem risco irão aumentar. Atualmente, você só tem dinheiro na poupança e tem oportunidade de mudar seus investimentos após este aviso. $\underline{\text { O que você faria? }}$

3 transfere um pouco de dinheiro da poupança para a aplicação com risco, com objetivo de seguir a recomendação mas evitar mudança tão drástica/ resposta que envolva mudar os investimentos com poupança de alguma forma/ procura opinião de outros e depois faz a mudança (*outros gerentes concordam)

2 respostas vagas "sigo a opinião porque eu confio nele" "mudo para a aplicação com risco" "tento ficar melhor financeiramente" $(\mathbf{Q})$ / procuro mais conselhos / pesquiso / não sigo conselhos às cegas, uso meu próprio julgamento / espera até você ver evidência de melhora nas aplicações de risco antes de decidir

1 mudança drástica baseada nessa recomendação (ex. transferir todo o dinheiro da poupança para a aplicação com risco/ tirar o dinheiro da poupança mas não fazer mais nenhuma aplicação bancária - investe todo o dinheiro em imóveis, ouro ou dólar/ tirar todo o dinheiro da poupança e não guardar mais dinheiro em banco/ não muda com análise racional (ex. faço nada já que você não pode me dizer o que vai acontecer em seguida, "eu consultaria mais alguém mas no final ficaria com o dinheiro aplicado em poupança")

0 não sei/ não entendo nada sobre isso/ "nada" 
ESCORE: O escore é a soma de todos os 15 itens $(\max =45)$

\section{ESCORE TOTAL =}

\section{INFORMAÇÕES ADICIONAIS PARA ADMINISTRAÇÃO E PONTUAÇÃ̃o}

- Quando necessário, repita a questão uma vez para garantir que o paciente entendeu.

- Anotar a resposta do paciente, literalmente.

- Pontue enquanto realiza o teste e esteja SEGURO para questionar quando necessário: Questione quando houver $(Q)$ - com a instrução "Fale mais sobre isso" ou "Explique o que você quis dizer".

- Se, após ser questionado, o participante der informações adicionais que aumentem a pontuação da resposta, dê a ele/ela o valor mais alto.

- Informações adicionais (em negrito) podem ser dadas se o participante perguntar diretamente sobre a informação ou der uma resposta que contradiga essa informação.

- Na questão 10, o gênero do amigo deve ser especificado como sendo o mesmo do participante.

- Se o participante der duas ou mais respostas, peça pra ele/ela escolher entre elas de forma direta: "Você disse $\mathrm{X}, \mathrm{Y}$ e Z, - qual é a sua resposta final?"

- Se o participante dá muitas respostas mesmo depois de questionado, escolha a resposta que você acha que mais capta a resposta pretendida.

- Se o participante diz: "isso não se aplica a mim" (ex. "Eu não dirijo mais") - peça para ele/ela imaginar que se aplica (ex. para esta questão, por favor imagine que você ainda dirige"). Se mesmo assim o participante mostrar-se relutante ou incapaz de conseguir esta perspectiva, dê a ele/ela resposta " 0 ".

- Como regra geral, se o participante dá uma resposta com características de uma resposta de 3 pontos, mas de forma vaga, dê a ele 2 pontos.

Média dos Controles: $33,4 \pm 4$

Pontos de Corte

Controle x CCL: 31

Controle X demência: 30 
ANEXO I - TOP-J/9-Br

Nome:

Idade:

Data:

Sexo:

\title{
Teste de Julgamento Prático (TOP-J/9 - Br)
}

\author{
INSTRUÇÕES: Agora eu gostaria que você me falasse algumas soluções para problemas ou \\ situações cotidianos. Por favor, seja o mais claro possível em suas respostas.
}

1. Você está de férias longe de casa e percebe que não tem comprimidos controlados suficientes para toda a viagem. Você não pode ficar sem eles e nem voltar para casa. 0 que você faria?
3 procura um médico (ou plano de saúde) local/ liga pro seu médico e pede pra ele enviar uma receita por fax ou correio/ liga para casa e pede para alguém mandar os remédios Sedex/ liga para um médico ou farmácia local (Q) (e consegue uma receita)/ liga para a farmácia onde você costuma comprar e pergunta se eles tem o histórico das suas receitas no computador
2 vai ao médico local/Pronto Socorro/hospital local e pede uma receita ou descreve o problema/ leva os remédios a uma farmácia local e vê se eles conseguem ajudar/ ("você não tem a receita com você)/ pega amostra grátis ou receita em um médico ou farmácia/ convence o farmacêutico a te dar pílulas suficientes
1 toma doses menores para durarem mais/ economiza-os (raciona-os)/ respostas vagas mesmo após questionado (ex. "vou à farmácia e pego", "ligo para casa e peço ajuda", "consigo que um farmacêutico envie por correio", "consigo algum")
0 espera para ver se você precisa deles, não há motivo para se preocupar adiantado/ nada, espero até chegar em casa/ espera até alguma coisa ruim acontecer/ não sei, continuo sem/ evito esforço físico

2. Você recebe um telefonema avisando que acabou de ganhar um carro em um sorteio do qual participou muitos meses antes. A pessoa que ligou pede para você fornecer seu endereço, CPF e número do cartão de crédito. O que você faria?

3 não fornece informações e pede para enviarem um documento por escrito declarando termos e condições do sorteio/ pede uma comprovação da autenticidade por escrito antes de fornecer informações/ fornece o endereço para que possam enviar-lhe comprovação ou checa com o Serviço de Proteção ao Consumidor

2 desliga/ não fornece informação alguma (mas não solicita informações adicionais/ esclarecimento ou vaga referência sobre reunir mais informações - ex., "eles necessitam provar isso para mim")/ não interessado/ não fornece informações por telefone/ tento pegar identificação deles $(\mathbf{Q}) /$ pede o número deles e liga de volta para conferir (*o patrocinador do sorteio não é local)/ não dá informações mas faz perguntas/avisa a polícia (Q)

1 pede mais informações por telefone e depois fornece as informações solicitadas (alguma comprovação é pedida, mas não por escrito)/ somente fornece o endereço (Q)

0 fornece informações (não solicita informações ou esclarecimentos posteriores de forma alguma)/ somente fornece endereço e CPF

Test of Practical Judgment (TOP-J)* adaptado

*Rabin LA, Borgos MJ, Saykin AJ, Wishart HA, Crane PK, Nutter-Upham KE et al. Judgment in older adults: Development and psychometric evaluation of the Test of Practical Judgment (TOP-J). J Clin Exp Neuropsychol. 2007;29:752-67. 
3. Você leu uma notícia de que o governo vai reduzir mensalmente os pagamentos da aposentadoria do INSS de R\$1500,00 para R\$750,00 para uma determinada porcentagem de pensionistas. $\underline{O}$ que você faria?

\begin{tabular}{|l|l|}
\hline $\mathbf{3}$ & $\begin{array}{l}\text { tenta descobrir a probabilidade dos seus pagamentos serem reduzidos/ liga para obter mais informações na } \\
\text { tentativa de determinar se isso afeta você/ ligar para o INSS para saber mais (Q) - se você será afetado }\end{array}$ \\
\hline $\mathbf{2}$ & $\begin{array}{l}\text { Tenta vagamente obter mais informações sem tentar determinar diretamente se você será afetado ou supor } \\
\text { que será afetado (ex. "eu verifico, porque isso não está certo" "determino a validade da informação" "ligo e } \\
\text { vejo o que posso fazer (Q)"/ liga para uma autoridade responsável para obter informação (Q)/ pesquisa o } \\
\text { problema ou "procura o motivo" (sem averiguar se os benefícios irão mudar)/ pesquisa quanto de redução } \\
\text { haverá/ procuro saber se vou ser afetado e se eu for, entro na justiça }\end{array}$ \\
\hline $\mathbf{1}$ & $\begin{array}{l}\text { reduz os gastos mensais/ deixa as contas pagas para manter o orçamento controlado/ vai trabalhar/ pede } \\
\text { dinheiro emprestado/ ajusta as finanças/ entro na justiça }\end{array}$ \\
\hline $\mathbf{0}$ & $\begin{array}{l}\text { faz nada/ espera para ver o que vai acontecer/ isso não me afeta/ não se pode brigar com o governo (Q)/ diz } \\
\text { para o governo que isso é uma má idéia/ reclama ou liga para jornais locais/ não acredito nisso/ eu só vivo } \\
\text { com meus recursos/ escrevo para o meu senador/deputado e me queixo/ fico bravo }\end{array}$ \\
\hline
\end{tabular}

4. Você toma uma medicação diária para reduzir o colesterol, mas um estudo novo publicado no jornal local sugere que esta medicação está relacionada a um alto risco para câncer. $\underline{\text { O que você faria? }}$

\begin{tabular}{|c|l|}
\hline $\mathbf{3}$ & $\begin{array}{l}\text { procura saber mais sobre o estudo (ex. como a pesquisa foi conduzida, se outros dados apóiam os achados, } \\
\text { que tipo de câncer)/ liga para um especialista/ liga para seu médico/ pergunta sobre medicamentos } \\
\text { alternativos }\end{array}$ \\
\hline $\mathbf{2}$ & $\begin{array}{l}\text { tenta passivamente discernir a validade do estudo (ex. "espero por mais confirmações"/ "ignoro isso até que } \\
\text { seja verificado por outro estudo") }\end{array}$ \\
\hline $\mathbf{1}$ & $\begin{array}{l}\text { para de tomar medicação e depois obtém maiores informações do médico ou sites médicos/ para de tomar a } \\
\text { medicação/ fala para o médico e insiste em parar de tomar a medicação/ continua tomando a medicação } \\
\text { (com boa justificativa) }\end{array}$ \\
\hline $\mathbf{0}$ & $\begin{array}{l}\text { ignora os achados/ faz nada/ ri da publicação - isso é impossível/ continua tomando a medicação (com } \\
\text { justificativa ruim - ex. "Eu sou velho, quem se importa se eu tiver câncer") }\end{array}$ \\
\hline
\end{tabular}

5. Você está começando a ter problemas para dirigir devido a problemas na visão noturna e discreta confusão mental. $\underline{O}$ que você faria?

\begin{tabular}{|l|l|}
\hline $\mathbf{3}$ & $\begin{array}{l}\text { Faz uma consulta com um médico para determinar a causa do problema (e para de dirigir a noite até que a } \\
\text { causa seja determinada/ enquanto espera a opinião do médico) }\end{array}$ \\
\hline $\mathbf{2}$ & $\begin{array}{l}\text { evita dirigir a noite/ somente dirige para lugares que você conhece bem/ pede para esposa ou amigo dirigir a } \\
\text { noite/ vaga referência sobre saber mais sobre o problema como "ir ao médico" (Q) ou "peço para minha } \\
\text { esposa me supervisionar e deixo ela dirigir mais" }\end{array}$ \\
\hline $\mathbf{1}$ & $\begin{array}{l}\text { desiste completamente de dirigir/ deixar de dirigir (sem menção de estar sendo avaliado)/ discutir com a } \\
\text { família e desistir de dirigir/ "Eu não dirijo" (Q)/ ter alguém para dirigir para mim/ "segurança vem primeiro, } \\
\text { mas eu não tenho certeza" }\end{array}$ \\
\hline $\mathbf{0}$ & faz nada/ "não saio de casa a noite" (Q)/ saio somente se não estiver chovendo \\
\hline
\end{tabular} 
6. Você acha uma carteira na calçada. Você a pega e 5 passos depois você acha uma nota de $\mathrm{R} \$ \mathbf{5 0 , 0 0}$. O que você faria?

\begin{tabular}{|l|l|}
\hline 3 & $\begin{array}{l}\text { qualquer resposta que busque determinar quem é o dono ou devolver ambos a carteira e o dinheiro e } \\
\text { reconhecer a possibilidade de que não pertençam à mesma pessoa/ levar a carteira à polícia ou prefeitura e } \\
\text { explicar sobre a nota/ procurar o RG na carteira e ligar para a pessoa para verificar a quantia na carteira (e } \\
\text { devolver ambos) }\end{array}$ \\
\hline $\mathbf{2}$ & $\begin{array}{l}\text { qualquer resposta que busque tentar determinar o dono da carteira e devolver a carteira, mas não a nota que } \\
\text { possivelmente não estava na carteira (ex. procurar o RG na carteira e tentar encontrar o dono)/ levá-la à } \\
\text { polícia/ qualquer resposta dizendo que a carteira e a nota deveriam ser devolvidas a um único dono/ leva a } \\
\text { carteira ou ambos à polícia }\end{array}$ \\
\hline $\mathbf{1}$ & $\begin{array}{l}\text { leva a carteira para a delegacia mas fica com a nota/ "devolvo a carteira, e se eu gostar da pessoa devolvo a } \\
\text { nota, do contrário fico com ela"/ fica com a nota mas tenta devolver a carteira }\end{array}$ \\
\hline $\mathbf{0}$ & fíca com ambos/ somente continua andando/ "eu pegaria e sairia correndo - achando que é uma armadilha" \\
\hline
\end{tabular}

7. Você se encontra com dois amigos íntimos para um jantar em um restaurante que você está muito empolgado para conhecer. Entretanto, na chegada o gerente explica que um dos seus amigos não pode entrar porque ele/ela está vestido de forma muito casual. $\underline{O}$ que você faria?

\begin{tabular}{|l|l|}
\hline 3 & $\begin{array}{l}\text { vai para outro lugar/ procura um outro restaurante, que permita traje informal e planeja visitar o restaurante } \\
\text { novo outra hora/ janta no bar do restaurante em traje casual/ vê se seu amigo tem outras roupas com ele ou se } \\
\text { alguém do restaurante pode emprestar (Q) ("seu amigo está vestindo jeans e tênis) }\end{array}$ \\
\hline $\mathbf{2}$ & $\begin{array}{l}\text { Resposta vaga indicando "você" "iria embora" sem especificar ir para outro restaurante ou quem está incluído } \\
\text { (ex. "Eu deveria ir para o Mcdonalds")/ verificar o que seria necessário para ele ficar apropriadamente } \\
\text { vestido (Q)/ reações extremas"fico muito bravo/vou embora e nunca mais volto"/ vaga resposta como "Eu } \\
\text { discutiria isso com meu amigo e deixaria ele decidir"/ discutir com o gerente }\end{array}$ \\
$\mathbf{1}$ & $\begin{array}{l}\text { fala para o seu amigo que está vestido casualmente ir para casa, mudar de roupa e voltar para o restaurante } \\
\text { (você ficará esperando no bar do restaurante) ("seu amigo mora longe)/ procura uma loja próxima e compra } \\
\text { algo apropriado/ tenta convencer ou subornar o gerente para deixar todos vocês entrarem/ quebra a regra/ } \\
\text { reprograma tudo para uma outra noite }\end{array}$ \\
\hline $\mathbf{0}$ & entra com o amigo vestido apropriadamente e deixa o amigo vestido informalmente para trás \\
\hline
\end{tabular}

8. Você vê um cachorro pequeno e amigável, com uma coleira, sozinho na calçada na sua vizinhança. $\underline{\mathrm{O}}$ que você faria?

3 (tenta ter certeza se o cachorro é manso) - depois olha a informação na coleira e procura ao redor pelo dono ou liga no número que está na identificação/ olha a coleira e liga para a carrocinha ou abrigo de animais/ olha na coleira para identificar o dono/ se você tem medo de cães, liga para abrigo de cães abandonados ou liga para ajuda

2 olha para a coleira (sem falar de ver identificação ou encontrar o proprietário)/ resposta vaga sobre tentar localizar o dono ("tento identificar o cachorro e ligar para o dono") (Q) sem mencionar olhar a coleira ou identificação/ liga para canil municipal (Q)

1 uma resposta que envolva tentar físicamente localizar o proprietário sem um plano claro ou sem olhar para a coleira (ex. "pergunto para os clientes de lojas próximas ou pessoas próximas na rua, se eles perderam um cachorro"/ pega o cachorro e bate de porta em porta/ leva ele para casa e faz um anúncio/ pego ele e levo ao veterinário/

0 Nada, continuo andando/ deixa ele sozinho/ diz "oi" para o cachorro ou fala com o cachorro e prossegue 
9. Você percebe que acidentalmente marcou dois compromissos na mesma tarde (em lados opostos da cidade). 0 que você faria?

\begin{tabular}{|l|l|}
\hline $\mathbf{3}$ & $\begin{array}{l}\text { liga para remarcar um deles (possivelmente aquele menos importante ou o mais flexível) e pede desculpas/ } \\
\text { cancela um/ liga para explicar (* os compromissos são aproximadamente } 60 \mathrm{Km} \text { de distância") }\end{array}$ \\
\hline $\mathbf{2}$ & $\begin{array}{l}\text { tenta cumprir os dois reduzindo um pouco de cada/ respostas vagas (ex. "ligo para ambos, avalio as reações e } \\
\text { depois decido")/ vai para o mais perto e depois vai para o outro }\end{array}$ \\
\hline $\mathbf{1}$ & vai somente para um - ignora o outro \\
\hline $\mathbf{0}$ & não vai para nenhum - pressão demais/ cancela ambos/ dou meus ingressos para alguém (Q) \\
\hline
\end{tabular}

ESCORE: O escore é a soma de todos os 9 itens $(\max =27)$

ESCORE TOTAL $=$

\section{INFORMAÇÕES ADICIONAIS PARA ADMINISTRAÇÃO E PONTUACุÃO}

- Quando necessário, repita a questão uma vez para garantir que o paciente entendeu.

- Anotar a resposta do paciente, literalmente.

- Pontue enquanto realiza o teste e esteja SEGURO para questionar quando necessário: Questione quando houver $(\mathrm{Q})$ - com a instrução "Fale mais sobre isso" ou "Explique o que você quis dizer".

- Se, após ser questionado, o participante der informações adicionais que aumentem a pontuação da resposta, dê a ele/ela o valor mais alto.

- Informações adicionais (em negrito) podem ser dadas se o participante perguntar diretamente sobre a informação ou der uma resposta que contradiga essa informação.

- Se o participante der duas ou mais respostas, peça pra ele/ela escolher entre elas de forma direta: "Você disse X, Y e Z, - qual é a sua resposta final?"

- Se o participante dá muitas respostas mesmo depois de questionado, escolha a resposta que você acha que mais capta a resposta pretendida.

- Se o participante diz: "isso não se aplica a mim" (ex. "Eu não dirijo mais") - peça para ele/ela imaginar que se aplica (ex. para esta questão, por favor imagine que você ainda dirige"). Se mesmo assim o participante mostrar-se relutante ou incapaz de conseguir esta perspectiva, dê a ele/ela resposta " 0 ".

- Como regra geral, se o participante dá uma resposta com características de uma resposta de 3 pontos, mas de forma vaga, dê a ele 2 pontos.

Média dos Controles: 20,2 $\pm 2,4$

Pontos de Corte

Controle x CCL: 19

Controle X demência: 18 


\section{REFERÊNCIAS}

Allaire JC, Marsiske M. Everyday Cognition: Age and Intellectual Ability Correlates. Psychol Aging. 1999;14(4):627-44.

Almeida OP, Almeida SA. Short Versions of the Geriatric Depression Scale: A Study of their validity for the diagnosis of a major depressive episode according to ICD-10 and DSM-IV. Int J Geriat Psychiatry. 1999;14:858-65.

American Psychiatric Association. Diagnostic and Statistical Manual of Mental Disorders. $3^{\text {a }}$ ed. Rev. Washington, DC: American Psychiatric Association, 1987.

Armentano CGC, Porto CS, Nitrini R, Brucki SMD. Ecological Evaluation of Executive Functions in Mild Cognitive Impairment and Alzheimer Disease. Alzheimer Dis Assoc Disord. 2013;27(2):95-101.

Artistico D, Cervone D, Pezzuti L. Perceived self-efficacy and everyday problem solving among young and older adults. Psychol Aging. 2003;18:68-79.

Ávila KN. Modified Wisconsin Card Sorting Test (MCST): Desempenho de idosos com diferentes níveis de escolaridade. Psicol Hosp. 2007;5(2):21-35.

Baird A. Fine tuning recommendations for older adults with memory complaints: using the Independent Living Scales with the Dementia Rating Scale. Clin Neuropsychol. 2006;20:649-61.

Baldock D, Miller J, Banks S. Difference between Patients with AD and Patients with FTD on a Test of Practical Judgment. Arch Clin Neuropsychol. 2014;29(6):512. (Presented at the 34th Annual Conference of the National Academy of Neuropsychology, Fajardo, Puerto Rico, 12-15 November 2014). 
Baughman, B.C., Young, J.C., Crouse, E.M., Yehyawi, N.T., Dowd, A., \& Hennessey, L.K. (2011, February). Exploring judgment in geriatric veterans: Factor structure of the Test of Practical Judgment (TOP-J). (Presented at the 39th annual meeting for the International Neuropsychological Society; 2011; Boston, MA).

Benze BG. Pesquisa em Educação Especial: O Desafio da Qualificação. Bauru: EDUSC; 2001. Cap. 2, p 31-45: Metodologia Científica e Estatística.

Bottino CMC, Azevedo D, Tatsch M, Hototian SR, Moscoso MA, Folquitto J, et al. Estimate of dementia prevalence in a community sample from São Paulo, Brazil. Dement Geriatr Cogn Disord. 2008;26(4):291-99.

Brand M, Markowitsch HJ. Aging and Decision-Making: A neurocognitive Perspective. Gerontology. 2010;56:319-24.

Brucki SMD, Malheiros SMF, Okamoto IH, Bertolucci PHF. Dados normativos para o teste de fluência verbal: categoria animais em nosso meio. Arq Neuropsiquiatr. 1997;55(1):56-61.

Brucki SMD, Nitrini R, Caramelli P, Bertolucci PHF, Okamoto IH. Suggestions of utilization of the Mini-mental state examination in Brazil. Arq Neuropsiquiatr. 2003; $61: 777-81$.

Brucki SMD. Epidemiology of Mild Cognitive Impairment in Brazil. Dement Neuropsychol. 2013;7(4):363-6.

Burton CL, Strauss E, Bunce D, Hunter MA, Hultsch DF. Functional Abilities in Older Adults with Mild Cognitive Impairment. Gerontology. 2009a;55:570-81.

Burton CL, Strauss E, Hultsch DF, Hunter MA. The relationship between Everyday Problem Solving and Inconsistency in Reaction Time in Older Adults. Aging Neuropsychol Cogn. 2009b;16:607-32. 
Bussab WO, Moretin PA. Estatística Básica. 5 ed. São Paulo: Editora Saraiva; 2002.

Bustamante SEZ, Bottino CMC, Lopes MA, Azevedo D, Hototian SR, Litvoc J, Jacob Filho W. Instrumentos combinados na avaliação de demência em idosos. Arq. Neuropsiquiatr. 2003;61(3-A):601-6.

Cardoso I. Aspectos transculturais na adaptação de instrumentos de avaliação psicológica. Interaç̧ões. 2006;10:98-112.

Capucho PHFV, Brucki SMD. Judgment in Mild Cognitive Impairment and Alzheimer's disease. Dement Neuropsychol. 2011;5(4):297-302.

Canali F, Brucki SMD, Bueno OFA. Behavioural assessment of the dysexecutive syndrome (BADS) in healthy elders an Alzheimer's disease patients Premliminary study. Dement Neuropsychol. 2007;2:153-9.

Cerami C, Cappa SF. The behavioral variant of frontotemporal dementia: linking neuropathology to social cognition. Neurol Sci.2013;34(8):1267-4.

César KG. Estudo da prevalência de comprometimento cognitivo leve e demência na cidade de Tremembé, estado de São Paulo [tese]. São Paulo: Universidade de São Paulo; 2014.

Channon S, Crawford S. Problem-solving in real-life-type situations: The effects of anterior and posterior lesions on performance. Neuropsychologia. 1999;757-70.

Channon S. Frontal lobe dysfunction and everyday problem-solving: Social and nonsocial contributions. Acta Psychol. 2004;115:235-54.

Ciaramelli E, Muccioli M, Làdavas E, Pellegrino G. Selective deficit in personal moral judgment following damage to ventromedial prefrontal cortex. Scan. 2007;2:84-92. 
Collins K, Luszcz M, Lawson M, Keeves J. Everyday problem solving in elderly women: Contributions of residence, perceived control, and age. Gerontologist. 1997; 37(3):293302.

Cornelius SW, Caspi A. Everyday Problem Solving in Adulthood and Old Age. Psychol and Aging, 1987;2:144-53.

Couture SH, Penn DL, Roberts DL. The Functional Significance of Social Cognition in Schizophrenia: A Review. Schizophr Bull. 2006;32:44-63.

Davis M. A plea for judgment. Sci Eng Ethics. 2012; 18:789-808.

Delazer M, Sinz H, Zamarian L, Benke T. Decision-making with explicit and stable rules in milg Alzheimer's disease. Neuropsychologia. 2007;45:1632-41.

Denney NW \& Pearce KA. A developmental study of practical problem solving in adults. Psychol Aging. 1989;4:438-42.

Drane DL, Osato SS. Using the Neurobehavioral Cognitive Status Examination as a screening measure for older adults. Arch Clin Neuropsychol. 1997;12:139-43.

Duke LM, Kaszniak, AW. Executive Control Functions in Degenerative Dementias: A Comparative Review. Neuropsychol Rev. 2000;10(2):75-99.

Espinosa A, Alegret M, Boada M, Vinyes G, Valero S, Martínez-Lage P et al. Ecological Assessment of executive functions in mild cognitive impairment and Alzheimer's disease. J Int Neuropsychol Soc. 2009;15:751-7.

Eslinger PJ, Moore P, Antani S, Anderson C, Grossman M. Apathy in frontotemporal dementia: behavioral and neuroimaging correlations. Behav Neurol.2012;25(2):127-36.

Etchells E, Darzins P, Silberfeld M, Singer PA, McKenny J, Naglie G et al. Assessment of patient capacity to consent to treatment. J Gen Intern Med. 1999;14:27-34. 
Evans SBT. Dual-Processing Accounts of Reasoning, Judgment and Social Cognition. Annu Rev Psychol. 2008; 59: 255-78.

Farias ST, Mungas D, Reed BR, Harvey D, Cahn-Weiner D, DeCarli C. MCI is associated with deficits in everyday functioning. Alzheimer Dis Assoc Disord. 2006;20(4):217-23.

Ferri CP, Prince M, Brayne C, Brodaty H, Fratiglioni L, Ganguli M, et al. Global prevalence of dementia: a Delphi consensus study. Lancet. 2005;366(9503):2112-7.

Fletcher RH, Fletcher SW. Epidemiologia Clínica. 4 ed. São Paulo: Artmed; 2006. Cap. 3, p. 56-81: Diagnóstico.

Folstein MF, Folstein SE, McHugh PR. Mini-mental state. A practical method for grading the cognitive state of patients for the clinician. J. Psychiatr Res.1975;12:189-98.

Fouty HE, Mullen CM, Weitzner DS, Mulcahy DJ. Correcting for Gender on the Cognistat Judgment Subtest. Appl Neuropsychol Adult. 2013;20:152-4.

Frith CD. Social Cognition. Philos Trans R Soc B. 2008;363:2033-39.

Frota NAF, Nitrini R, Damasceno BP, Forlenza OV, Dias-Tosta E, Silva AB et al. Criteria for the diagnosis of Alzheimer's disease. Recomendations of the Scientific Department of Cognitive Neurology and Agyng of the Brazilian Academy of Neurology. Dement Neuropsychol. 2011;5(3):146-52.

Gavett B E, Lou KR, Daneshvar DH, Green RC, Jefferson AL, Stern RA. Diagnostic Accuracy Statistics for Seven Neuropsychological Assessment Battery (NAB) Test Variables in the Diagnosis of Alzheimer's Disease Appl Neuropsychol Adult. 2012; 19(2):1-14. 
Gleichgerrcht E, Torralva T, Roca M, Manes F. Utility of an abbreviated version of the executive and social cognition battery in the detection of executive deficits in early behavioral variant frontotemporal dementia patients. Journal of the International Neuropsychological Society. 2010;16:687-94.

Gleichgerrcht E, Torralva T, Roca M, Pose M, Manes F. The role of social cognition in moral judgment in frontotemporal dementia. Soc Neurosci. 2011; 6(2): 113-22.

Godinho C, Camozzato AL, Onyszko D, Chaves ML. Estimation of the risk of conversion of mild cognitive impairment of Alzheimer type to Alzheimer's disease in a south Brazilian population-based elderly cohort: the PALA study. Int Psychogeriatr. 2012;24:674-81.

Graham JE, Rockwood K, Beattie BL, et al. Prevalence and severity of cognitive impairment with and without dementia in an elderly population. Lancet. 1997;349:793-6.

Greene JD, Sommerville RB, Nystrom LE, Darley JM, Cohen JD. An fMRI investigation of emotional engagement in moral judgment. Science. 2001;293:2105-8.

Grisso T, Appelbaum P, Hil-Fotouhi C. The MacCAT-T: a clinical tool to assess patient's capacities to make treatment decisions. Psychiatr Serv. 1997; 48: 1415-9.

Guillemin F, Bombardier C, Beaton D. Cross-cultural adaptation of health related quality of life measures: literature review and proposed guidelines. $J$ Clin Epidemiol.1993;46(12):1417-32.

Hair JF, Tatham RL, Anderson RE, Black W. Multivariate Data Analysis. 5 ed. Pearson Education; 2005. 
Harciarik M, Kertesz, A. Primary progressive aphasias and their contribution to the contemporary knowledge about the brain-language relationship. Neuropsychol Rev. 2011;21:271-287.

Hastie R. Problems for judgment and decision making. Annu Rev Psychol. 2001; 52: 65383.

Herrera E, Caramelli P, Silveira ASB, Nitrini R. Epidemiologic survey of dementia in a community-dwelling Brazilian population. Alzheimer Dis Assoc Disord. 2002;16(2):1038.

Hodges JR, Miller B. The classification, genetics and neuropathology of frontotemporal dementia. Introduction for the special topic papers: Part I. Neurocase. 2001;7(1):31-5.

Jack CR, Albert MS, Knopman DS, McKhann GM, Sperling RA, Camillo MC, et al. Introduction to the recommendations from the National Institute on Aging-Alzheimer's Association workgroups on diagnostic guidelines for Alzheimer's disease. Alzheimers Dement 2011;7(3):257-62.

Janofsky J, McCarthy R, Folstein M. The Hopkins Competency Assessment Test: a brief method for evaluationg patient's capacity to give informed consent. Hosp Community Psychiatry. 1992;42:132-6.

Jefferson AL, Byerly LK, Vanderhill S, Lambe S, Wong S, Ozonoff A, et al. Characterization of activities of daily living in individuals with mild cognitive impairment. Am J Geriatr Psychiatry. 2008;16(5):375-83.

Josephs KA, Whitwell JL, Weigand SD, Senjem ML, Boeve BF. Predicting functional decline in behavioral variant frontotemporal dementia. Brain.2011;134(Pt2):432-48.

Jorm, AF. A short form of the Informant Questionnaire on Cognitive Decline in the Elderly (IQCODE): development and cross-validation. Psychol Med. 1994;24:145-53. 
Kajanne, A. Structure and content: The relationship between reflective judgment and laypeople's viewpoints. $J$ Adult Dev. 2003;10:173-89.

Karlawish JHT, Casarett DJ, Bioethics JM, Xie SX, Kim SYH. The ability of persons with Alzheimer disease (AD) to make a decision about taking an $\mathrm{AD}$ treatment. Neurology. 2005;64:1514-9.

Kounti F, Tsolaki M, Kiosseoglou G. Functional cognitive assessment scale (FUCAS): a new scale to assess executive cognitive function in daily life activities in patients with dementia and mild cognitive impairment. Hum Psychopharmacol. 2006;21:305-11.

Lai JM, Karlawish J. Assessing the capacity to make everyday decisions: A guide for clinicians and an agenda for future research. Am J Geriatr Psychiatry. 2007;15:101-11.

Lezak MD, Howieson DB, Loring DW. Neuropsychological Assessment. 4th ed. New York: Oxford University Press; 2004.

Lim T, Marin DB. The assessment of decisional capacity. Neurol Clin. 2011;115-26.

Lima-Silva TB, Bahia VC, Nitrini R, Yassuda MS. Functional status in behavioral variant frontotemporal dementia: a systematic review. Biomed Res Int. 2013;1-7.

Liu LL, Gonzalez R. Judgment and decision process in older adults' compliance with medical regimens. Medical adherence and aging; social and cognitive perspectives. 2007; 201-32.

Loeb, PA. Independent Living Scales. Manual. San Antonio TX: Psychological Corporation; 1996.

Lopes MA, Bottino CM. Prevalência de demência em diversas regiões do mundo: Análise dos estudos epidemiológicos de 1994 a 2000. Arq Neuropsiquiatr.2002; 60(1):61-9. 
Lopes MA, Ferrioli E, Nakano EY, Litvoc J, Bottino CMC. High prevalence of dementia in a community-based survey of older people from Brazil: association with intellectual activity rather than education. J Alzheimers Dis. 2012;32(2):307-16.

MacDougall EE, Mansbach WE. The Judgment Test of the Neuropsychological Assessment Battery (NAB): Psychometric Considerations in an Assisted-Living Sample. Clin Neuropsychol. 2013;27(5):827-39.

Maioli F, Coveri M, Pagni P, Chiandetti C, Marchetti C, Ciarrocchi R et al. Conversion of Mild Cognitive Impairment to Dementia in Elderly subjects: A preliminary study in a memory and cognitive disorder unit. Arch. Gerontol. Geriatr. 2007;Suppl.1:233-41.

Malloy-Diniz LF, Lasmar VAP, Gazinelli LSR, Fuentes D, Salgado JV. Teste de aprendizagem auditivo-verbal de Rey: aplicabilidade na população idosa brasileira. Rev Bras Psiquiatr. 2007;29(4):324-9.

Mansbach WE, MacDougall EE, Clark KM, Mace RA. Preliminary investigation of the Kitchen Picture Test (KPT): A new screening test of practical judgment for older adults. Aging Neuropsychol Cogn. 2013:1-19.

Margrett JA, Allaire JC, Johnson TL, Daugherty KE, Weatherbee SR. Everyday Problem Solving. Aging in America. 2010;79-101.

Marson D, Ingram K, Cody H, Harrell LE. Assessing the competency of patients with Alzheimer's disease under different legal standards. Arch Neurol. 1995; 52:949-54.

Mayo AM, Wallhagen M, Cooper BA, Mehta K, Ross L, Miller B. The relationship between functional status and judgment/problem solving among individuals with dementia. Int J Geriatr Psychiatry. 2013;28:514-521. 
McKhann G, Drachman D, Folstein M, Katzman R, Price D, Stadlan EM. Clinical diagnosis of Alzheimer's disease: report of the NINCDS-ADRDA Work Group under the auspices of Department of Health and Human Services Task Force on Alzheimer's Disease. Neurology. 1984;34:939-44.

Mendez MF, Anderson E, Shapira JS. An Investigation of Moral Judgment in Frontotemporal Dementia. Cog Behav Neurol. 2005;18:193-7.

Moll J, Zahn R, Oliveira-Souza R, Krueger F, Grafman J. Opinion: the neural basis of human moral cognition. Nat Rev Neurosci. 2005;6:799-809

Nascimento E. Adaptação, validação e normatização do WAIS-III para uma amostra brasileira. In Wechsler D. WAIS-III: manual para administração e avaliação. São Paulo: Casa do Psicolólogo; 2004.

Nelson HE. A modified card sorting test sensitive to frontal lobe defects. Cortex. 1976; 12 (4):313-24.

Nitrini R, Lefèvre BH, Mathias SC, Caramelli P, Carrilho PE, Sauaia N et al. Neuropsychological tests of simple application for diagnosing dementia. Arq Neuropsiquiatr. 1994;52:457-65.

Nitrini R, Caramelli P, Herrera EJr, Porto CS, Charchat-Fichman H, Carthery MT et al. Performance of illiterate and literate nondemented elderly subjects in two tests of lingterm memory. J Int Neuropsychol Soc. 2004; 10:634-8.

Nitrini R, Caramelli P, Porto CS, Charchat-Fichman H, Formigoni AP, Carthery-Goulart MT et al. Brief Cognitive battery in the diagnosis of Mild Alzheimer's disease in subjects with medium and high levels of education. Dement Neuropsychol. 2007;1:32-6.

Nitrini R, Bottino CMC, Albala C, Capunay NSC, Ketzoian C, Rodriguez JJL et al. Prevalence of dementia in Latin America: a collaborative study of population-based cohorts. Int Psychogeriatr. 2009;21(4):622-30. 
Northern California Neurobehavioral Group, Inc. Manual for the Neurobehavioral Cognitive Status Exam. Fairfax: CA; 1988.

Pasquali L. Validade dos Testes Psicológicos: Será Possível Reencontrar o Caminho? Psicologia: Teoria e Pesquisa. 2007;23:99-107.

Perneczky R, Pohl C, Sorg C. Impairment of activities of daily living requiring memory or complex reasoning as part of MCI syndrome. Inter J Geriatr Psychiatry. 2006;21: $158-62$.

Petersen RC, Smith GE, Waring SC, Ivnik RJ, Tangalos EG. Kokmen E. Mild Cognitive Impairment: Clinical Characterization and Outcome. Arch Neurol.1999;56:303-8.

Petersen RC. Mild cognitive impairment as a diagnostic entity. J Int Med.2004; 256:18394.

Pfeffer, RI, Kusosaki, TT, Harrah Jr, CH, Chance JM, Filos, S. Measurement of functional Activities in Older Adults in the Community. J Gerontol. 1982; 37(3):323-9.

Pickens S, Ostwald SK, Murphy-Pace K, Bergstrom N. Systematic review of current executive function measures in adults with and without cognitive impairments. Int $J$ Evid Based Health. 2010;8:110-25.

Piguet O, Hornberger M, Mioshi E, Hodges JR. Behavioural-variant frontotemporal dementia: diagnosis, clinical staging and management. Lancet Neurol .2011;10(2):16272.

Rabin LA, Borgos MJ, Saykin AJ, Root MD, Wishart HA, Flashman LA, Sprehn GC, Santulli RB. (Dartmouth Medical School). Judgment in Older Adults with AD, MCI, and Cognitive Complaints: Development and Preliminary Psychometric Evaluation of the Dartmouth-Rabin Judgment Questionnaire (DRJQ) (Presented at the 330th Annual Meeting of the International Neuropsychology Society; 2005; St. Louis, MO). 
Rabin LA, Borgos MJ, Saykin AJ, Wishart HA, Crane PK, Nutter-Upham KE et al. Judgment in older adults: Development and psychometric evaluation of the Test of Practical Judgment (TOP-J). J Clin Exp Neuropsychol. 2007;29:752-67.

Rabin LA, Borgos MJ, Saykin AJ. A Survey of Neuropsychologists' Practices and Perspectives Regarding the Assessment of Judgment Ability. Appl Neuropsychol. 2008; 15:264-73.

Rabin LA, Saykin AJ, West JD, Borgos MJ, Wishart HA, Nutter-Upham KE, Flashman LA, Santulli RB. Judgment in Older Adults with Normal Cognition, Cognitive Complaints, MCI, and Mild AD: Relation to Regional Frontal Gray Matter. Brain Imaging Behav, 2009;3:212-9.

Rabin L, Aronov A, Chi S, Fogel J, Charcape M, Borgos M, Saykin A. (Brooklyn College and The Graduate Center of City University New York - CUNY, Albert Einstein College of Medicine,). The Test of Practical Judgment (TOP-J): Updated Normative Data and Validation in a Demographically Diverse Group of Older Adults. (Presented at the Annual Meeting 410 of the International Neuropsychology Society -INS; 2013; Hawaii).

Rascovsky K, Hodges JR, Kipps CM, Johnson JK, Seeley WW, Mendez MF et al. Diagnostic Criteria for the Behavioral Variant of Frontotemporal Dementia (bvFTD): Current Limitations and Future Directions. Alzheimer Dis Assoc Disord. 2007;21:S14-8).

Rascovsky K, Hodges JR, Knopman D, Mendez MF, Kramer JH, Neuhaus J et al. Sensitivity of revised diagnostic criteria for the behavioural variant of frontopemporal dementia. Brain, 2011;134:2456-77.

Revheim N, Medalia A. The Independent Living Scales as a Measure of Functional Outcome for Schizophrenia. Psychiatr Serv. 2004;55:1052-4.

Rey A. Teste de cópia e de reprodução de memória de figuras geométricas complexas: Manual. São Paulo: Casa do Psicólogo; 1999. 
Rogalski E, Cobia D, Harison TM, Wieneke C, Weintraub S, Mesulam M. Progression of language decline and cortical atrophy in subtypes of primary progressive aphasia. Neurology. 2011;76(24):1804-10.

Scazufca M, Menezes PR, Vallada HP, Crepaldi AL, Pastor-Valero M, Coutinho LMS, et al. High prevalence of dementia among older adults from poor socioeconomic backgrounds in São Paulo, Brazil. Int Psychogeriatr. 2008;20(2):394-405.

Shany-U R, Rankin KP. Personality and social cognition in neurodegenerative disease. Curr Opin Neurol. 2011;24:550-5.

Sinz H, Zamarian L, Benke T, Wenning GK, Delazer M. Impact of ambiguity and risk on decision making in mild Alzheimer's disease. Neuropsychologia. 2008;46:2043-55.

Spreen O, Strauss E, Sherman EMS. A Compendium of Neuropsychological Tests. Administration, Norms, and Commentary. 3rd ed. New York: Oxford University Press; 2006.

Stern RA; White T. Neuropsychological Assessment Battery: Administration, scoring, and interpretation manual. Lutz, FL: Psychological Assessment Resources; 2003.

Sunderland T, Hill JL, Mellow AM, Lawlor BA, Gundersheimer J, Newhouse PA et al. Clock Drawing in Alzheimer's disease: a novel measure of dementia severity. J Am Geriatr Soc. 1989;37:725-9.

Torralva T, Kipps CM, Hodges JR, Clark L, Bekinschtein T, Roca M et al. The relationship between affective decision-making and theory of mind in the frontal variant of fronto-temporal dementia. Neuropsychologia. 2007;45(2):342-9.

Torralva T, Roca M, Gleichgerrcht E, Bekinschtein T, Manes F. A Neuropsychological battery to detect specific executive and social cognitive impairments in early frontotemporal dementia. Brain. 2009;132:1299-309. 
Urbina S. Fundamentos de Testagem Psicológica. Porto Alegre: Artmed; 2007.

Wadley VG, Crowe M, Marsiske $M$ et al. Changes in Everyday Function in Individuals with Psychometrically Defined Mild Cognitive Impairment in the Advanced Cognitive Training for Independent and Vital Elderly Study. J Am Geriatr Soc. 2007;55:1192-8.

Ward A, Arrighi HM, Michels S, Cedarbaum JM. Mild Cognitive impairment: disparity of incidence and prevalence estimates. Alzheimers Dement. 2012;8:14-21.

Wechsler D. Manual for the Wechsler Adult Intelligence Scale-Revised. New York: The Psychological Corporation; 1981.

Wechsler D. Wechsler Memory Scale Manual. The Psychological Corporation Harcourt Brace Jovanovich; 1987.

Wechsler D. Wechsler Adult Intelligence Scale. 3rd ed. San Antonio, TX: The Psychological Corporation; 1997.

Willis SL, Allen-Burge R, Dolan MM, Bertrand RM, Yesavage J \& Taylor JL. Everyday problem solving among individuals with Alzheimer's disease. Gerontologist. 1998;38:569-77.

Wilson BA, Alderman N, Burguess PW, Emslie H, Evans JJ. Behavioural Assessment of the Dysexecutive Syndrome (BADS). Bury St Edmunds, UK: Thames Valley Test Company; 1996. Tradução: Souza RO, Schmidt SL. Rio de Janeiro: Cognição.

Winblad B, Palmer K, Kivipelto M, Jelic V, Fratiglioni L, Wahlund LO et al. Mild cognitive impairment - beyond controversies, towards a consensus: report of the International Working Group on Mild Cognitive Impairment. J Int Med. 2004; 256:240-6. 
Woods DC, Patterson MB, Whitehouse PJ. Utility of the Judgment Questionnaire Subtest of the Neurobehavioral Cognitive Status Examination in the Evaluation of Individuals with Alzheimer's Disease. Clin Gerontol. 2000;21(4):49-65.

World Health Organization (on-line). Dementia: a public health priority. (citado em 2012). Disponível em: http://www.who.int.

Yassuda MS, Nunes PV. Innovative psychosocial approaches in old age psychiatry. Curr Opin Psychiatry. 2009;22:527-31.

Yesavage JA, Brink TL, Rose TL, Lum O, Huang V, Adey M, Leirer VO. Development and validation of a geriatric depression scale: a preliminary report. $J$ Psichiat Res. 1983;17:37-49.

Zamarian L, Weiss EM, Delazer M. The impact of mild cognitive impairment on decision making in two gambling tasks. J Gerontol Psychol Sci. 2011;66:23-31.

Zgaljardic DJ, Yancy S, Temple RO, Watford MF, Miller R. Ecological validity of the Screening Module and the Daily Living Tests of the Neuropsychological Assessment Battery using the Mayo-Portland Adaptability Inventory-4 in post acute brain injury rehabilitation. Rehabilitation Psychology. 2011;56:359-65. 